Supporting Information for:

\title{
Reactivity of (Pyridine-Diimine)Fe Alkyl Complexes with Carbon Dioxide
}

\author{
Ka-Cheong Lau, Richard F. Jordan* \\ Department of Chemistry, The University of Chicago, \\ 5735 South Ellis Avenue, Chicago, Illinois 60637, United States \\ Email: rfjordan@uchicago.edu
}

\section{Contents}

I. Summary of X-ray Diffraction Data for 1-PMe 3 and 4

II. Solution Properties of Compounds

III. Additional Experimental Procedures

IV. IR Assignments

V. Temperature-Dependent SQUID Magnetization Data

$\begin{array}{lll}\text { VI. } & \text { EPR Spectrum of 1-PMe } & \text { S22 }\end{array}$

VII. NMR Spectra for 1-PMe 3 , 2-THF, 3-PMe 3 , 4, and 4-THF S23

VIII. Crystallographic Report of 1-PMe 3

IX. Crystallographic Report of $4 \quad$ S54 


\section{Summary of X-ray Diffraction Data for 1-PMe 3 and 4}

Table S-1. Summary of X-ray Diffraction Data for 1-PMe 3 and 4.

\begin{tabular}{|c|c|c|}
\hline & 1-PMe 3 & 4 \\
\hline Formula & $\mathrm{C}_{37} \mathrm{H}_{55} \mathrm{FeN}_{3} \mathrm{P}$ & $\mathrm{C}_{59} \mathrm{H}_{66} \mathrm{BFeN}_{3} \mathrm{O}_{2}$ \\
\hline Formula weight & 628.66 & 915.80 \\
\hline Crystal system & Monoclinic & Monoclinic \\
\hline Space group & $P 2_{1} / c$ & $P 2_{1} / c$ \\
\hline$a(\AA)$ & $17.2088(12)$ & $12.5740(8)$ \\
\hline$b(\AA)$ & $12.6102(8)$ & $12.6375(8)$ \\
\hline$c(\AA)$ & $16.3707(11)$ & $31.6380(19)$ \\
\hline$\beta(\operatorname{deg})$ & $100.567(2)$ & $96.473(2)$ \\
\hline $\mathrm{V}\left(\AA^{3}\right)$ & $3492.3(4)$ & $4995.4(5)$ \\
\hline$Z$ & 4 & 4 \\
\hline$T(\mathrm{~K})$ & 100 & 100 \\
\hline Crystal color, habit & green, block & red, brick \\
\hline GOF on $F^{2}$ & 1.045 & 1.073 \\
\hline $\begin{array}{l}\text { R indices } \\
(I>2 \sigma(I))^{a}\end{array}$ & $\mathrm{R}_{1}=0.0751, \mathrm{wR}_{2}=0.1483$ & $\mathrm{R} 1=0.0530, \mathrm{wR} 2=0.0995$ \\
\hline $\begin{array}{l}\text { R indices } \\
\text { (all data) }{ }^{a}\end{array}$ & $\mathrm{R}_{1}=0.0933, \mathrm{wR}_{2}=0.1560$ & $\mathrm{R} 1=0.0847, \mathrm{wR} 2=0.1084$ \\
\hline \multicolumn{3}{|c|}{$\begin{array}{l}{ }^{a} \mathrm{R} 1=\Sigma|| F_{\mathrm{o}}|-| F_{\mathrm{c}} \| / \Sigma\left|F_{\mathrm{o}}\right| ; \mathrm{wR} 2=\left[\Sigma\left[w\left(F_{\mathrm{o}}^{2}-\mathrm{F}_{\mathrm{c}}^{2}\right)^{2}\right] / \Sigma\left[w\left(F_{\mathrm{o}}^{2}\right)^{2}\right]\right]^{1 / 2}, \text { where } w=q\left[\sigma^{2}\left(F_{\mathrm{o}}^{2}\right)+(a P)^{2}+\right. \\
b P]^{-1}\end{array}$} \\
\hline
\end{tabular}




\section{Solution Properties of Compounds}

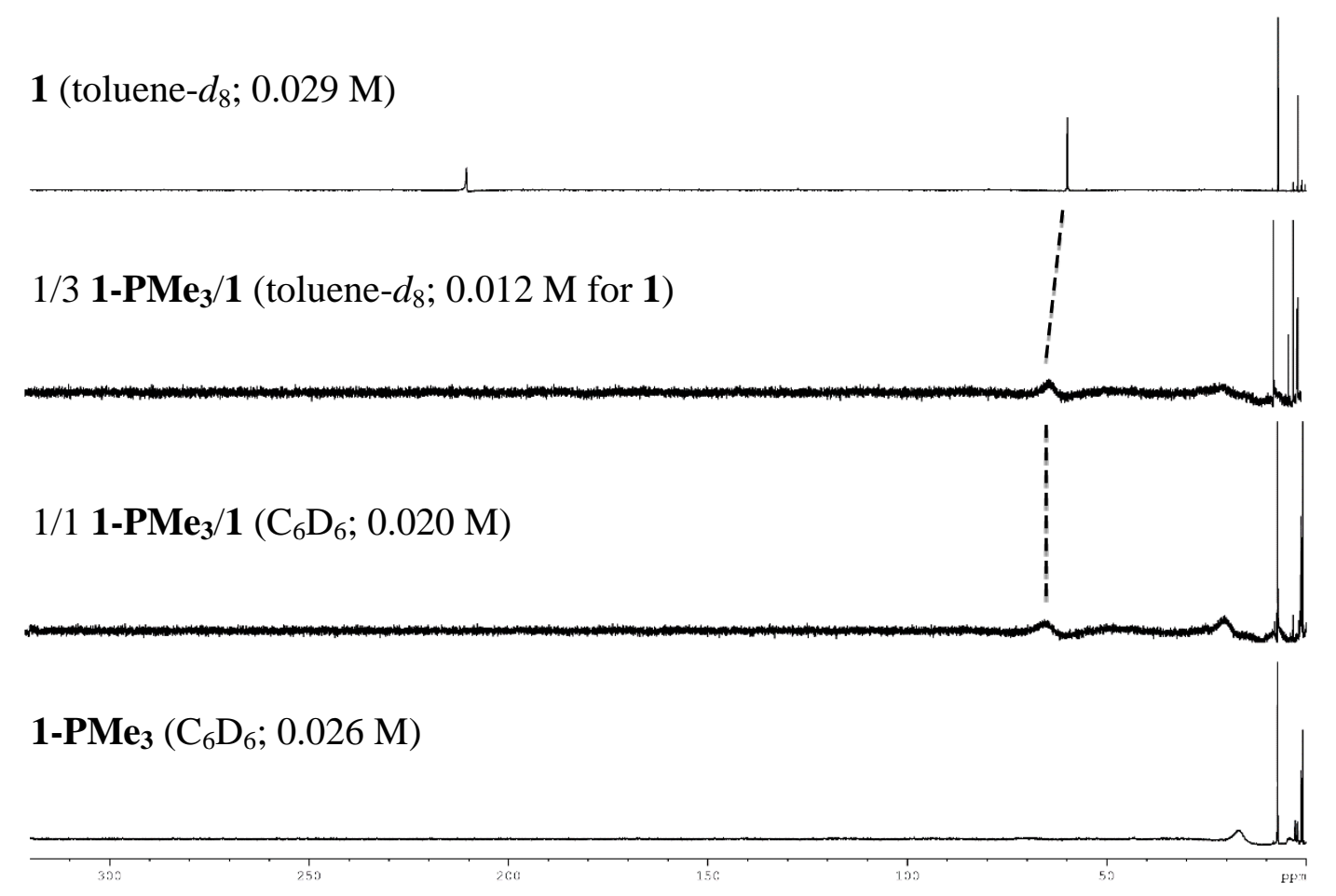

Figure S-1. ${ }^{1} \mathrm{H}$ NMR spectra of 1, 1-PMe 3 and mixtures of $\mathbf{1 - P M e _ { 3 }}$ and $\mathbf{1}$ at $23{ }^{\circ} \mathrm{C}(\delta 320-0)$.

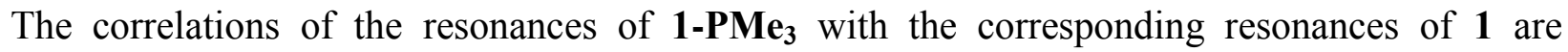
indicated by dashed lines. The resonance for the $\mathrm{Fe}-\mathrm{PMe}$ group of $\mathbf{1 - P M e _ { 3 }}$ appears at $\delta 17$. No

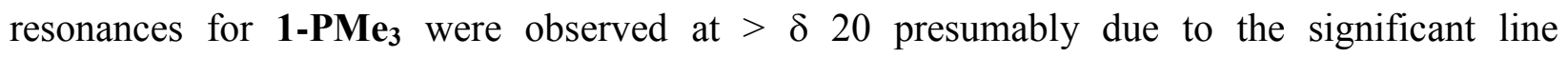
broadening. 


\section{2-THF $\left(\right.$ THF- $d_{8}$ )}

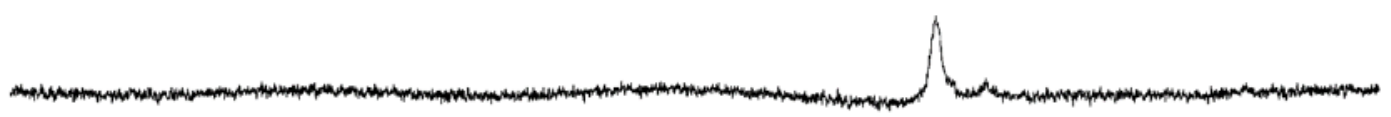

\section{2-THF $\left(\mathrm{C}_{6} \mathrm{D}_{5} \mathrm{~F}\right)$}

$2\left(\mathrm{C}_{6} \mathrm{D}_{5} \mathrm{~F}\right)$

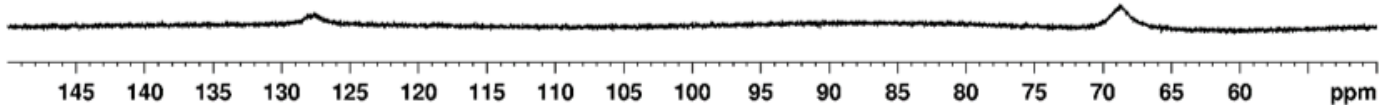

Figure S-2a. ${ }^{1} \mathrm{H}$ NMR spectra of 2 and 2-THF at $23{ }^{\circ} \mathrm{C}(\delta 150-50)$.
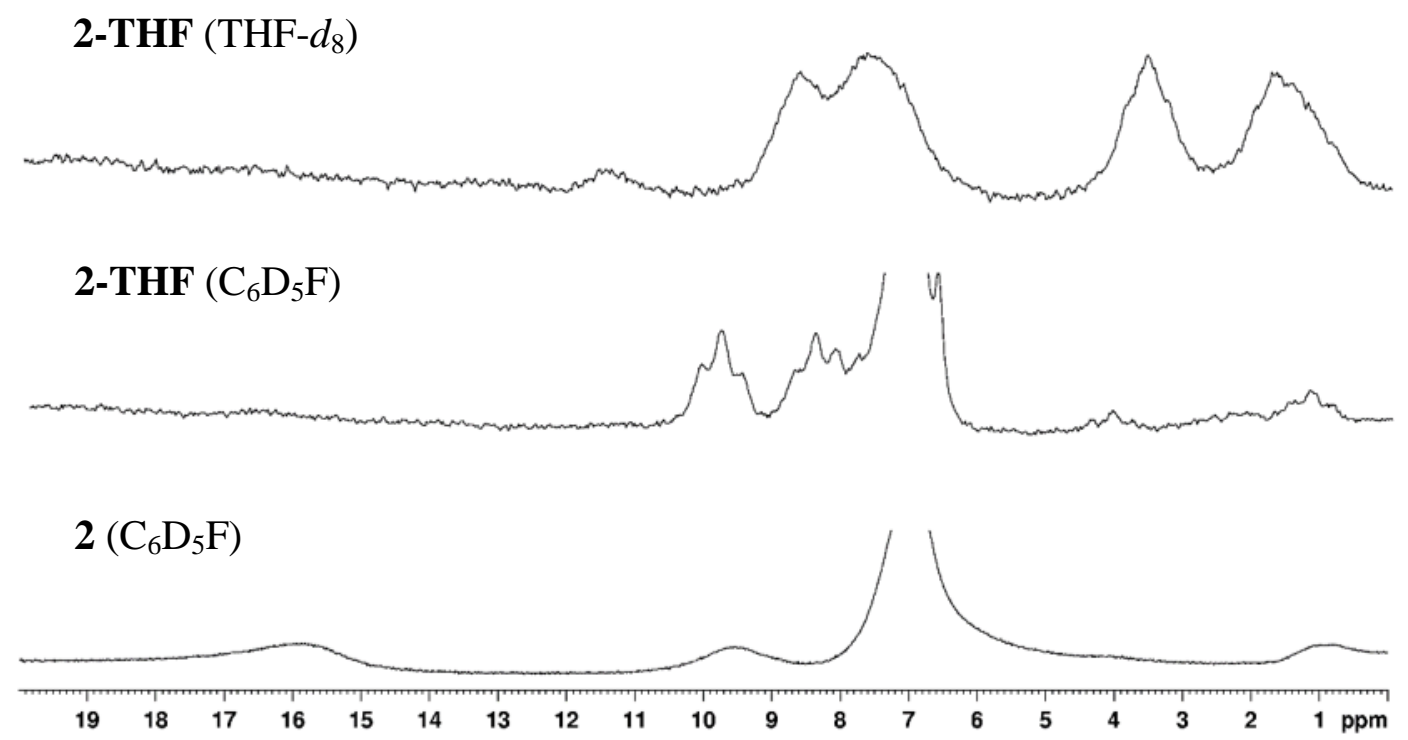

Figure S-2b. ${ }^{1} \mathrm{H}$ NMR spectra of 2 and 2-THF at $23{ }^{\circ} \mathrm{C}(\delta 20-0)$. 

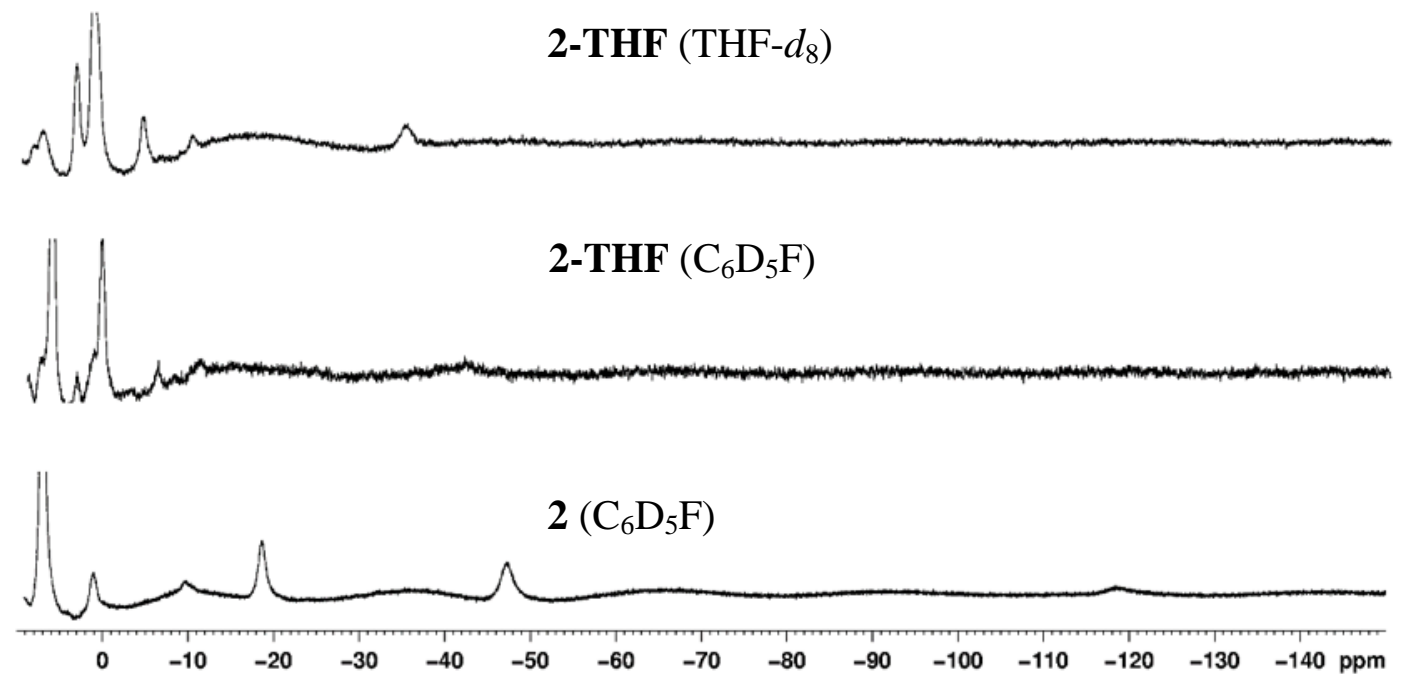

Figure S-2c. ${ }^{1} \mathrm{H}$ NMR spectra of $\mathbf{2}$ and $\mathbf{2 - T H F}$ at $23{ }^{\circ} \mathrm{C}(\delta 10--150)$. 


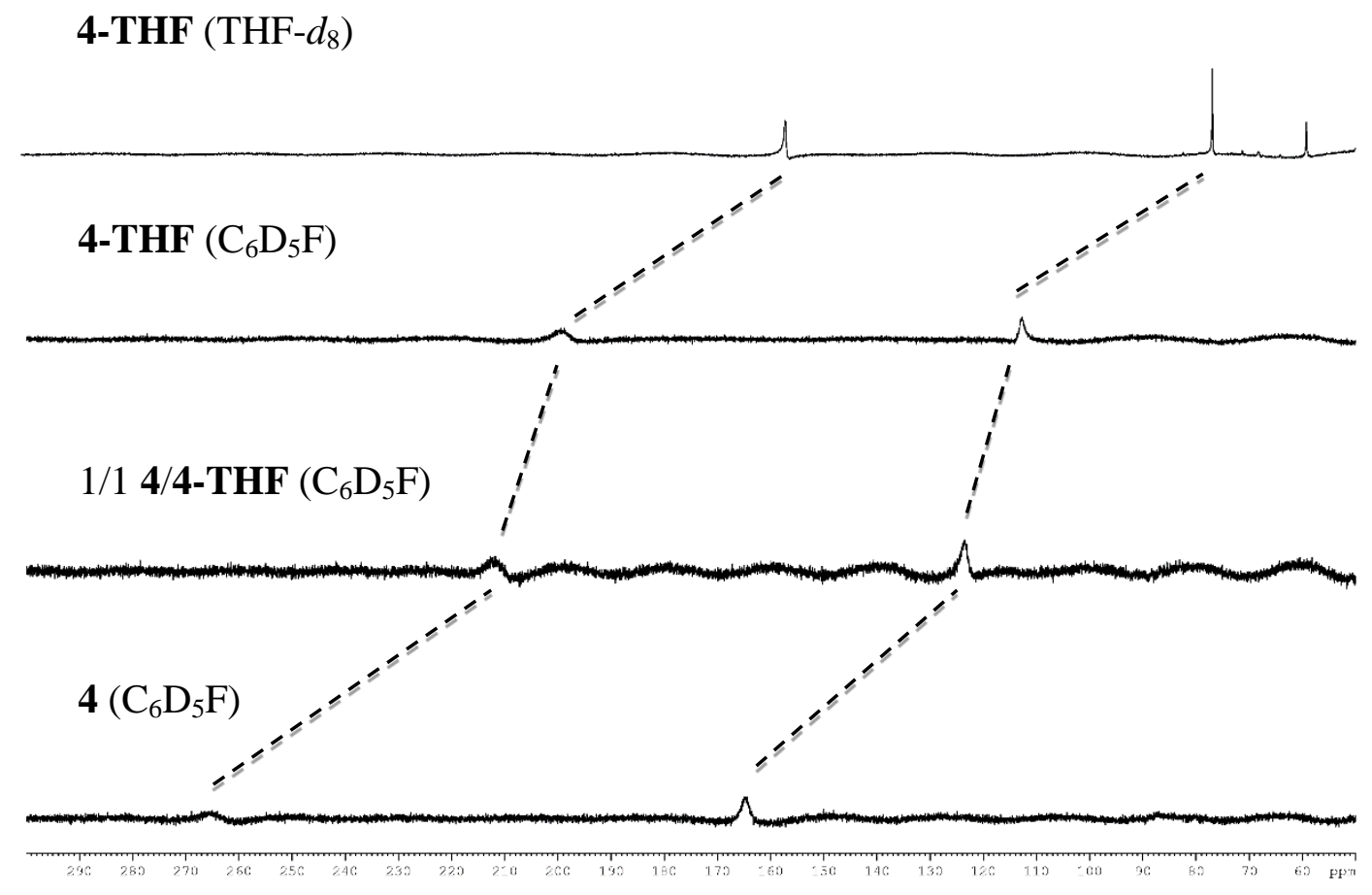

Figure S-3a. ${ }^{1} \mathrm{H}$ NMR spectra of 4, 4-THF and a mixture of 4/4-THF, at $23^{\circ} \mathrm{C}(\delta 300-50)$. The correlations of the resonances of $\mathbf{4}$ and $\mathbf{4 - T H F}$ are indicated by dashed lines. 


\section{4-THF $\left(\right.$ THF- $\left.d_{8}\right)$}

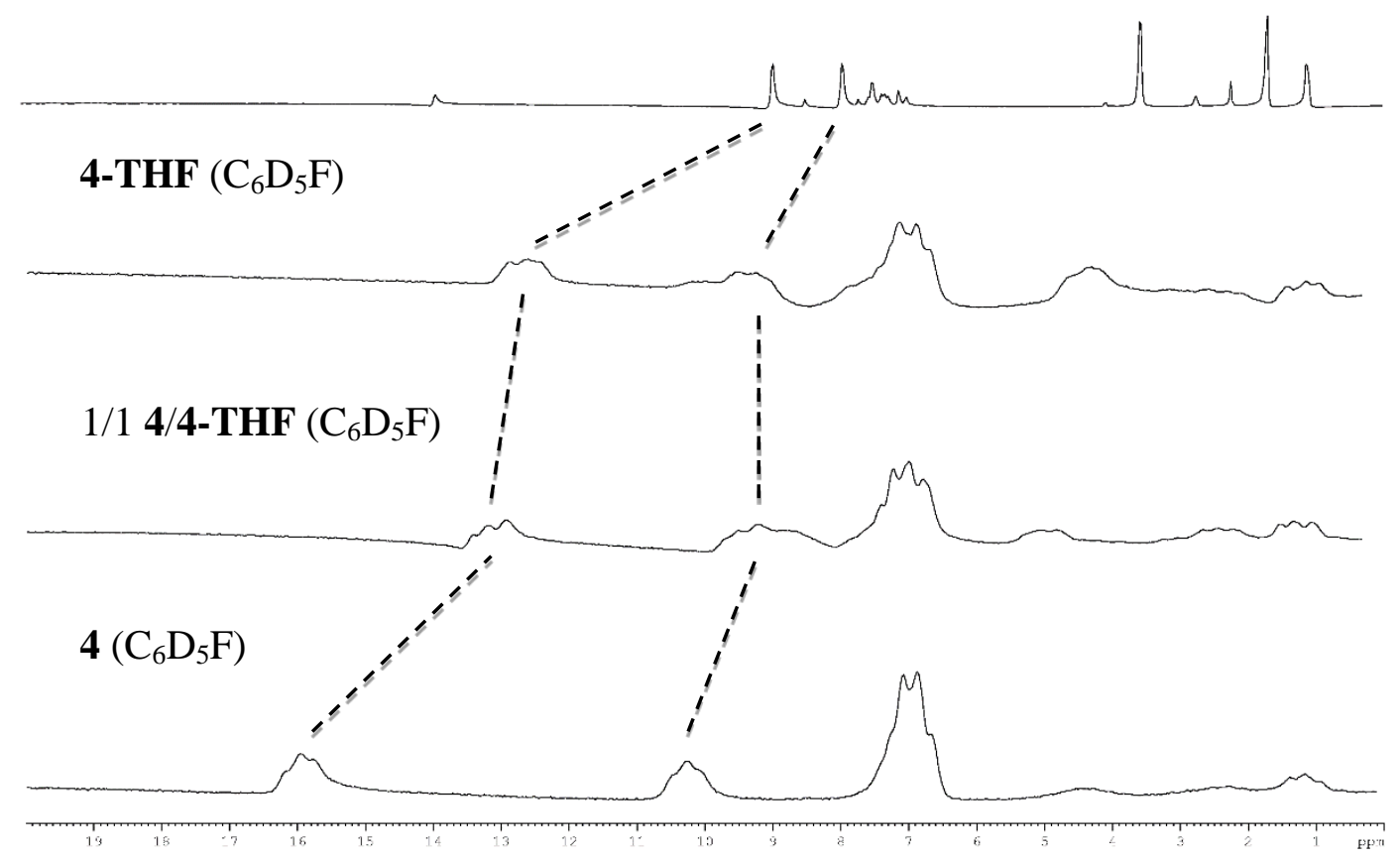

Figure S-3b. ${ }^{1} \mathrm{H}$ NMR spectra of $\mathbf{4}, \mathbf{4 - T H F}$ and a mixture of $\mathbf{4} / \mathbf{4}$-THF, at $23{ }^{\circ} \mathrm{C}(\delta 20-0)$. The correlations of the resonances of $\mathbf{4}$ and $\mathbf{4 - T H F}$ are indicated by dashed lines. 


\section{4-THF (THF- $\left.d_{8}\right)$}

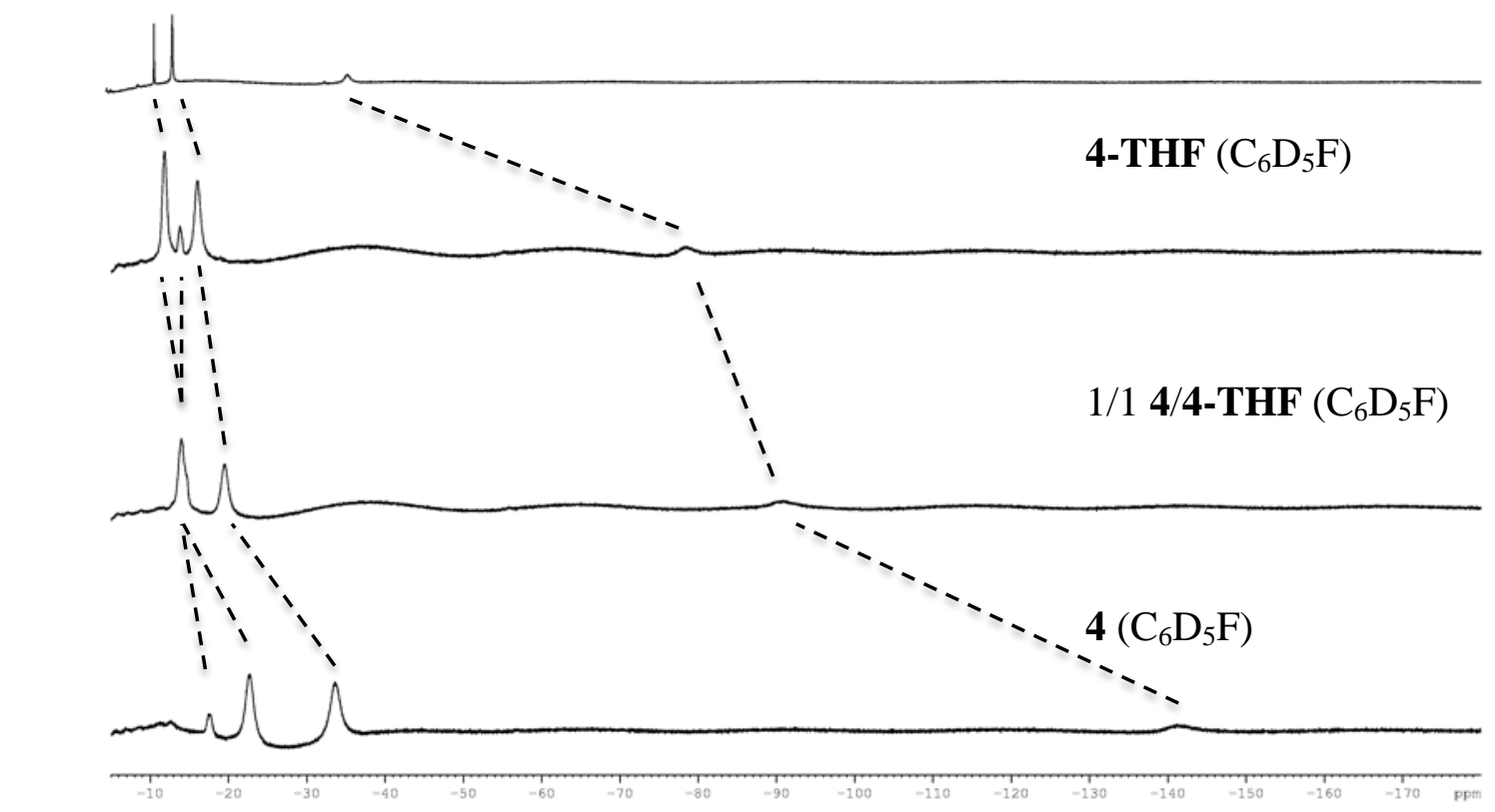

Figure S-3c. ${ }^{1} \mathrm{H}$ NMR spectra of 4, 4-THF and a mixture of 4/4-THF, at $23{ }^{\circ} \mathrm{C}(\delta-5--180)$. The correlations of the resonances of 4 and 4-THF are indicated by dashed lines.

\section{Additional Experimental Procedures}

Determination of Dissociation Equilibrium Constant for 1-PMe 3 by ${ }^{1} \mathrm{H}$ NMR. The equilibrium constant $\left(K_{\mathrm{eq}}\right)$ for dissociation of $\mathrm{PMe}_{3}$ from 1-PMe $\left(\mathrm{Scheme}_{3}\right)$ is defined by eq 1:

$$
K_{e q}=\frac{[1]\left[\mathrm{PMe}_{3}\right]}{\left[1-\mathrm{PMe}_{3}\right]}
$$

The observed chemical shifts of 1-PMe $\mathbf{e}_{3}$ in $\mathrm{C}_{6} \mathrm{D}_{6}$ are mole-fraction-weighted averages of the chemical shifts of $\mathbf{1}$ and $\mathbf{1 - P M \mathbf { P } _ { 3 }}$ and are given by eq 2 :

$$
\delta_{\text {obs }}=\chi_{1} \delta_{1}+\chi_{1-\mathrm{PMe} 3} \delta_{1-\mathrm{PMe} 3}
$$

where $\delta_{\text {obs }}=$ observed chemical shift; $\delta_{1}=$ chemical shift for $1 ; \delta_{1-P M e 3}=$ chemical shift for 1- 
$\mathbf{P M e}_{3} ; \chi_{1}$ and $\chi_{1-\mathrm{PMe} 3}$ are the mole fractions of $\mathbf{1}$ and $\mathbf{1 - P M e}$.

Combining eq 1 and 2 ,

$$
\begin{aligned}
{[\mathrm{Fe}]_{\text {total }} \delta_{\text {obs }} } & =[\mathbf{1}] \delta_{\mathbf{1}}+\left[\mathbf{1}-\mathbf{P M e} \mathbf{P M}_{3}\right] \delta_{\mathbf{1}-\mathbf{P M e} 3} \\
& =\left([\mathrm{Fe}]_{\text {total }}-\left[\mathbf{1}-\mathbf{P M e}_{3}\right]\right) \delta_{1}+\left[\mathbf{1}-\mathbf{P M e} \mathbf{e}_{3}\right] \delta_{\mathbf{1 - P M e}}
\end{aligned}
$$

where $[\mathrm{Fe}]_{\text {total }}=[\mathbf{1}]+\left[\mathbf{1}-\mathbf{P M e}_{3}\right]$

Rearranging,

$$
[\mathrm{Fe}]_{\text {total }}\left(\delta_{\mathrm{obs}}-\delta_{1}\right)=[\mathbf{1 - P M e}]\left(\delta_{1-\mathrm{PMe} 3}-\delta_{1}\right)
$$

Defining $\Delta \delta_{\text {obs }}=\delta_{\text {obs }}-\delta_{1}$

and $\Delta \delta_{1-\mathrm{PMe} 3}=\delta_{1-\mathrm{PMe} 3}-\delta_{1}$,

$$
[\mathbf{1 - P M e}]=\frac{[F e]_{t o t a l} \Delta \delta_{o b s}}{\Delta \delta_{1-P M e 3}}
$$

and

$$
\begin{aligned}
{[\mathbf{1}] } & =[\mathrm{Fe}]_{\text {total }}-\left[\mathbf{1}-\mathbf{P M e}_{3}\right] \\
& =\frac{[\mathrm{Fe}]_{\text {total }}\left(\Delta \delta_{1-P M e 3}-\Delta \delta_{o b s}\right)}{\Delta \delta_{1-P M e 3}}
\end{aligned}
$$

Therefore, without externally added $\mathrm{PMe}_{3}$, where $[\mathbf{1}]=\left[\mathrm{PMe}_{3}\right], K_{\mathrm{eq}}$ is given by eq $3 .^{5 \mathrm{a}}$

$$
K_{e q}=\frac{[F e]_{t o t a l}\left(\Delta \delta_{1-P M e_{3}}-\Delta \delta_{o b s}\right)^{2}}{\Delta \delta_{1-P M e_{3}} \Delta \delta_{o b s}}
$$

Eq 3 contains $K_{\text {eq }}$ and $\Delta \delta_{1-\mathrm{PMe} 3}$ as unknowns, and [Fe] $]_{\text {total }}$ and $\Delta \delta_{\text {obs }}$ as experimentally determined variables. $K_{\text {eq }}$ was determined using the graphical method described by Rose and Drago in ref $19 \mathrm{~b}$ of the text. To determine $K_{\text {eq }}$, a series of $\Delta \delta_{\text {obs }}$ values were measured for a series of $[\mathrm{Fe}]_{\text {total }}$ values. For each set of $\Delta \delta_{\text {obs }}$ and $[\mathrm{Fe}]_{\text {total }}$ values, $K_{\text {eq }}$ was calculated for a range of $\Delta \delta_{1}$. PMe3 values, and plots of $K_{\text {eq }}$ vs $\Delta \delta_{1-P M e 3}$ were generated according to eq 3. Since $K_{\text {eq }}$ and $\Delta \delta_{1-P M e 3}$ are independent of $[\mathrm{Fe}]_{\text {total }}$, the $K_{\text {eq }}$ vs $\Delta \delta_{1-P M e}$ curves simulated at different $[\mathrm{Fe}]_{\text {total }}$ (and corresponding $\left.\Delta \delta_{\text {obs }}\right)$ values should intersect at two points $\left(\Delta \delta_{1-P M e}, K_{\text {eq }}\right)$, corresponding to the 
two solutions of eq 3. As shown in Figure S-4, this result was indeed observed for 1-PMe . The two solutions of eq $3\left(\Delta \delta_{1-\mathrm{PMe} 3}, K_{\text {eq }}: 17.8(3), 1.3(2) \times 10^{-3} \mathrm{M} ; 21.5(6), 2.5(5) \times 10^{-3} \mathrm{M}\right)$ correspond to a $\delta_{1 \text {-PMe3 }}$ value that is $<\delta_{\text {obs }}$ and a $\delta_{1 \text {-PMe3 }}$ value that is $>\delta_{\text {obs }}$ respectively. However, as noted in the text, the observed ${ }^{1} \mathrm{H}$ resonances of $\mathbf{1 - P M \mathbf { P } _ { 3 }}$ shift toward those of $\mathbf{1}$ upon dilution, and away from those of 1 upon addition of $\mathrm{PMe}_{3}$. Therefore, $\delta_{1-\mathrm{PMe} 3}>\delta_{\mathrm{obs}}>\delta_{1}$, and the solution for which $\delta_{1 \text {-PMe3 }}<\delta_{\text {obs }}$ of eq $3\left(K_{\text {eq }}=2.5 \times 10^{-3} \mathrm{M}\right.$ at $\left.\Delta \delta_{1-\mathrm{PMe} 3}=21.5\right)$ is physically unreasonable and was rejected. The other solution provided values for $K_{\text {eq }}$ and $\Delta \delta_{1 \text { - }}$ PMe3. From plots of $K_{\text {eq }}$ vs $\Delta \delta_{1-P M e}$ for different resonances, it was determined that $K_{\text {eq }}\left(23^{\circ} \mathrm{C}\right)$ for $\mathbf{1 - P M e}=1.8(9) \times 10^{-3} \mathrm{M}$.

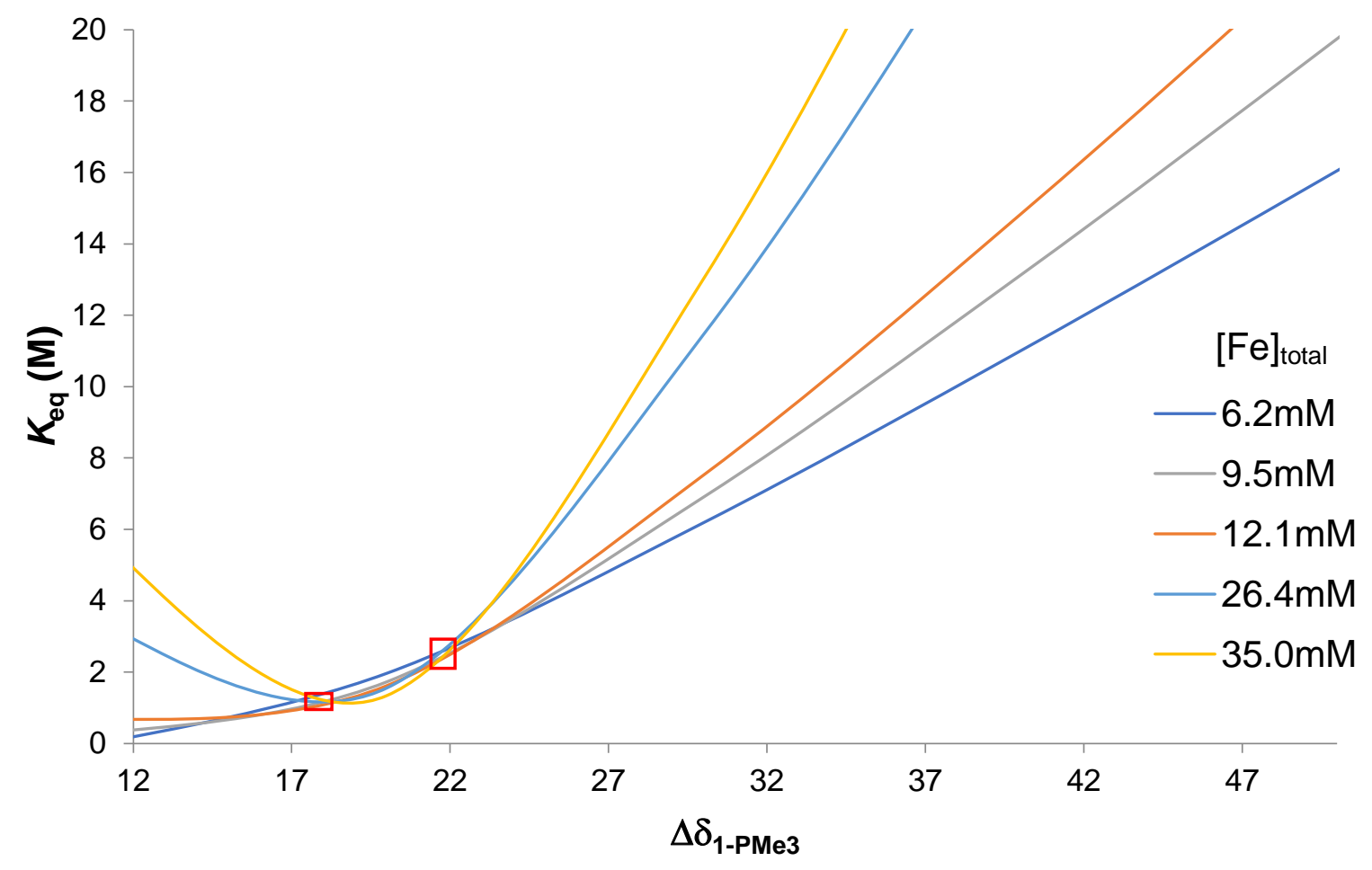

Figure S-4. Representative Rose-Drago plot of $K_{\text {eq }}$ vs $\Delta \delta_{1-\mathrm{PMe} 3}$ for the $\mathrm{CHMe} 2$ resonance ( $\delta-5.3$, $0.027 \mathrm{M}$ ) of a solution of $\mathbf{1 - P M e} \mathbf{P}_{3}$ in $\mathrm{C}_{6} \mathrm{D}_{6}$. The two solutions to eq 3 are the intersection points 
on this plot: $K_{\mathrm{eq}}=1.3(2) \times 10^{-3} \mathrm{M}, \Delta \delta_{1-\mathrm{PMe} 3}=17.8(3)$ and $K_{\mathrm{eq}}=2.5(5) \times 10^{-3} \mathrm{M}, \Delta \delta_{1-\mathrm{PMe} 3}=$ 21.5(6). Error bars are indicated by the red boxes. The latter solution was rejected as it gave a physically unreasonable $\delta_{1 \text {-PMe3 }}$ value as discussed above.

Kinetic Analysis of the Reaction of 1 with $\mathrm{CO}_{2}$. A valved J. Young NMR tube was charged with 1 ( $5.8 \mathrm{mg}, 0.010 \mathrm{mmol})$. Toluene- $d_{8}(0.5 \mathrm{~mL})$ was added by vacuum transfer at $-196{ }^{\circ} \mathrm{C}$. The mixture was thawed at $0{ }^{\circ} \mathrm{C}$ and exposed to $\mathrm{CO}_{2}(1 \mathrm{~atm}, 10$ equiv). The tube was then rapidly inserted into an NMR probe that had been pre-cooled at $0{ }^{\circ} \mathrm{C} .{ }^{1} \mathrm{H}$ NMR spectra were recorded periodically. Representative spectra are shown in Figure 4 in the text.

The rate equation for the conversion of $\mathbf{1}$ to 3 is given by eq 4 .

$$
\text { Rate }=k_{1}^{\prime}[\mathbf{1}]=k_{1}[\mathbf{1}] P_{\mathrm{CO} 2}
$$

where $k_{1}{ }^{\prime}=$ observed first-order rate constant; $k_{1}=$ second-order rate constant and $P_{\mathrm{CO} 2}=$ pressure of $\mathrm{CO}_{2}$.

The logarithmic form of first-order rate equation for the disappearance of $\mathbf{1}$ is given by eq 5 .

$$
\ln \left(I_{1}\right)=-k_{1}{ }^{\prime} t+\ln \left(I_{1,0}\right)
$$

where $I_{1}=$ integral value of the $\mathrm{CHMe} 2$ resonance of $\mathbf{1}$ at $\delta-11$ relative to the integral value of $\mathrm{Et}_{2} \mathrm{O}$ resonance $(\delta 1.2$, internal standard $)$ at time $=\mathrm{t} ; I_{\mathbf{1}, 0}=$ integral value of the $\mathrm{CHMe}$ resonance of 1 at $\delta-11$ relative to the integral value of $\mathrm{Et}_{2} \mathrm{O}$ resonance at the start of the reaction

First-order kinetic plots were generated using the program Origin 8 . The first-order kinetic plot for the disappearance of $\mathbf{1}$ is shown on the left of Figure 5 in the text. From this plot, $k_{1}^{\prime}\left(0^{\circ} \mathrm{C}\right)=3.63(9) \times 10^{-3} \mathrm{~s}^{-1}$.

The logarithmic form of first-order rate equation for the appearance of 3 is given by eq 6 . 


$$
\ln \left(I_{3, \infty}-I_{3}\right)=-k_{1} ’ \mathrm{t}+\ln \left(I_{3, \infty}\right)
$$

where $I_{3, \infty}=$ integral value of the $m$-Ar resonance of $\mathbf{3}$ relative to the integral value of the $\mathrm{Et}_{2} \mathrm{O}$ resonance $\left(\delta 1.2\right.$, internal standard) at the end of reaction. $I_{3}=$ integral value of the $m-\mathrm{Ar}$ resonance of 3 relative to the $\mathrm{Et}_{2} \mathrm{O}$ resonance at time $=\mathrm{t}$

The first-order plot for the appearance of $\mathbf{3}$ is shown Figure S-5. From this plot, $k_{1}{ }^{\prime}\left(0^{\circ} \mathrm{C}\right)$ $=3.5(2) \times 10^{-3} \mathrm{~s}^{-1}$, which is in good agreement with the $k_{1}$ ' value determined from the disappearance of $\mathbf{1}$.

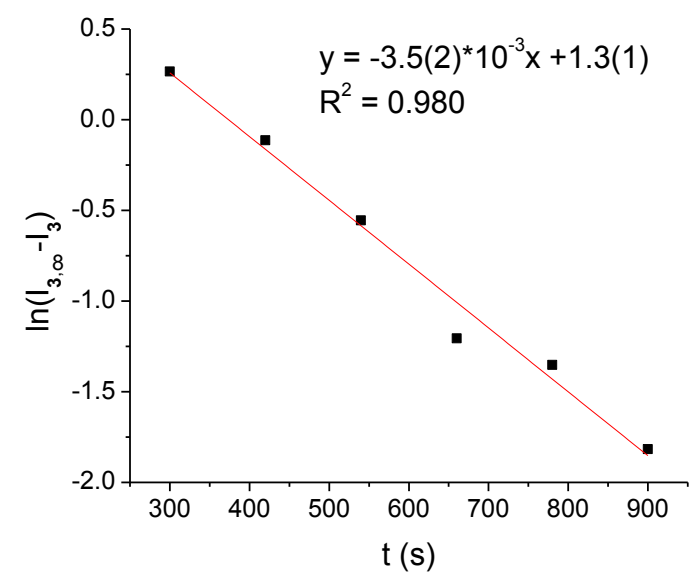

Figure S-5. Representative first-order kinetic plot for the reaction of 1 with $\mathrm{CO}_{2}(1 \mathrm{~atm})$ in toluene- $d_{8}\left(0^{\circ} \mathrm{C}\right)$. A plot for the appearance of $\mathbf{3}$ is shown $\left(k_{1}{ }^{\prime}=3.5(2) \times 10^{-3} \mathrm{~s}^{-1}\right)$.

The first-order rate constants for the conversion of 1 to 3 were obtained at $-25^{\circ} \mathrm{C}$ over the $P_{\mathrm{CO} 2}$ range of $0-6.1 \mathrm{~atm}$. A plot of $k_{1}$ ' vs. $P_{\mathrm{CO} 2}$ is shown on the right of Figure 5 in the text. The slope of this plot provides the second-order rate constant $k_{1}=6.2(2) \times 10^{-4} \mathrm{~atm}^{-1} \mathrm{~s}^{-1}$. 
The second-order rate constants for the conversion of $\mathbf{1}$ to $\mathbf{3}$ were obtained over the temperature range of $-35^{\circ} \mathrm{C}-0{ }^{\circ} \mathrm{C}$. The Eyring plot using these values is shown in Figure 6 in the text and provides activation parameters.

Kinetic Analysis of the Reaction of 1-PMe 3 with $\mathrm{CO}_{2}$. A valved J. Young NMR tube was charged with 1 (4.5 mg, $0.0081 \mathrm{mmol}), \mathrm{Cp}_{2} \mathrm{Fe}(0.8 \mathrm{mg}, 0.004 \mathrm{mmol}$, internal standard) and a solution of $\mathrm{PMe}_{3}$ in $\mathrm{C}_{6} \mathrm{D}_{6}(0.05 \mathrm{M}, 0.5 \mathrm{~mL}, 0.03 \mathrm{mmol}) .{ }^{1} \mathrm{H}$ NMR spectroscopy showed that an equilibrium mixture of $\mathbf{1 - P M \mathbf { P } _ { 3 }}$ and $\mathbf{1}$ had formed. The mixture was degassed and exposed to $\mathrm{CO}_{2}$ (1 atm, 13 equiv). The J. Young tube was then inserted into an NMR probe that was equilibrated at $23{ }^{\circ} \mathrm{C} .{ }^{1} \mathrm{H}$ NMR spectra were recorded periodically. Representative spectra are shown in Figure 7 in the text.

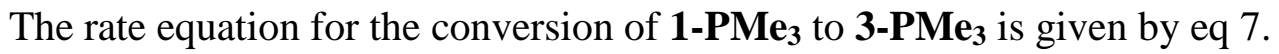

$$
\text { Rate }=k_{\text {obs }}[\mathrm{Fe}-\mathrm{Me}]=k_{\mathrm{obs}}\left(\left[\mathbf{1}-\mathbf{P M e}_{3}\right]+[\mathbf{1}]\right)
$$

where $k_{\mathrm{obs}}=$ observed first-order rate constant

and $[\mathrm{Fe}-\mathrm{Me}]=[\mathbf{1 - P M e}]+[\mathbf{1}]$

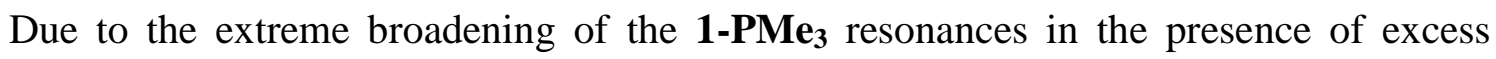
$\mathrm{PMe}_{3}$, the kinetics were evaluated by analyzing the appearance of $\mathbf{3}-\mathbf{P M e}_{3}$, by monitoring the appearance of the $\mathrm{CHMe} e_{2}$ resonance at $\delta-18$. The first-order rate equation for the appearance of 3-PMe $\mathbf{P M}_{3}$ (exponential form) is given by eq 8 .

$$
I_{\mathrm{Fe}}=-I_{\mathrm{Fe}, \infty} \exp \left(-k_{\mathrm{obs}} \mathrm{t}\right)+I_{\mathrm{Fe}, \infty}
$$

where $I_{\mathrm{Fe}}=$ integral value of the $\delta-18$ resonance of $\mathbf{3}-\mathbf{P M e}_{3}$ relative to the integral value of the $\mathrm{Cp}_{2} \mathrm{Fe}$ resonance $(\delta 4.1$, internal standard $)$ at time $=\mathrm{t} ; I_{\mathrm{Fe}, \infty}=$ integral value of the $\delta-18$ resonance of $3-\mathrm{PMe}_{3}$ relative to the integral value of the $\mathrm{Cp}_{2} \mathrm{Fe}$ resonance at the end of the reaction 
First-order kinetic plots were generated using the program Origin 8. A representative first-order plot is shown in Figure 8.

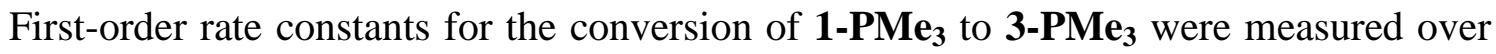
the $\mathrm{PMe}_{3}$ concentration range of $0.04 \mathrm{M}-0.17 \mathrm{M}$. Representative first-order kinetic plots are shown in Figure S-6. The conversion of $\mathbf{1 - P M e}$ to $\mathbf{3 - P M e} 3$ was found to be strongly inhibited by $\mathrm{PMe}_{3}$, and the observed first-order rate constants are tabulated in Table 2.
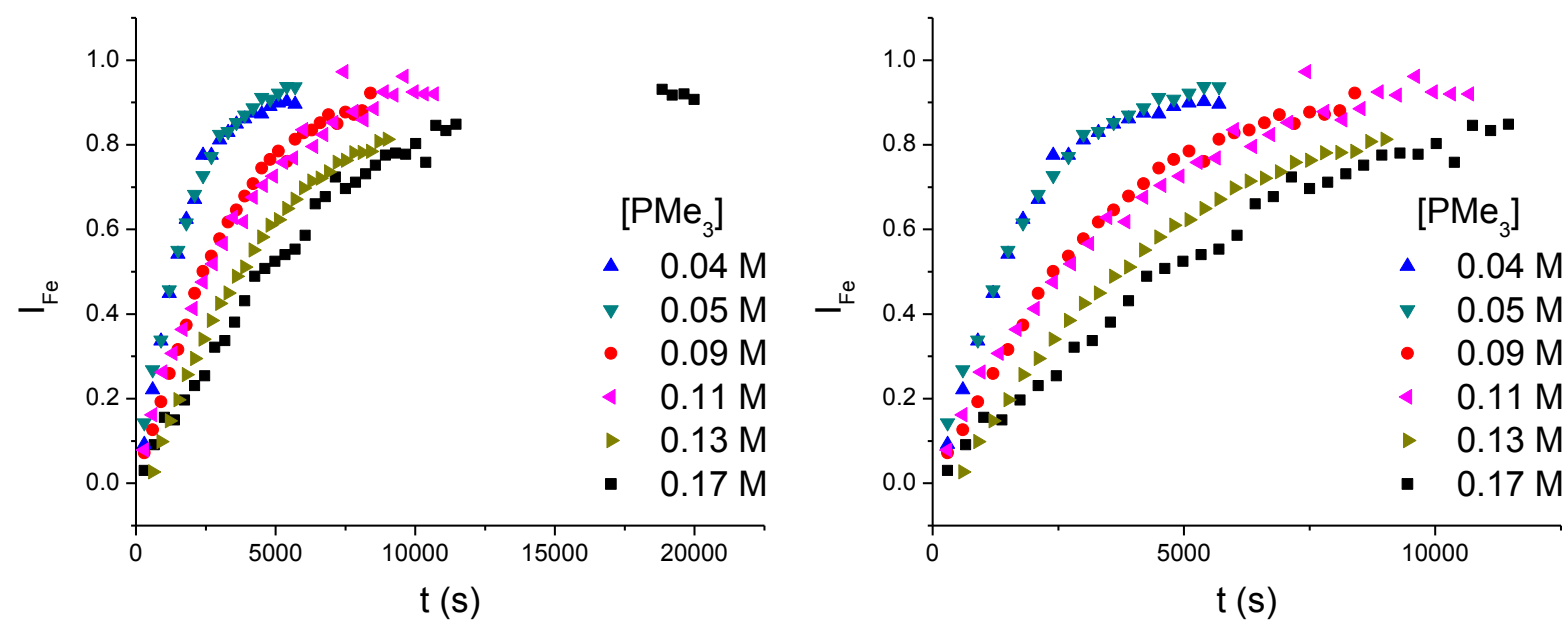

Figure S-6. Representative first-order kinetic plot for the formation of $\mathbf{3 - P M e}$ from the reaction of 1-PMe $\mathrm{PM}_{3}$ with $\mathrm{CO}_{2}(1 \mathrm{~atm})$ in $\mathrm{C}_{6} \mathrm{D}_{6}$ at $23{ }^{\circ} \mathrm{C}$ in the presence of $0.04 \mathrm{M}$ free $\mathrm{PMe}_{3} . k_{\mathrm{obs}}$ $=6.9(3) \times 10^{-4} \mathrm{~s}^{-1}$

A fast pre-equilibrium reaction scheme that is consistent with these results and the known reversible dissociation of $\mathrm{PMe}_{3}$ from 1-PMe 3 is shown in Scheme 6. In Scheme 6, basefree 1 reacts with $\mathrm{CO}_{2}$ to form 3, which, in the presence of $\mathrm{PMe}_{3}$, gives $\mathbf{3}-\mathrm{PMe}_{3}$, while 1-PMe does not react directly with $\mathrm{CO}_{2}$.

The rate law for Scheme 6 is derived below and given by eq 9 . 


$$
\text { Rate }=k_{\mathrm{obs}}[\mathrm{Fe}-\mathrm{Me}]=k_{1}[1] P_{\mathrm{CO} 2}
$$

where $[\mathrm{Fe}-\mathrm{Me}]=\left[\mathbf{1}-\mathbf{P M e}_{3}\right]+[\mathbf{1}]$

$$
\begin{aligned}
& \text { Since } K_{\mathrm{eq}}=\frac{[\mathbf{1}]\left[\mathrm{PMe}_{3}\right]}{\left[1-\mathrm{PMe}_{3}\right]} \\
& {\left[1-\mathrm{PMe}_{3}\right]=\frac{[1]\left[\mathrm{PMe}_{3}\right]}{K_{\mathrm{eq}}}}
\end{aligned}
$$

Therefore,

$$
\begin{aligned}
{[\mathrm{Fe}-\mathrm{Me}]=\left[\mathbf{1}-\mathbf{P M e}_{3}\right]+[\mathbf{1}] } & =\frac{[\mathbf{1}]\left[\mathrm{PMe}_{3}\right]}{K_{\mathrm{eq}}}+[\mathbf{1}] \\
& =\frac{K_{\mathrm{eq}}+\left[\mathrm{PMe}_{3}\right]}{K_{\mathrm{eq}}}[\mathbf{1}]
\end{aligned}
$$

Rearranging,

$$
[\mathbf{1}]=\frac{K_{\mathrm{eq}}}{K_{\mathrm{eq}}+\left[\mathrm{PMe}_{3}\right]}[\mathrm{Fe}-\mathrm{Me}]
$$

And,

$$
\begin{gathered}
\text { Rate }=k_{1}[\mathbf{1}] P_{\mathrm{CO} 2}=\frac{k_{1} K_{\mathrm{eq}}}{K_{\mathrm{eq}}+\left[\mathrm{PMe}_{3}\right]}[\mathrm{Fe}-\mathrm{Me}] P_{\mathrm{CO} 2}=k_{\mathrm{obs}}[\mathrm{Fe}-\mathrm{Me}] \\
\text { where } k_{\mathrm{obs}}=\frac{k_{1} P_{\mathrm{CO} 2} K_{\mathrm{eq}}}{K_{\mathrm{eq}}+\left[\mathrm{PMe}_{3}\right]}
\end{gathered}
$$

Therefore, at $P_{\mathrm{CO} 2}=1 \mathrm{~atm}$,

$$
\frac{1}{k_{\mathrm{obs}}}=\frac{1}{k_{1} K_{\mathrm{eq}}}\left[\mathrm{PMe}_{3}\right]+\frac{1}{k_{1}}
$$

And a plot of $1 / k_{\text {obs }}$ vs $\left[\mathrm{PMe}_{3}\right]$ should be linear with slope $=1 /\left(k_{\mathbf{1}} K_{\text {eq }}\right)$ and y-intercept $=1 / k_{1}$.

This plot is shown in Figure 9 in the text and provides $k_{\mathbf{1}}\left(23{ }^{\circ} \mathrm{C}\right)=2(3) \times 10^{-2} \mathrm{~atm}^{-1} \mathrm{~s}^{-1}$ and $K_{\text {eq }}\left(23{ }^{\circ} \mathrm{C}\right)=2(3) \times 10^{-3} \mathrm{M}$. These values are in good agreement with the $k_{1}$ and $K_{\text {eq }}$ values 
determined independently by the studies of 1 discussed above: $k_{1}=2(4) \times 10^{-2} \mathrm{~atm}^{-1} \mathrm{~s}^{-1}$ and $K_{\text {eq }}$ $=1.8(9) \times 10^{-3} \mathrm{M}$

Kinetic Analysis of the Reaction of 2 with $\mathbf{C O}_{2}$. A valved J. Young NMR tube was charged with $2(8.7 \mathrm{mg}, 0.010 \mathrm{mmol}) . \mathrm{C}_{6} \mathrm{D}_{5} \mathrm{~F}(0.5 \mathrm{~mL})$ was added by vacuum transfer at -196 ${ }^{\circ} \mathrm{C}$. The mixture was thawed at $0{ }^{\circ} \mathrm{C}$ and exposed to $\mathrm{CO}_{2}(1 \mathrm{~atm}, 11$ equiv). The tube was then rapidly inserted into an NMR probe that had been pre-cooled at $0{ }^{\circ} \mathrm{C} .{ }^{1} \mathrm{H}$ NMR spectra were recorded periodically. Representative spectra are shown in Figure 11 in the text.

The rate equation for the conversion of $\mathbf{2}$ to $\mathbf{4}$ is given by eq 11 .

$$
\text { Rate }=k_{2}{ }^{\prime}[2]=k_{2}[2] P_{\mathrm{CO} 2}
$$

where $k_{2}{ }^{\prime}=$ observed first-order rate constant

and $k_{2}=$ second-order rate constant.

The logarithmic form of the first-order rate equation for the disappearance of $\mathbf{2}$ is given by eq 12 .

$\ln \left(I_{2}\right)=-k_{2}{ }^{\prime} t+\ln \left(I_{2,0}\right)$

where $I_{2}=$ integral value of the $\delta-25.0$ resonance of 2 relative to the pentane resonance $(\delta 1.2$, internal standard) at time $=\mathrm{t} ; I_{2,0}=$ integral value of the $\delta-25.0$ resonance of 2 relative to the pentane resonance at the start of the reaction

First-order kinetic plots were generated using the program Origin 8. A representative plot for the disappearance of 2 at $0{ }^{\circ} \mathrm{C}$ and $P_{\mathrm{CO} 2}=1$ atm is shown in Figure $\mathrm{S}-7 \mathrm{a} . k_{\mathbf{2}}{ }^{\prime}\left(0^{\circ} \mathrm{C}\right)=$ $7.10(9) \times 10^{-4} \mathrm{~s}^{-1}$.

The first-order rate equation for the appearance of $\mathbf{4}$ (logarithmic form) is given by eq 13 . $\ln \left(I_{4, \infty}-I_{4}\right)=-k_{2}{ }^{\prime} \mathrm{t}+\ln \left(I_{4, \infty}\right)$ 
where $I_{4, \infty}=$ integral value of the $\delta$-30.5 resonance of 4 relative to the pentane resonance $(\delta 1.2$, internal standard) at the end of reaction. $I_{4}=$ integral value of the $\delta-30.5$ resonance of 4 relative to the pentane resonance at time $=\mathrm{t}$

The first-order plot for the appearance of $\mathbf{4}$ is shown Figure S-7b. The value for $k_{2}$ ' determined from this plot, $k_{2}{ }^{\prime}\left(0^{\circ} \mathrm{C}\right)=8.3(3) \times 10^{-4} \mathrm{~s}^{-1}$, is in good agreement with the value determined from the disappearance of 2.

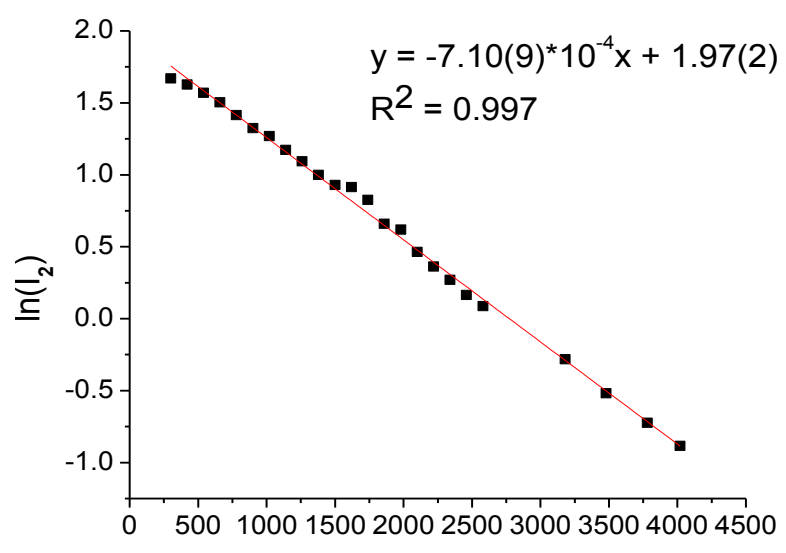

$\mathrm{t}$ (s)

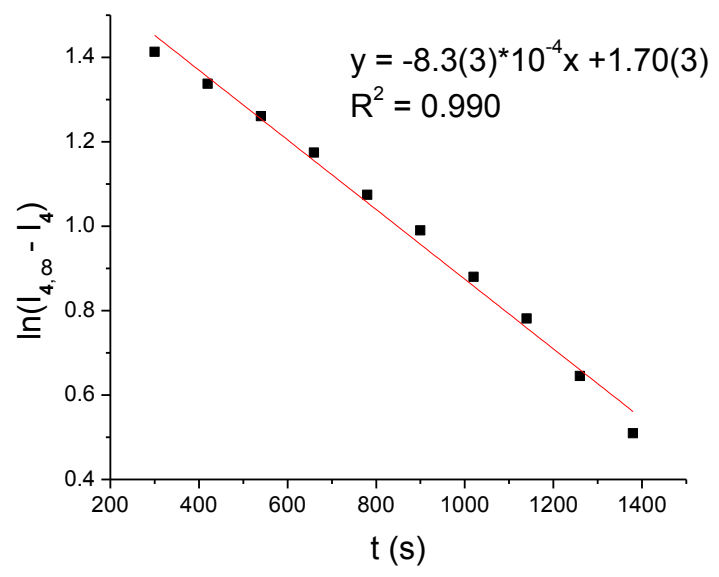

Figure S-7. Representative first-order kinetic plots for the reaction of 2 with $\mathrm{CO}_{2}$ in $\mathrm{C}_{6} \mathrm{D}_{5} \mathrm{~F}(0$ $\left.{ }^{\circ} \mathrm{C}, P_{\mathrm{CO} 2}=1 \mathrm{~atm}\right)$. A plot for the disappearance of 2 is shown on the left $\left(k_{2}{ }^{\prime}=7.10(9) \times 10^{-4} \mathrm{~s}^{-1}\right)$. A plot for the appearance of 4 is shown on the right $\left(k_{2}{ }^{\prime}=8.3(3) \times 10^{-4} \mathrm{~s}^{-1}\right)$.

The first-order rate constants for the conversion of $\mathbf{2}$ to $\mathbf{4}$ were obtained at $0{ }^{\circ} \mathrm{C}$ over the pressure range of $0-6.1$ atm. A plot of $k_{2}{ }^{\prime}$ vs. $P_{\mathrm{CO} 2}$ is shown on the right of Figure S-8. This plot establishes that the reaction is first order in $P_{\mathrm{CO} 2}$ and provides $k_{2}=8.8(5) \times 10^{-4} \mathrm{~atm}^{-1} \mathrm{~s}^{-1}$. 


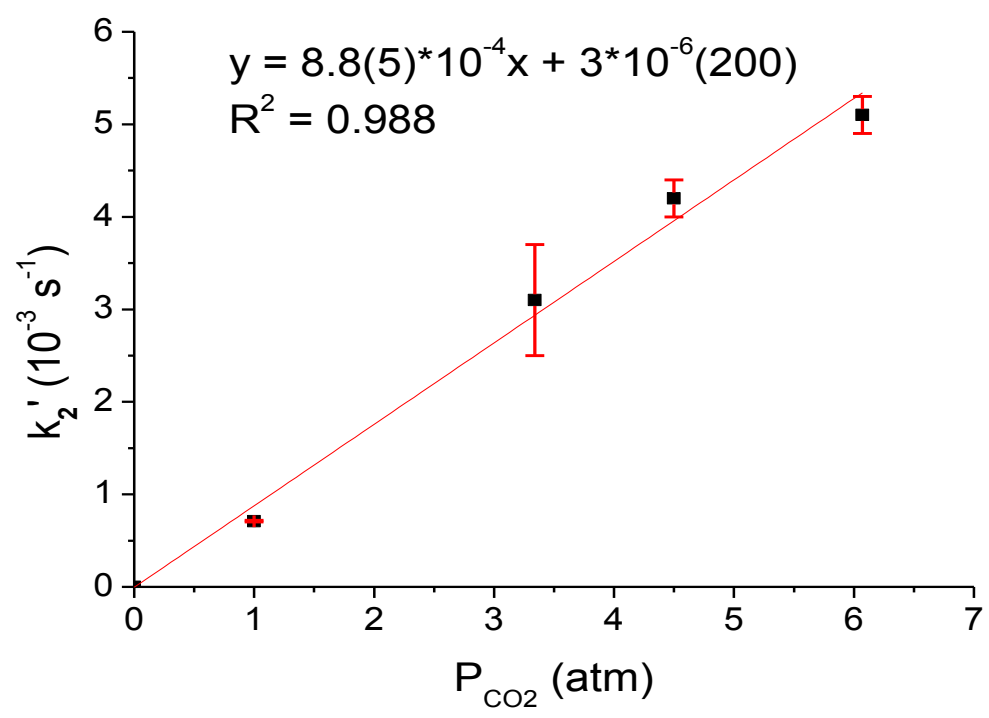

Figure S-8. A plot of $k_{2}$ ' vs $P_{\mathrm{CO} 2}$ for the reaction of 2 with $\mathrm{CO}_{2}$ in $\mathrm{C}_{6} \mathrm{D}_{5} \mathrm{~F}$ at $0{ }^{\circ} \mathrm{C}$.

The second-order rate constants for the conversion of $\mathbf{2}$ to $\mathbf{4}$ were obtained at $P_{\mathrm{CO} 2}=1$ atm over the temperature range of $-10^{\circ} \mathrm{C}-23^{\circ} \mathrm{C}$. The Eyring plot for the conversion of 2 to 4 is shown in Figure S-9 and activation parameters are listed in Table 1 in the text.

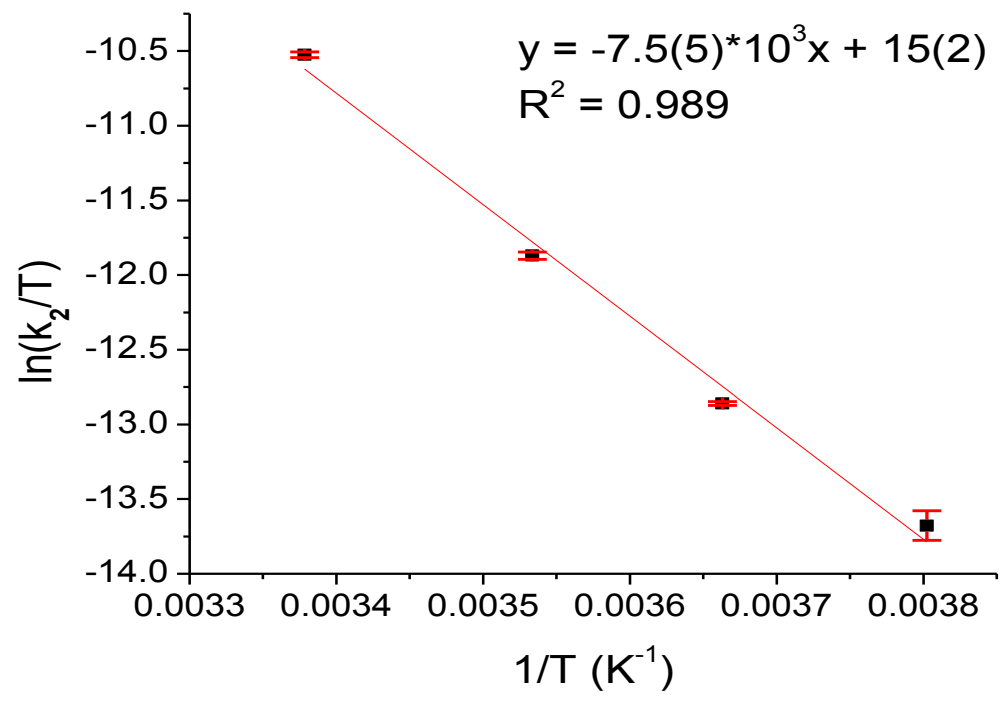

Figure S-9. The Eyring plot for the reaction of 2 with $\mathrm{CO}_{2}\left(1\right.$ atm) to produce 4 in $\mathrm{C}_{6} \mathrm{D}_{5} \mathrm{~F}$. 


\section{IR Assignments}

The $v_{\mathrm{CO}}$ bands for $\mathbf{3}$ were assigned by inspection of the IR spectra of $\mathbf{3}, \mathbf{3 -}^{\mathbf{1 3}} \mathbf{C}_{\mathbf{1}}$, which was synthesized from $\mathbf{1}+{ }^{13} \mathrm{CO}_{2}$, and (PDI)FeCl (Figure S-10), and those for $\mathbf{4}$ were assigned by inspection of the IR spectra of $\mathbf{4}, \mathbf{4 -}^{\mathbf{1 3}} \mathbf{C}_{\mathbf{1}}$, which was synthesized from $\mathbf{2}+{ }^{13} \mathrm{CO}_{2}$, and (PDI)FeCl $\mathrm{Fe}_{2}$ (Figure S-11).

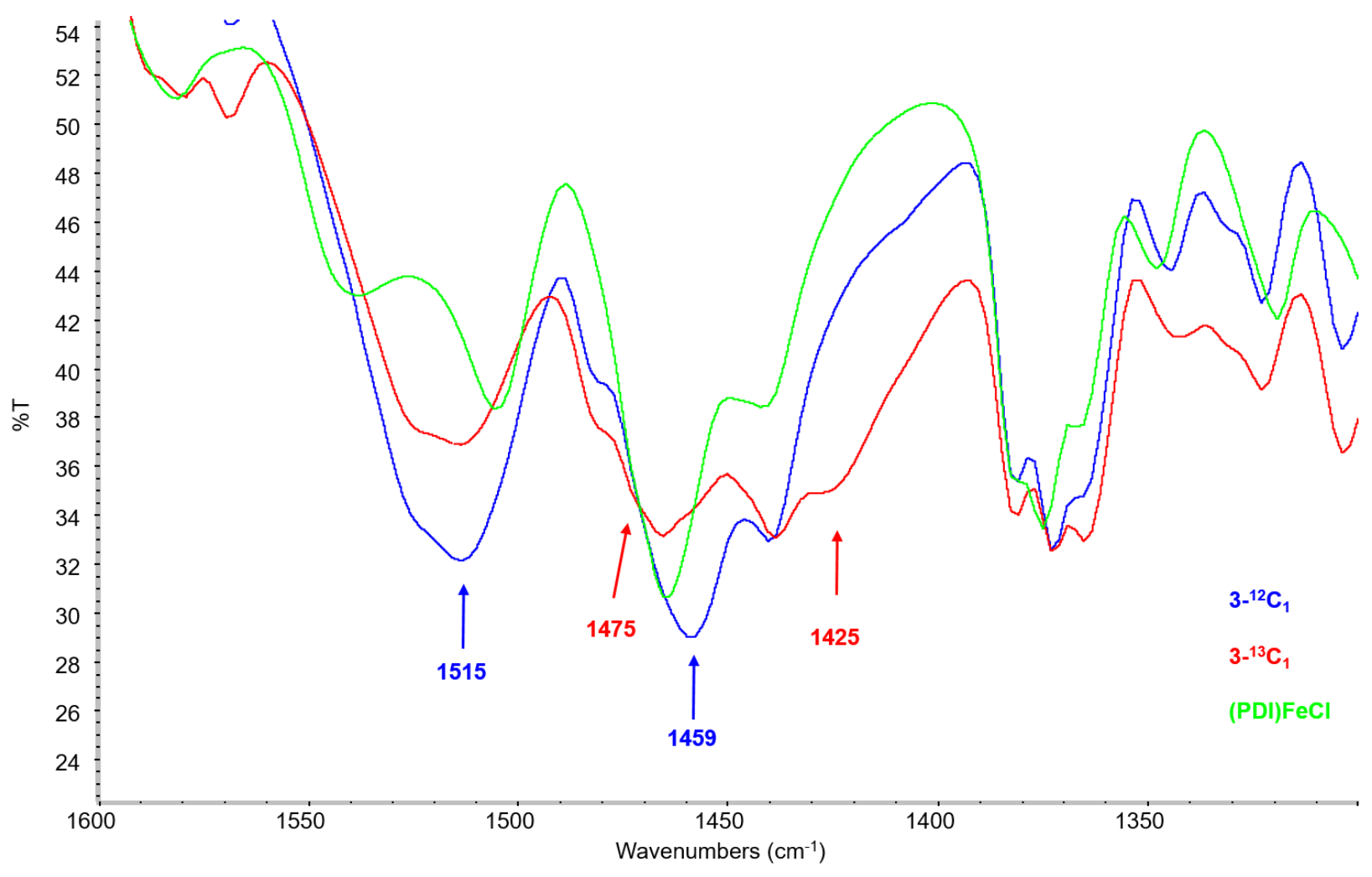

Figure S-10. IR spectra of $\mathbf{3}, \mathbf{3 -}^{\mathbf{1 3}} \mathbf{C}_{\mathbf{1}}$ and (PDI)FeCl (KBr pellet). 


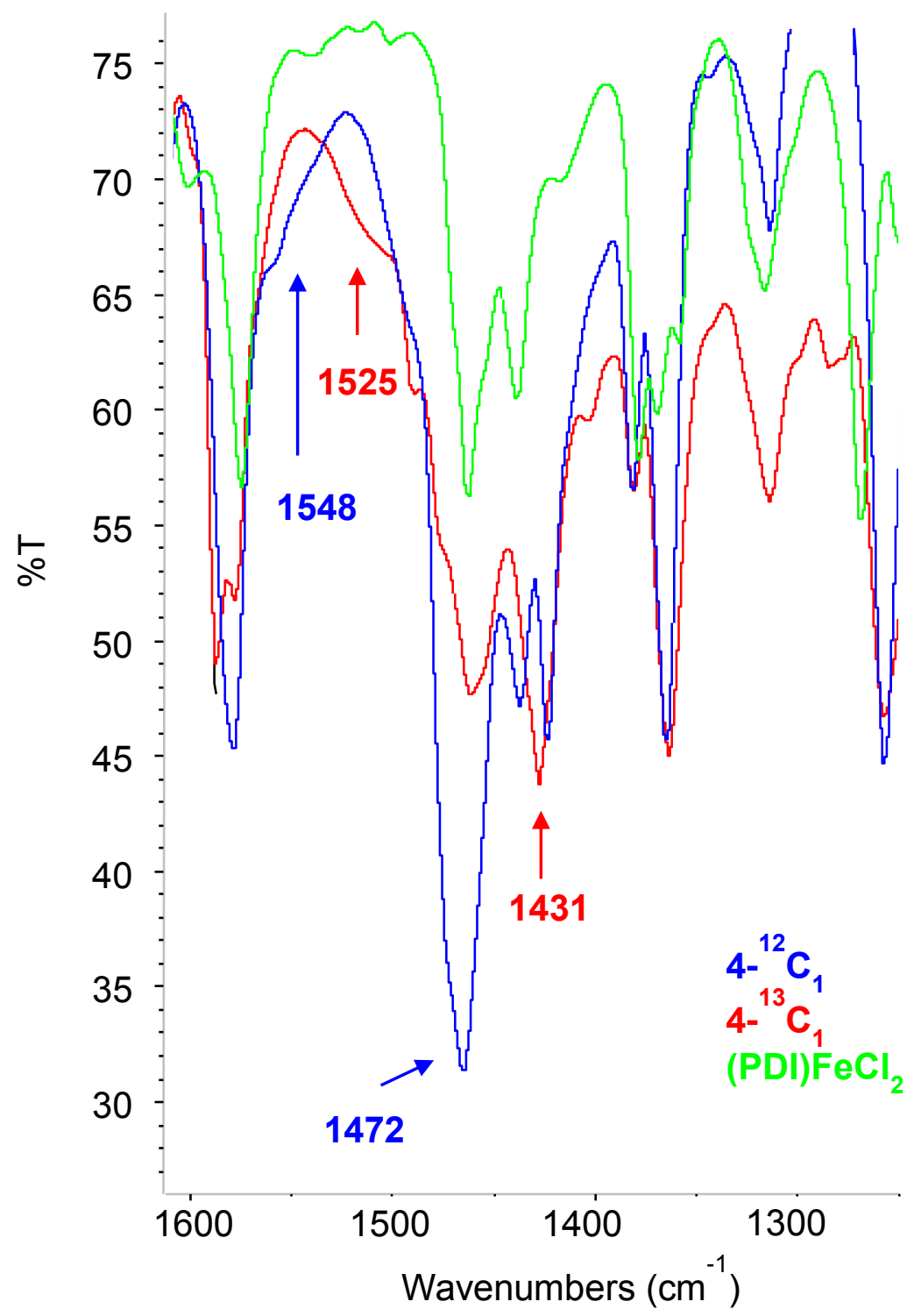

Figure S-11. IR spectra of 4, 4- ${ }^{\mathbf{1 3}} \mathrm{C}_{\mathbf{1}}$ and (PDI)FeCl 2 ( $\mathrm{KBr}$ pellet). 


\section{Temperature-Dependent SQUID Magnetization Data}

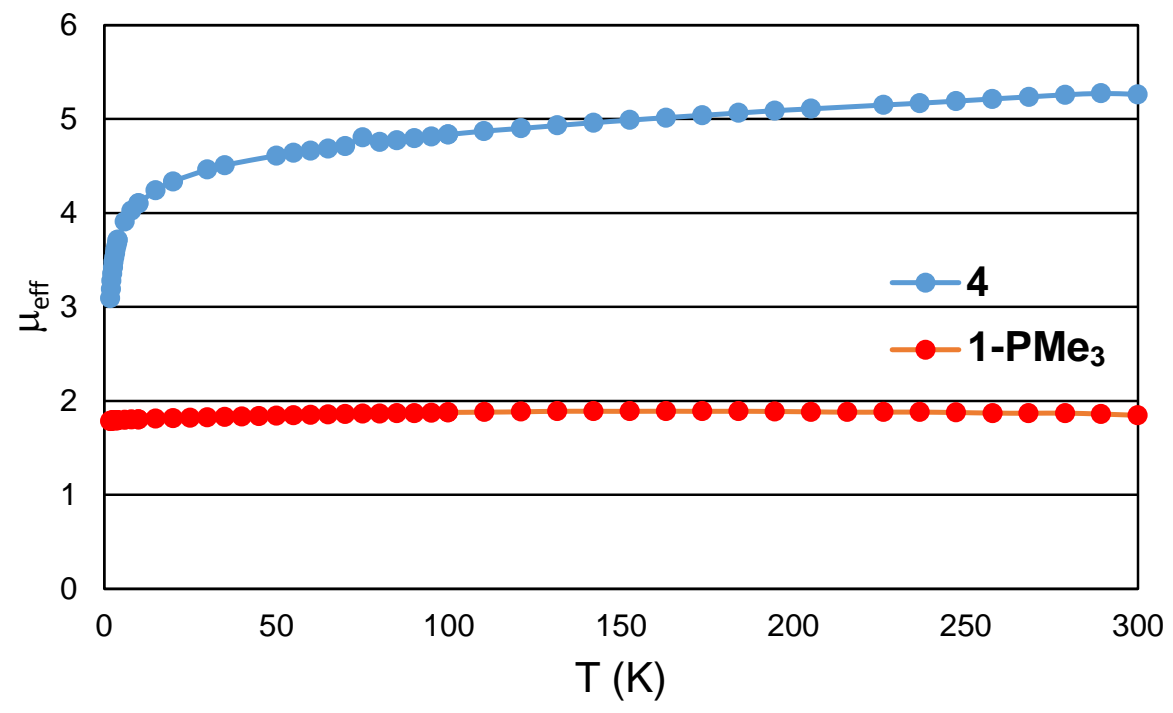

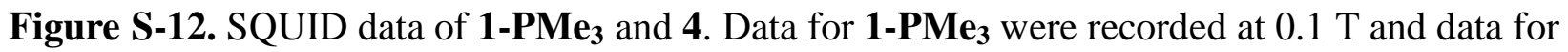
4 were recorded at $1 \mathrm{~T}$. 


\section{EPR Spectrum of 1-PMe}

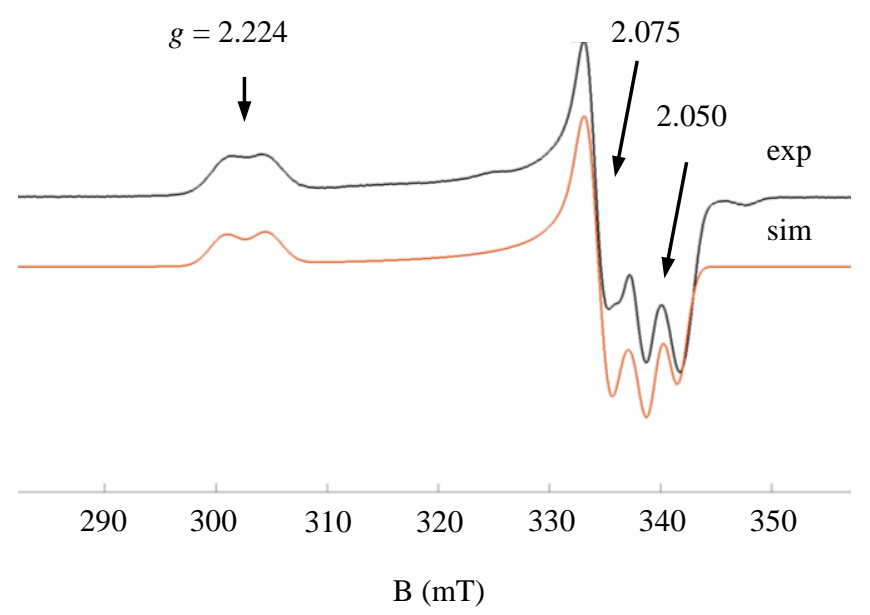

Figure S-13. The X-band EPR spectrum of $\mathbf{1 - P M e} \mathbf{e}_{3}$ in a toluene glass at $15 \mathrm{~K}$. The experimental spectrum is shown in black and the simulated spectrum is shown in red. 
VII. NMR Spectra for 1-PMe 3 , 2-THF, 3-PMe 3 , 4, and 4-THF

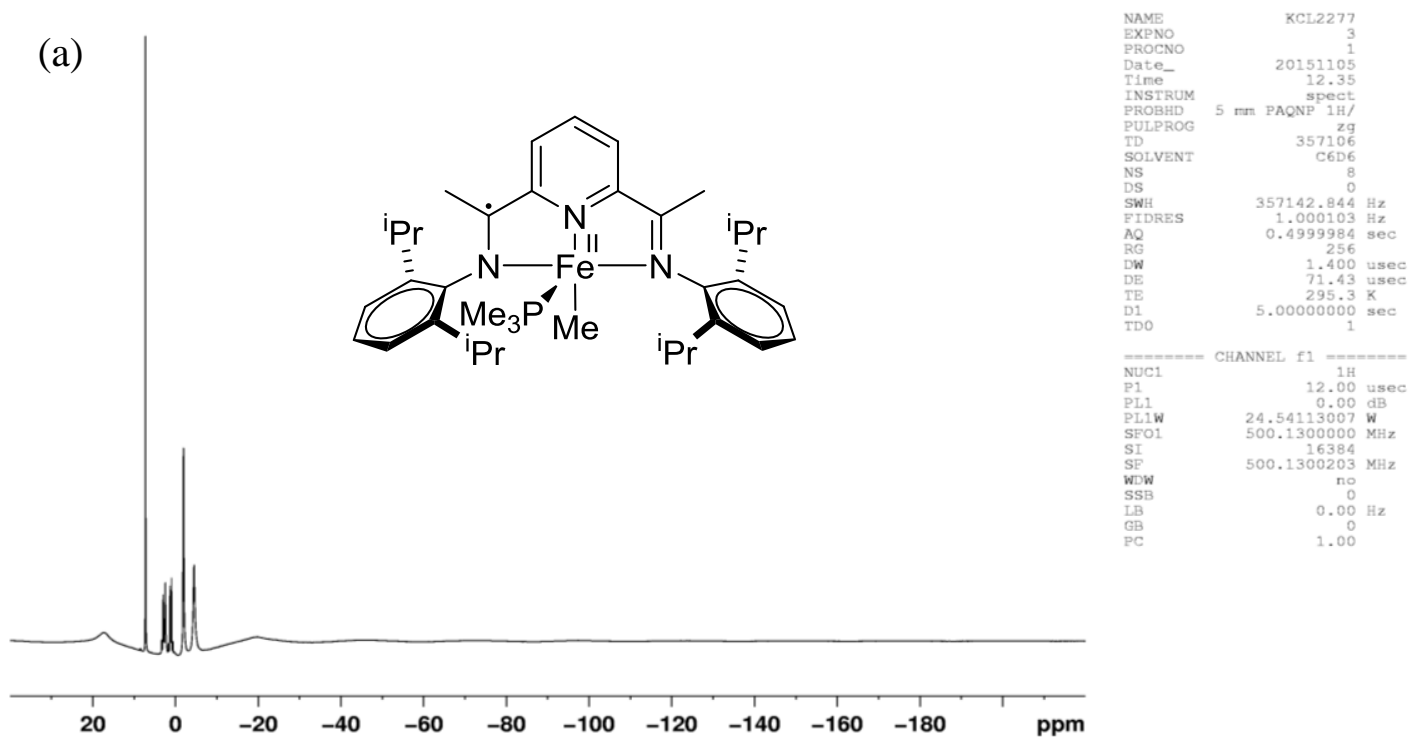

(b)

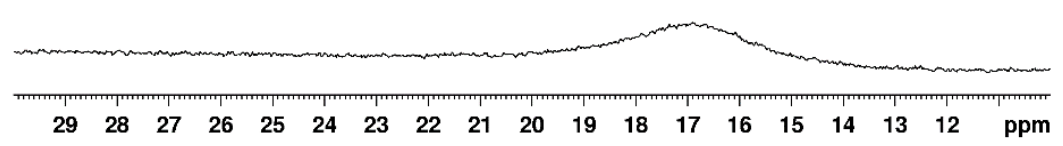

(c)

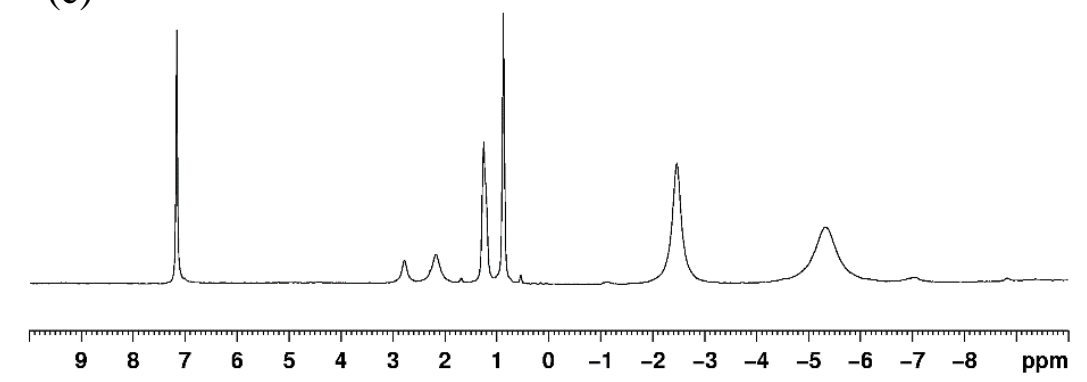

(d)

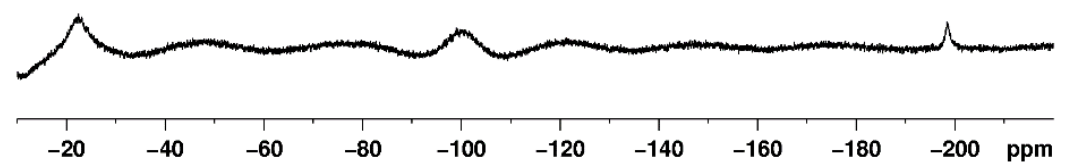

Figure S-14. ${ }^{1} \mathrm{H}$ NMR of 1-PMe ${ }_{3}$ in $\mathrm{C}_{6} \mathrm{D}_{6}(0.027 \mathrm{M})$. (a) The full spectrum, and expansions of key regions: (b) $\delta 30-10$, (c) $\delta 10--10$, and (d) $\delta-10--220$ are shown. 
(a)

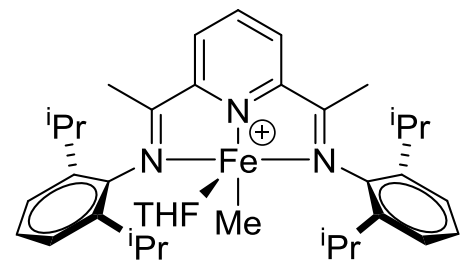

$\ominus$
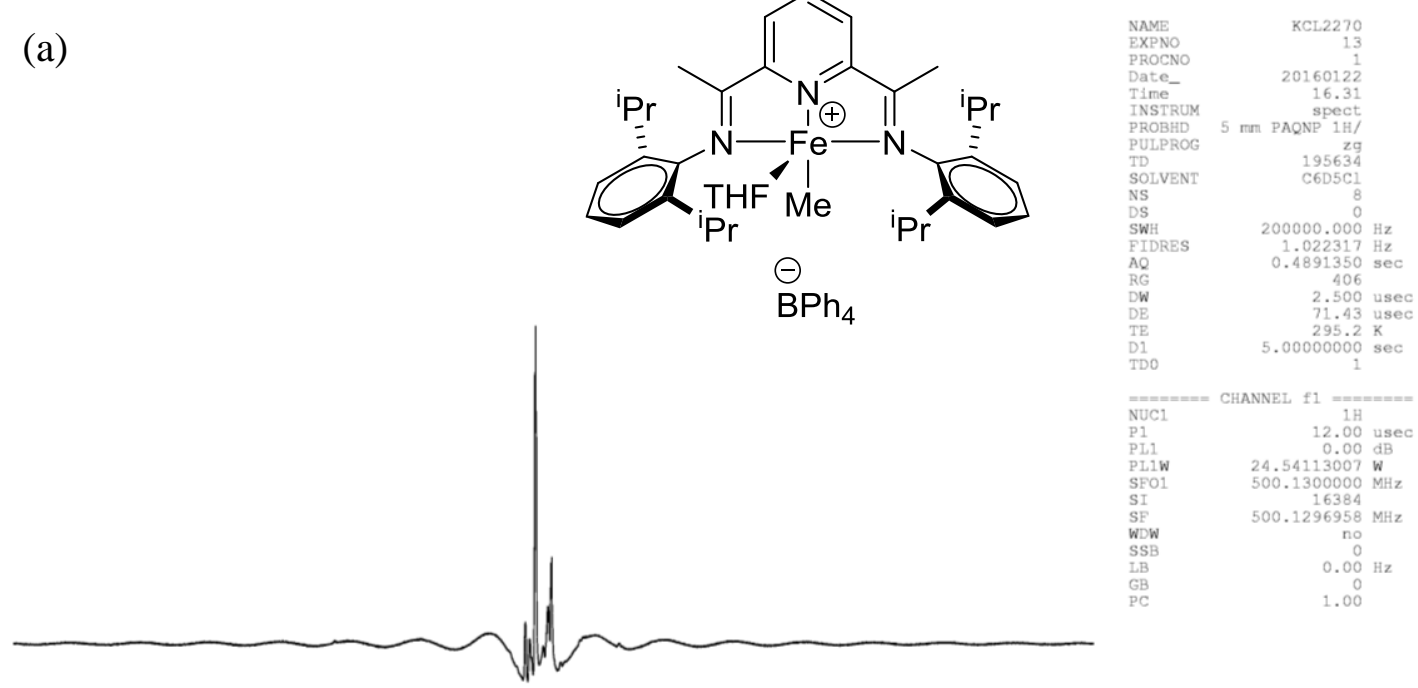

(b)
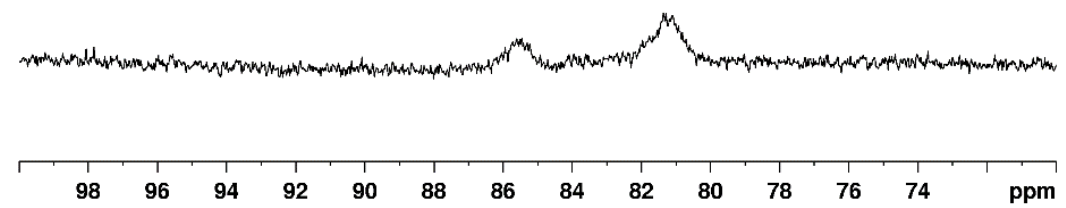

(c)

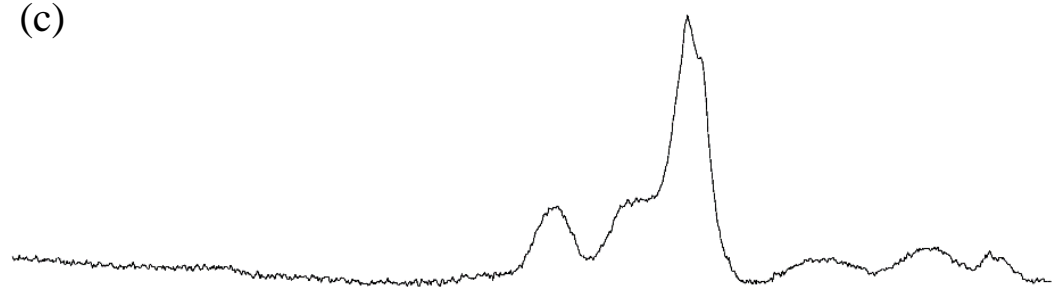

$\begin{array}{lllllllllllllllllll}19 & 18 & 17 & 16 & 15 & 14 & 13 & 12 & 11 & 10 & 9 & 8 & 7 & 6 & 5 & 4 & 3 & 2 & \mathrm{ppm}\end{array}$ 


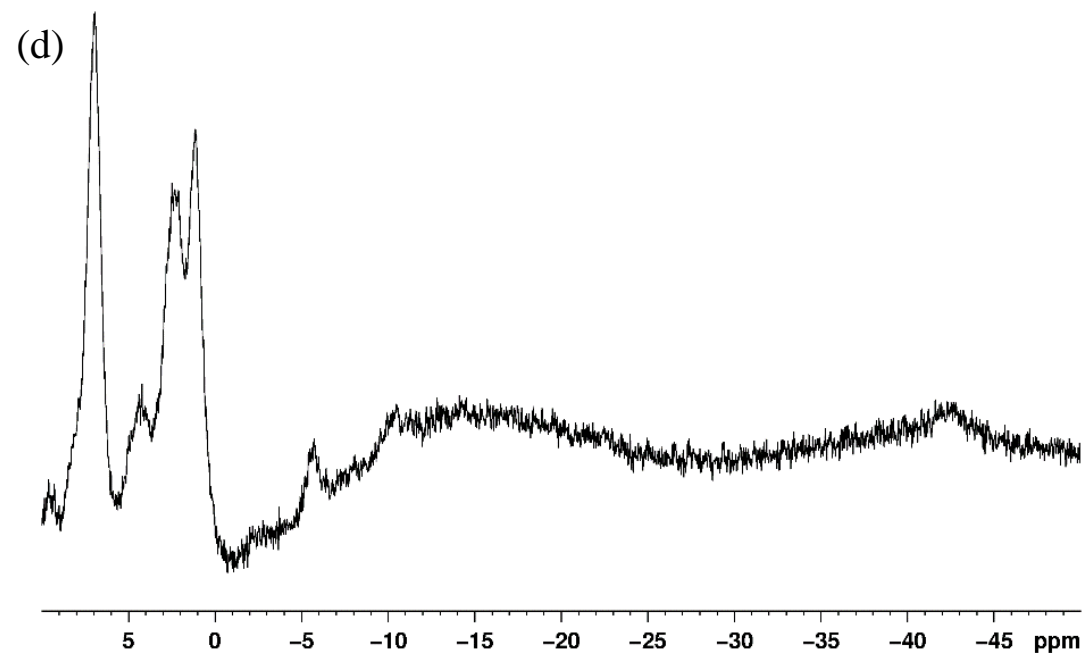

Figure S-15. ${ }^{1} \mathrm{H}$ NMR of 2-THF in $\mathrm{C}_{6} \mathrm{D}_{5} \mathrm{~F}$. (a) The full spectrum, and expansions of key regions: (b) $\delta 100-70$, (c) $\delta 20-0$, and (d) $\delta 10--50$ are shown. 

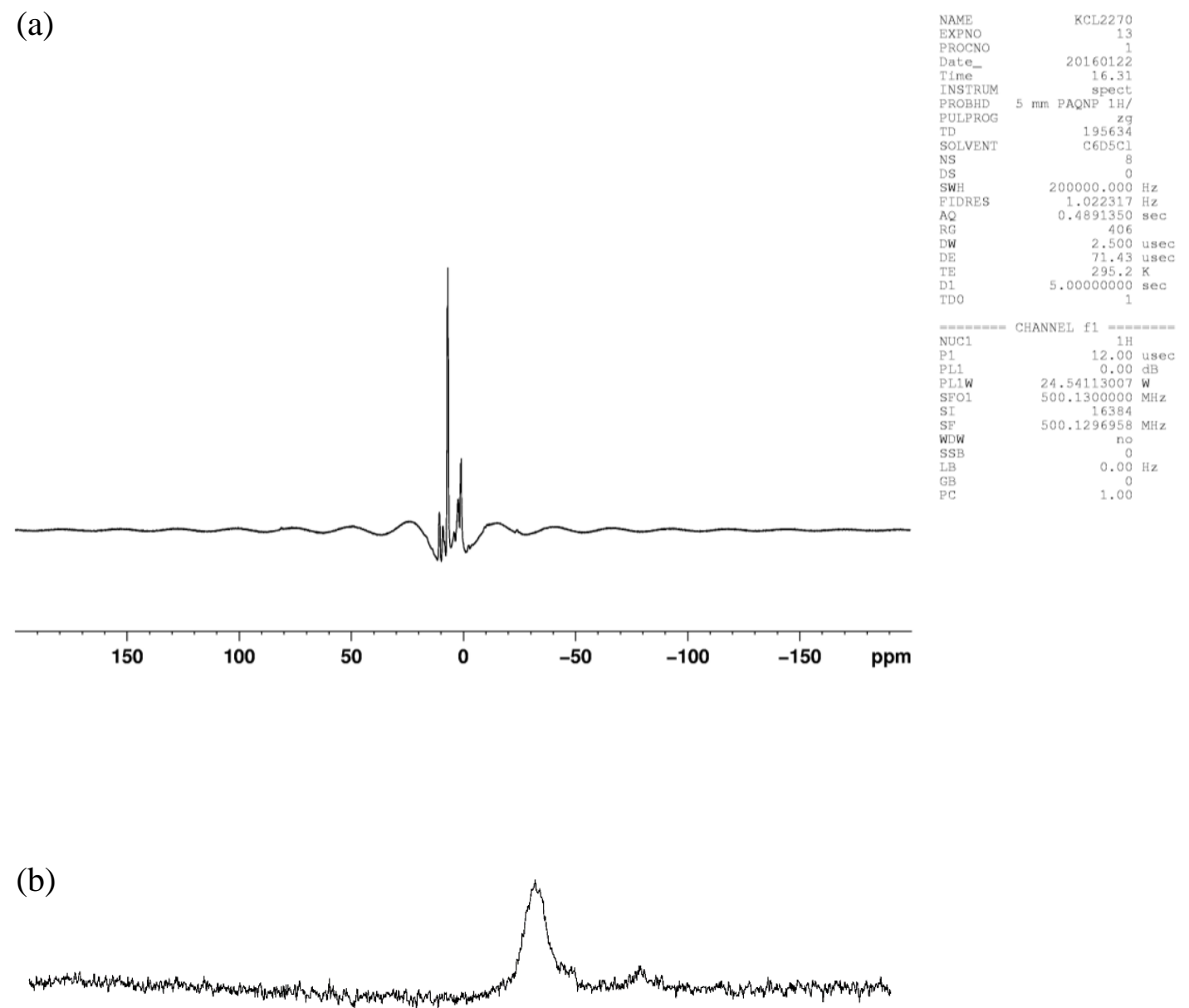

\begin{tabular}{|llllllllllllll}
\hline & & & & & & & & & & & & & \\
98 & 96 & 94 & 92 & 90 & 88 & 86 & 84 & 82 & 80 & 78 & 76 & 74 & $\mathrm{ppm}$
\end{tabular}

(c)

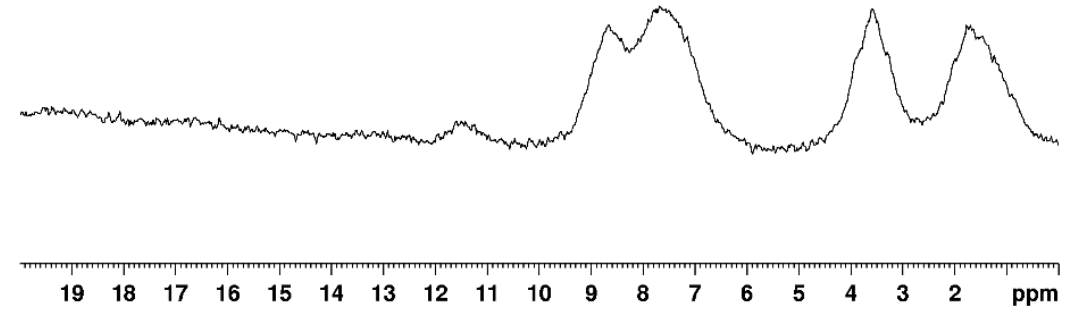


(d)

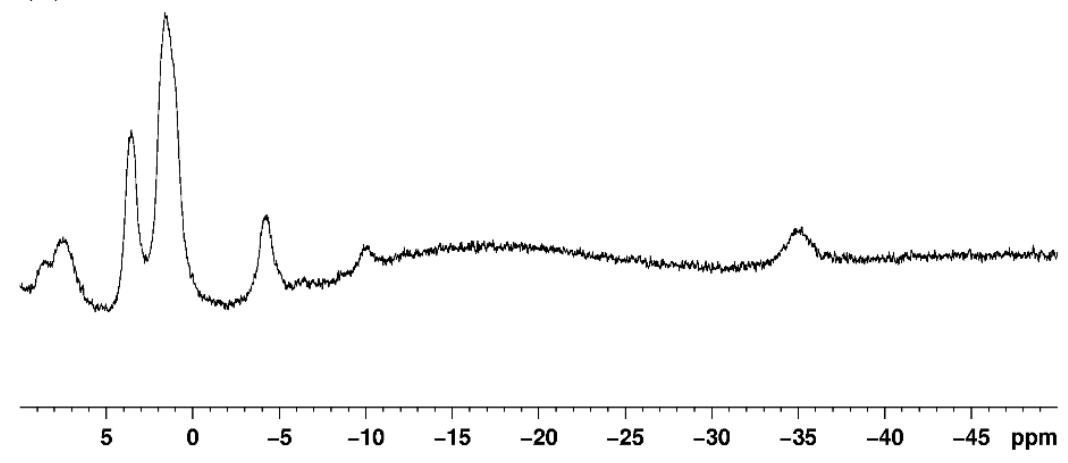

Figure S-16. ${ }^{1} \mathrm{H}$ NMR of 2-THF in THF- $d_{8}$. (a) The full spectrum, and expansions of key regions: (b) $\delta 100-70$, (c) $\delta 20-0$, and (d) $\delta 10--50$ are shown. 

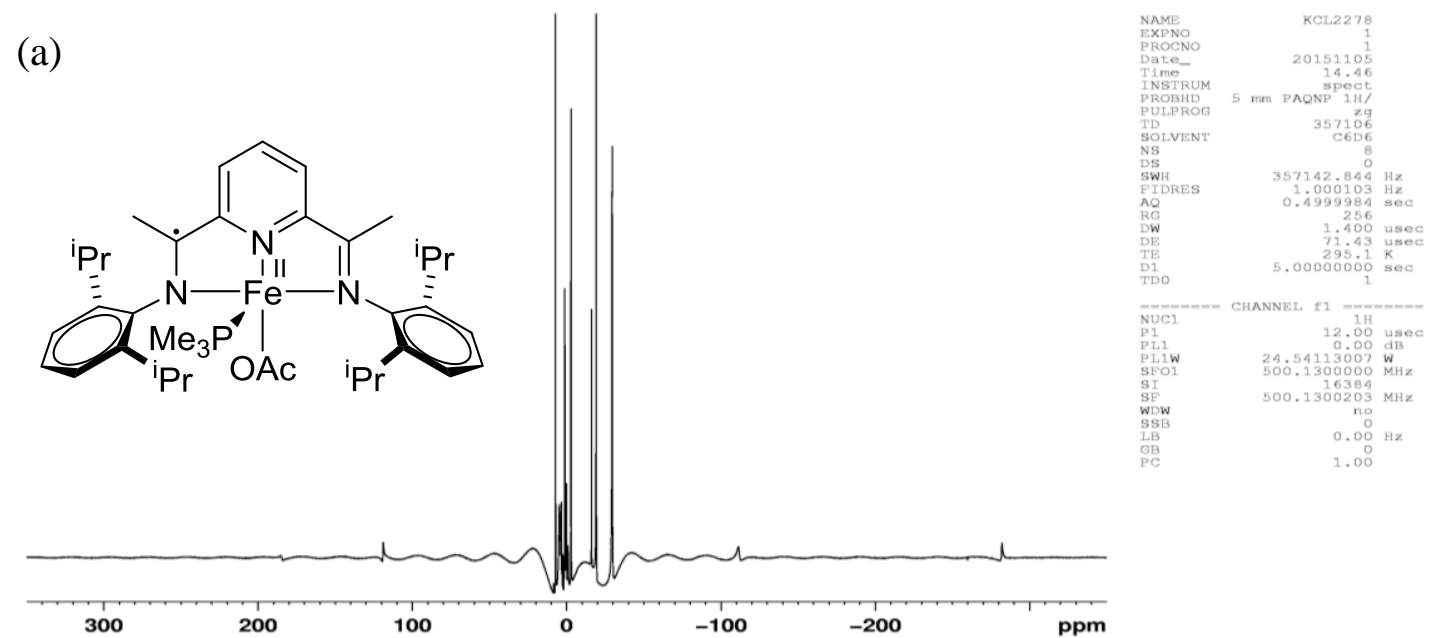

(b)

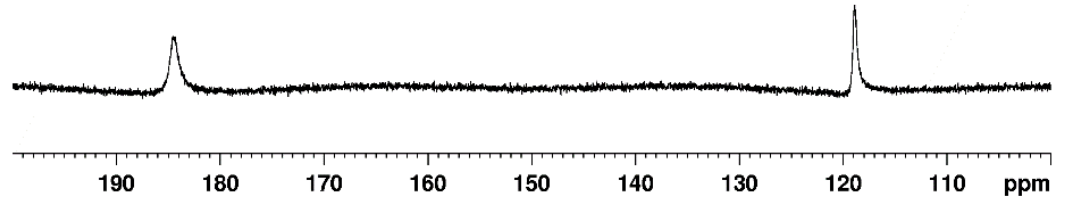

(c)
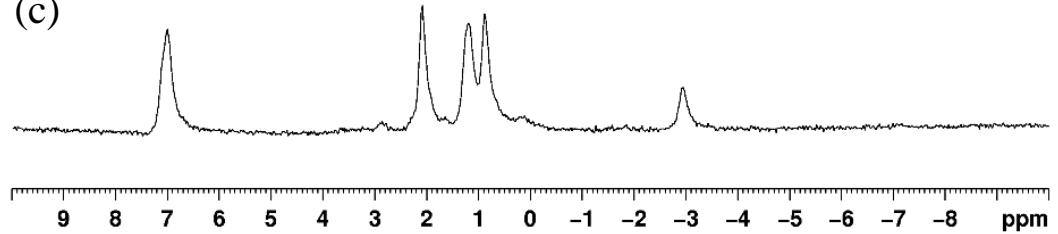

(d)
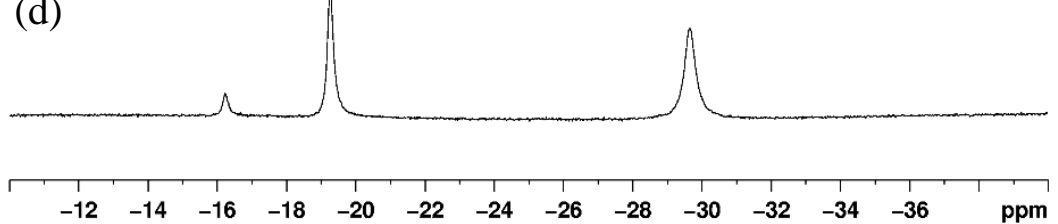

(e)

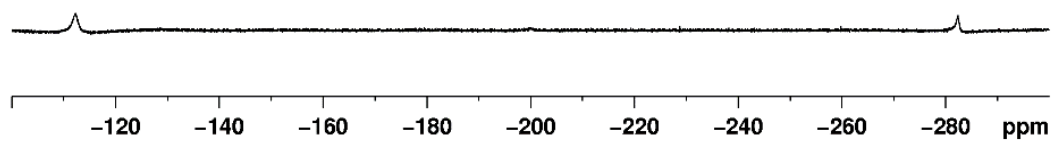

Figure S-17. ${ }^{1} \mathrm{H}$ NMR of 3-PMe 3 in toluene- $d_{8}(0.017 \mathrm{M})$. (a) The full spectrum, and expansions of key regions: (b) $\delta 200-100$, (c) $\delta 10--10$, (d) $\delta-10--40$, and (e) $\delta-100--300$ are shown. 
(a)
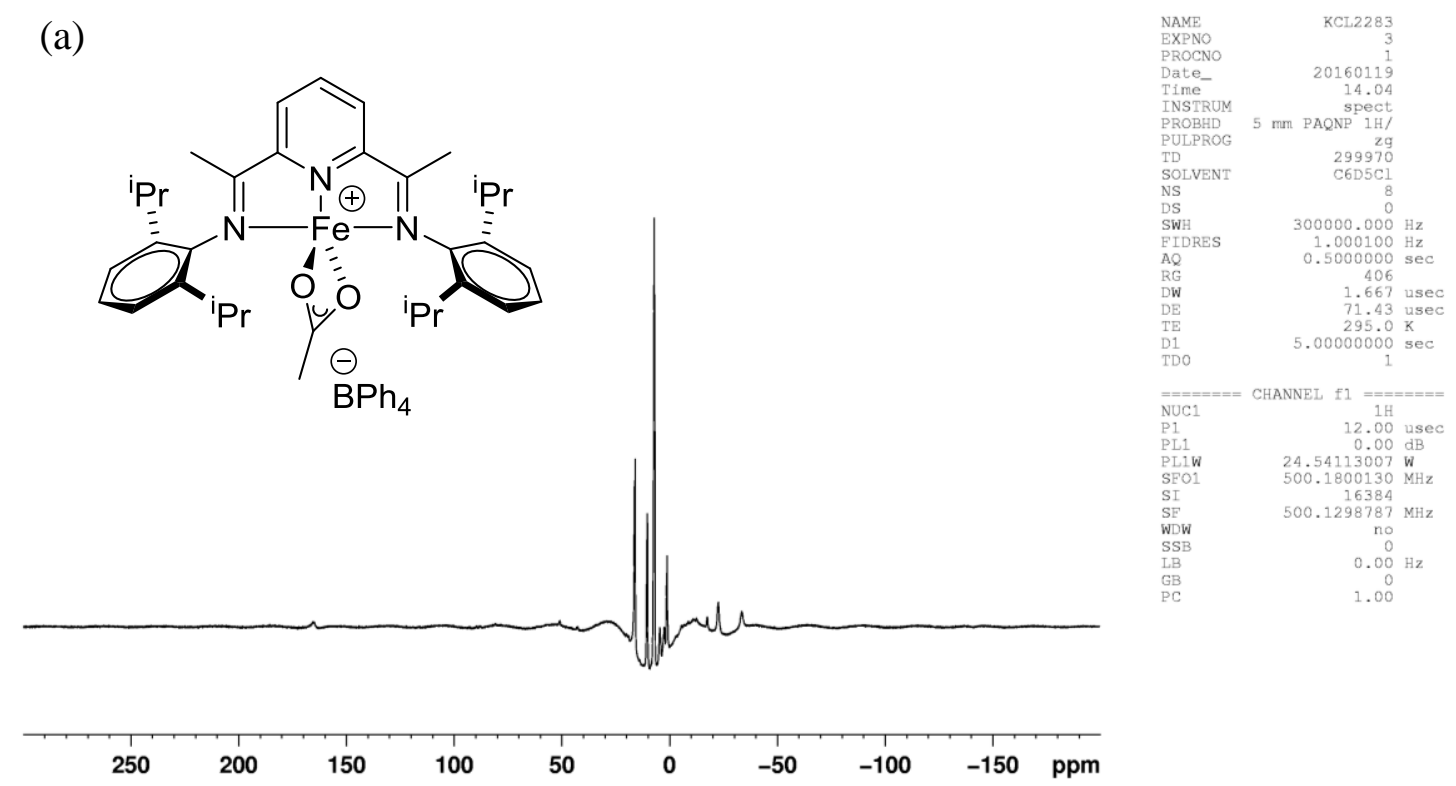

(b)

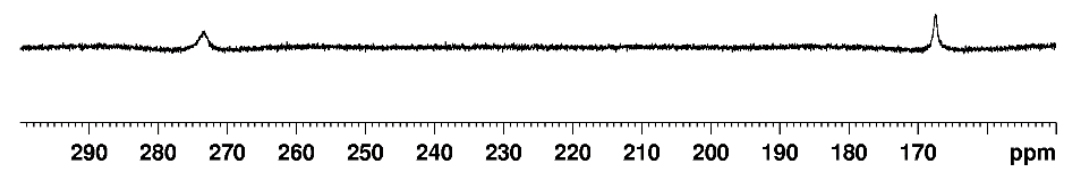

(c)
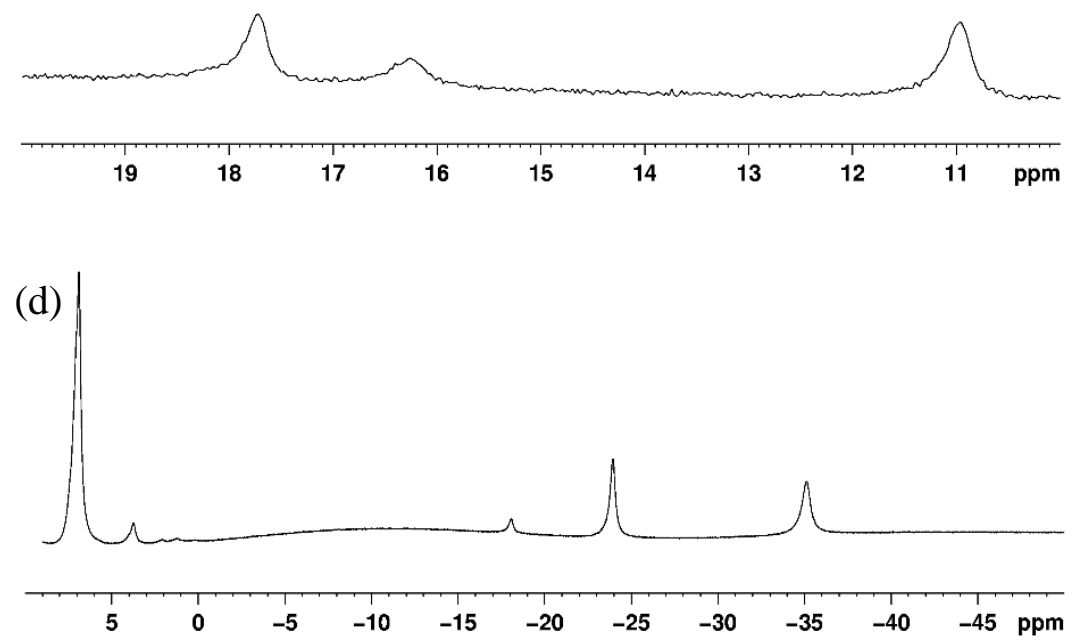
(e)

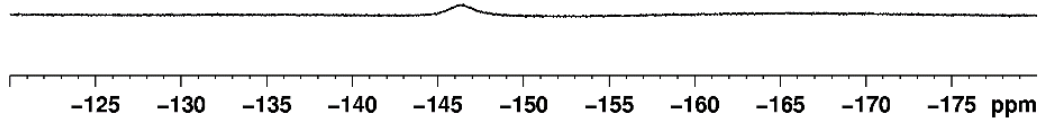

Figure S-18. ${ }^{1} \mathrm{H}$ NMR of $4\left(\mathrm{C}_{6} \mathrm{D}_{5} \mathrm{~F}\right)$. (a) The full spectrum, and expansions of key regions: (b) $\delta$ $300-150$, (c) $\delta 20-10$, (d) $\delta 10--50$, and (e) $\delta-120--180$ are shown. 


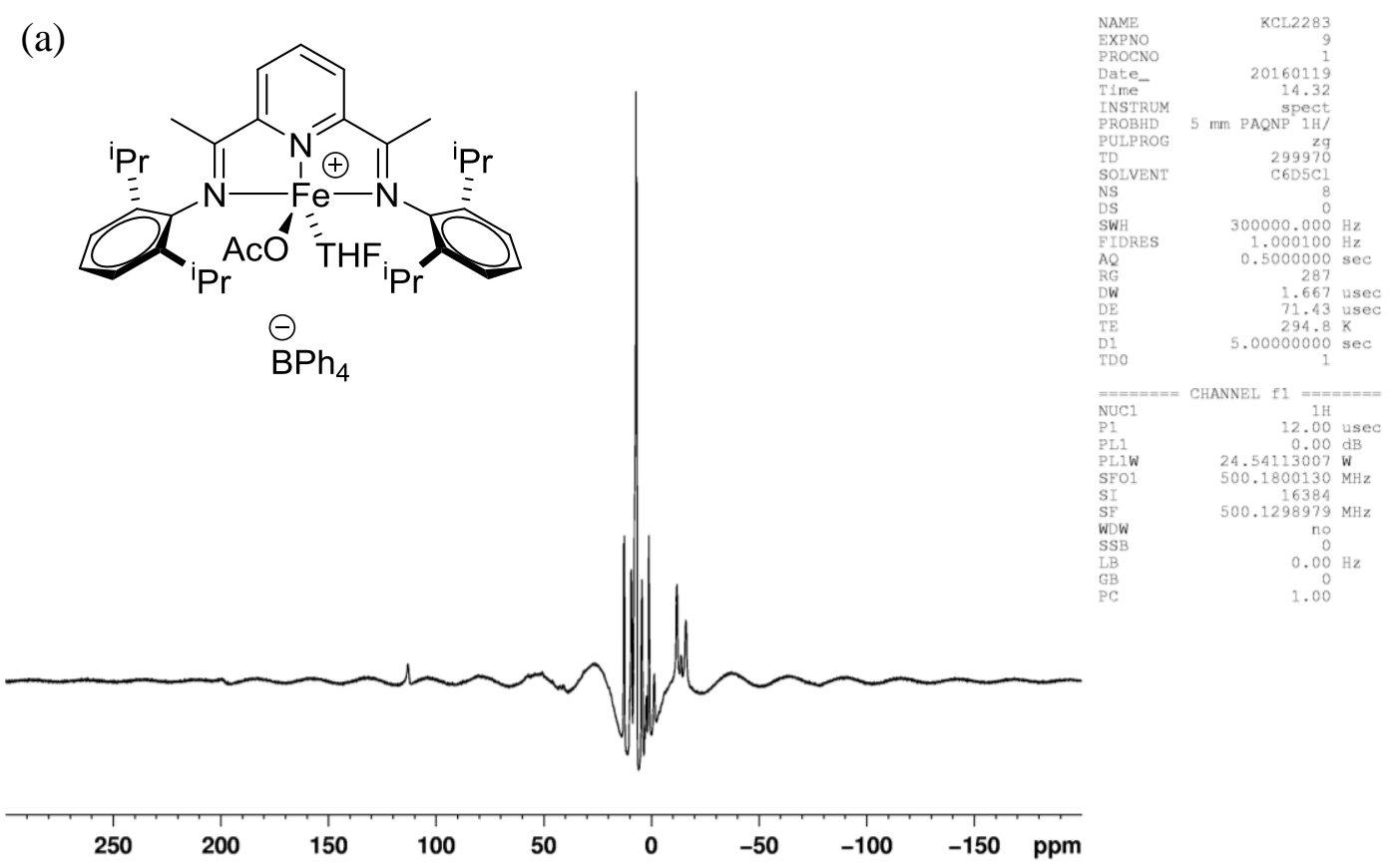

(b)

(c)

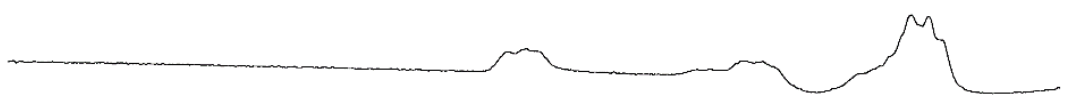



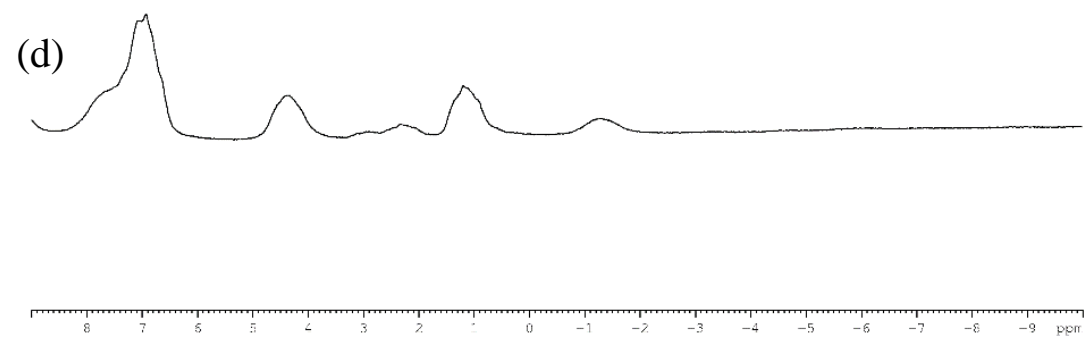

(e)
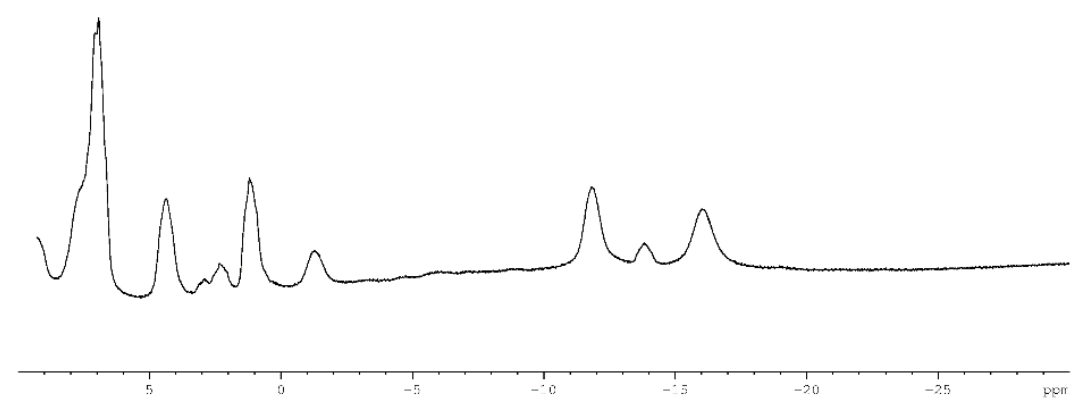

(f)

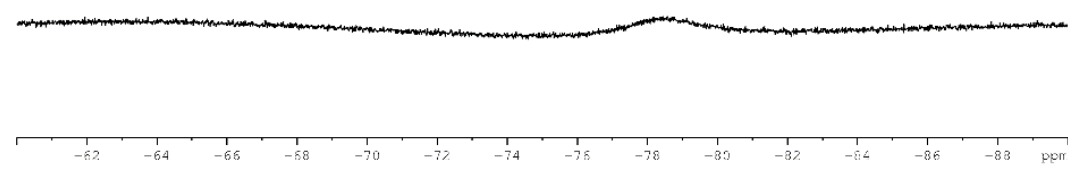

Figure S-19. ${ }^{1} \mathrm{H}$ NMR of 4-THF $\left(\mathrm{C}_{6} \mathrm{D}_{5} \mathrm{~F}\right)$. (a) The full spectrum, and expansions of key regions:

(b) $\delta 220-100$, (c) $\delta 20-5$, (d) $\delta 9--10$, (e) $\delta 10--30$, and (f) $\delta-60--90$ are shown. 


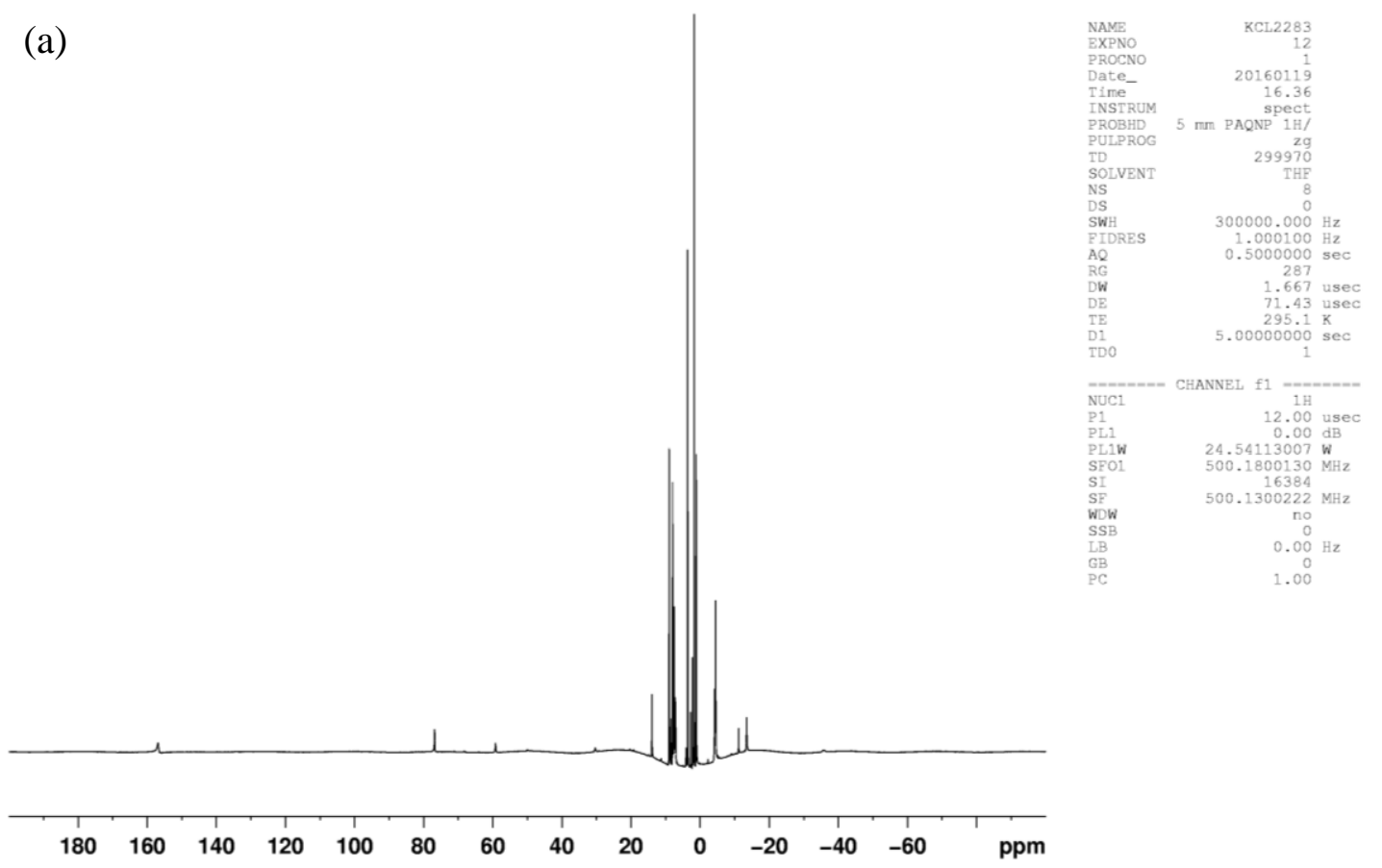

(b)

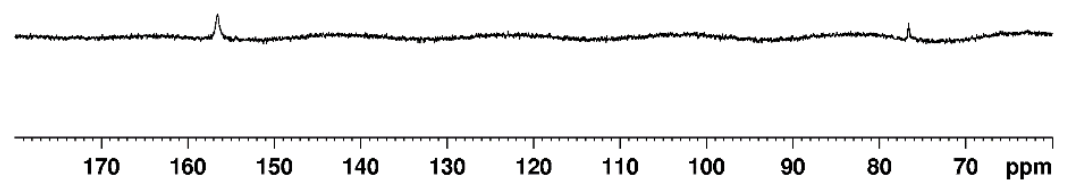

(c)

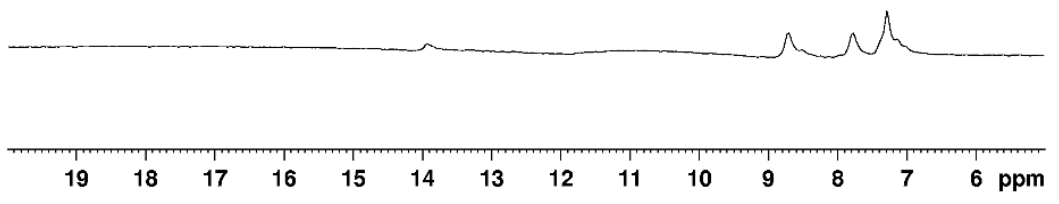




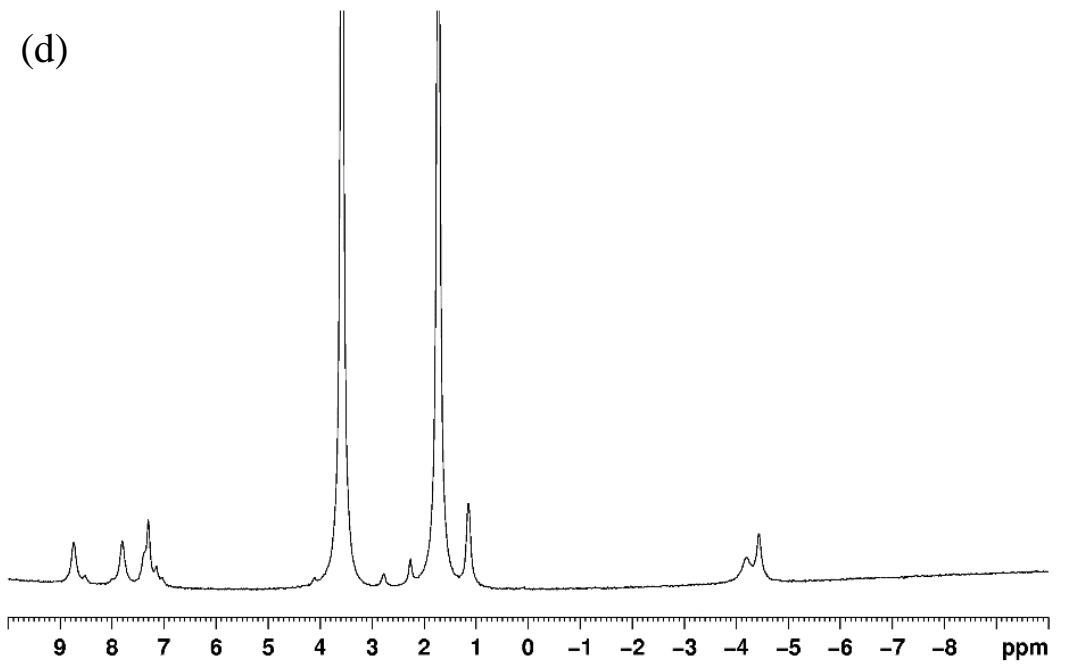

(e)

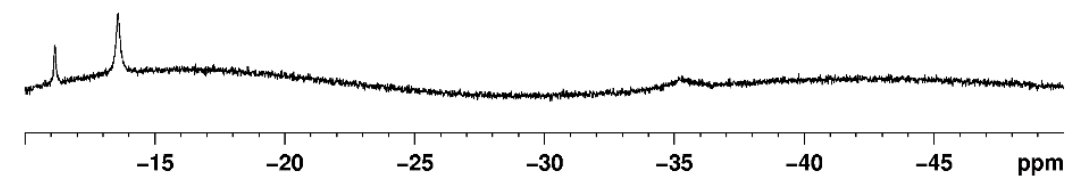

Figure S-20. ${ }^{1} \mathrm{H}$ NMR of 4-THF-d $\boldsymbol{d}_{\mathbf{8}}\left(\mathrm{THF}-d_{8}\right)$. (a) The full spectrum, and expansions of key regions: (b) $\delta 180-60$, (c) $\delta 20-5$, (d) $\delta 10--10$, and (e) $\delta-10--50$ are shown. 


\section{Crystallographic Report of 1-PMe}

General information: Crystals were grown from pentane under nitrogen atmosphere. The crystals appeared to be extremely sensitive to air and moisture. All crystals manipulations including mounting were carried out under nitrogen atmosphere in a glove bag. To further minimize crystal decomposition, nitrogen atmosphere was pre-cooled to about $15{ }^{\circ} \mathrm{C}$. A black block $\left(0.48 \times 0.24 \times 0.18 \mathrm{~mm}^{3}\right)$ was mounted on a Dual-Thickness MicroMount ${ }^{\mathrm{tm}}($ MiTeGen) with $20 \mu \mathrm{m}$ sample aperture with Fluorolube ${ }^{\mathrm{TM}}$ oil. The diffraction data were measured at $100 \mathrm{~K}$ on a Bruker D8 VENTURE with PHOTON 100 CMOS detector system equipped with a Motarget X-ray tube $(\lambda=0.71073 \AA)$. Data were collected using $\phi$ and $\omega$ scans to survey a hemisphere of reciprocal space. Data reduction and integration were performed with the Bruker APEX3 software package (Bruker AXS, version 2015.5-2, 2015). Data were scaled and corrected for absorption effects using the multi-scan procedure as implemented in SADABS (Bruker AXS, version 2014/5, 2015, part of Bruker APEX3 software package). The structure was solved by SHELXT (Version 2014/5: Sheldrick, G. M. Acta Cryst. 2015, A71, 3-8) and refined by a full-matrix least-squares procedure using Bruker SHELXTL (version 6.14) and OLEX2 (O. V. Dolomanov, L. J. Bourhis, R. J. Gildea, J. A. K. Howard and H. Puschmann. J. Appl. Cryst. (2009). 42, 339-341) software packages (XL refinement program version 2014/7, Sheldrick, G. M. Acta Cryst. 2008, A64, 112-122; Sheldrick, G. M. Acta Cryst. 2015, C71, 3-8). Crystallographic data and details of the data collection and structure refinement are listed in Table S-2.

Specific details for structure refinement: Significant elongation of essentially all thermal ellipsoids was observed (Figure S-21). This was modeled as a whole body disorder of two the same complexes sitting at almost the same location (see Figure S-22 for overlaid parts, and Figures S-23 and S-24 for separate parts). The refined ratio of two molecules is 60/40. All atoms were refined with anisotropic thermal parameters utilizing constraints on thermal parameters (RIGU, SIMU, EADP). While geometric restraints were initially used to support the refinement, geometric parameters were not restrained during final refinement cycles except soft SADI restraints were imposed on all $i$-Pr groups. Hydrogen atoms were included in idealized positions for structure factor calculations. After modelling the disorder, the data to parameters ratio is about 9 . All structures are drawn with thermal ellipsoids at $40 \%$ probability level. 


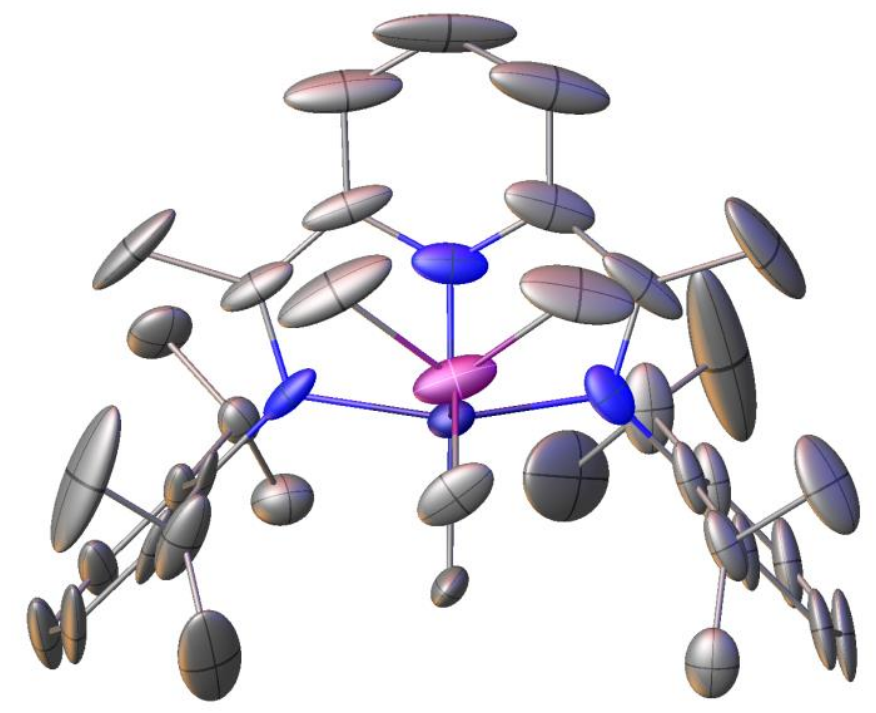

Figure S-21. Molecular structure of 1-PMe 3 before disorder modelling.

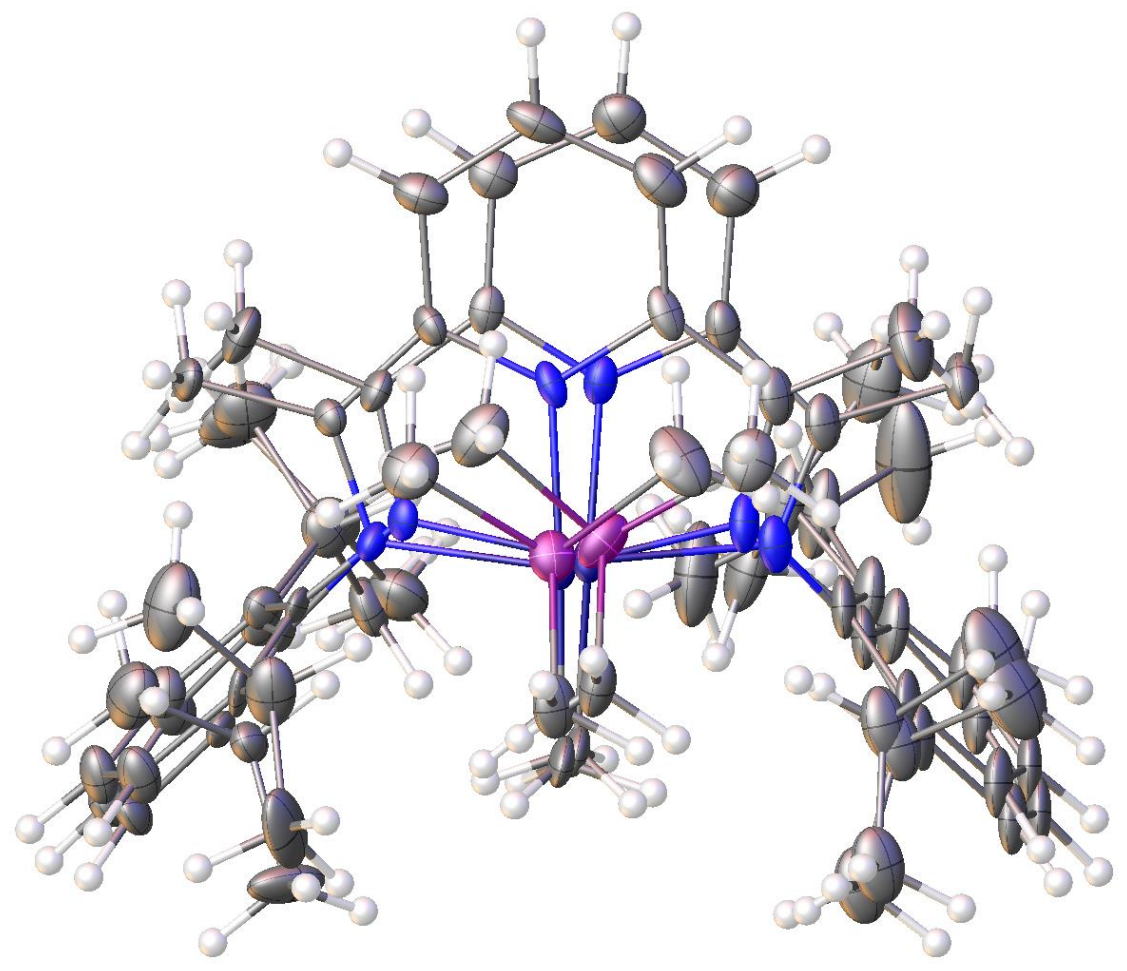

Figure S-22. Molecular structure of 1-PMe 3 after modelling as whole-body disorder of two molecules sitting at similar location. 


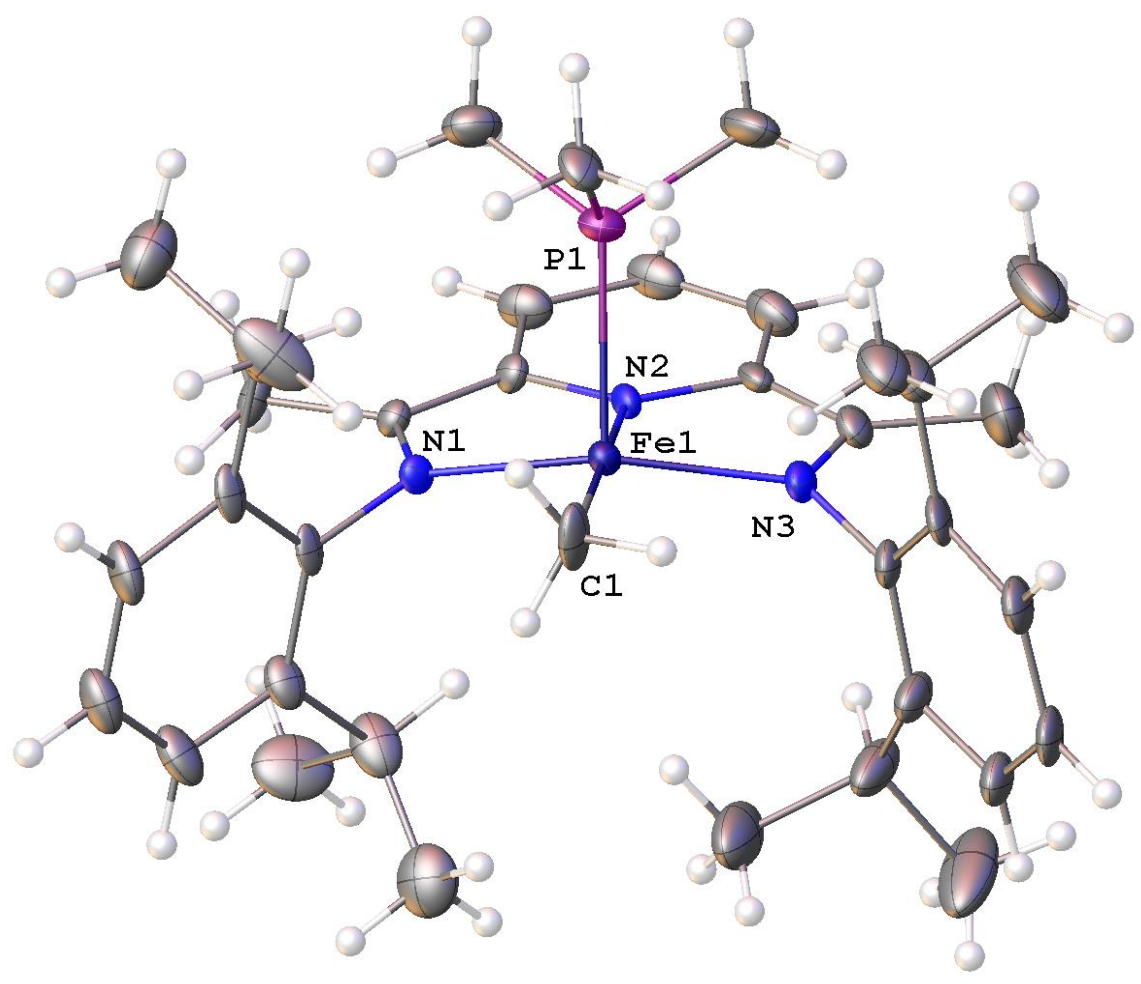

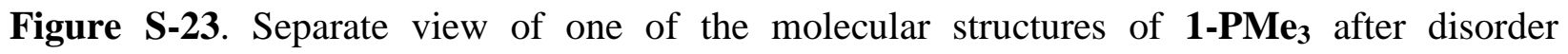
modelling. The other molecular structure of $\mathbf{1 - P M e} 3$ is shown in Figure S-24.

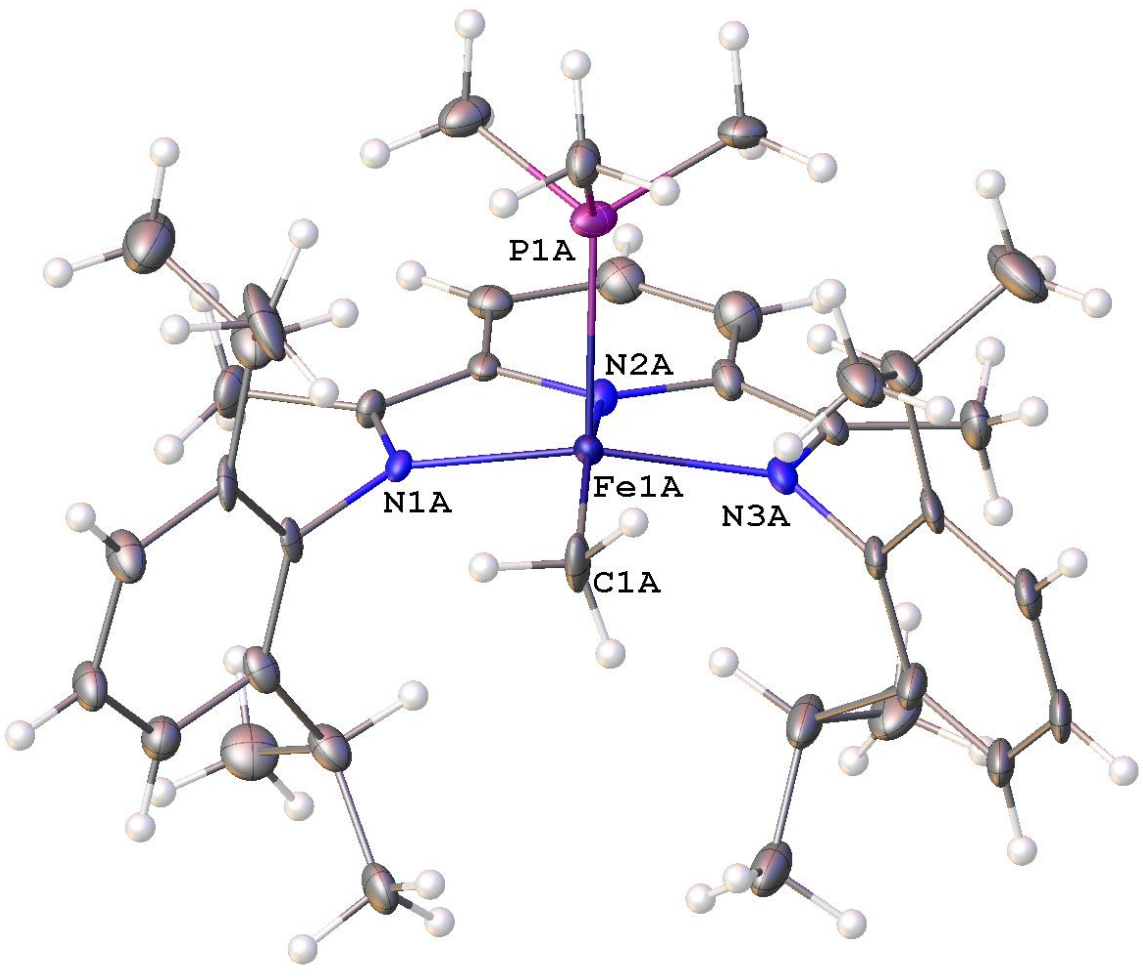


Figure S-24. Separate view of the other molecular structure of 1-PMe 3 after disorder modelling. The other molecular structure of 1-PMe $\mathbf{P M}_{3}$ is shown in Figure S-23.

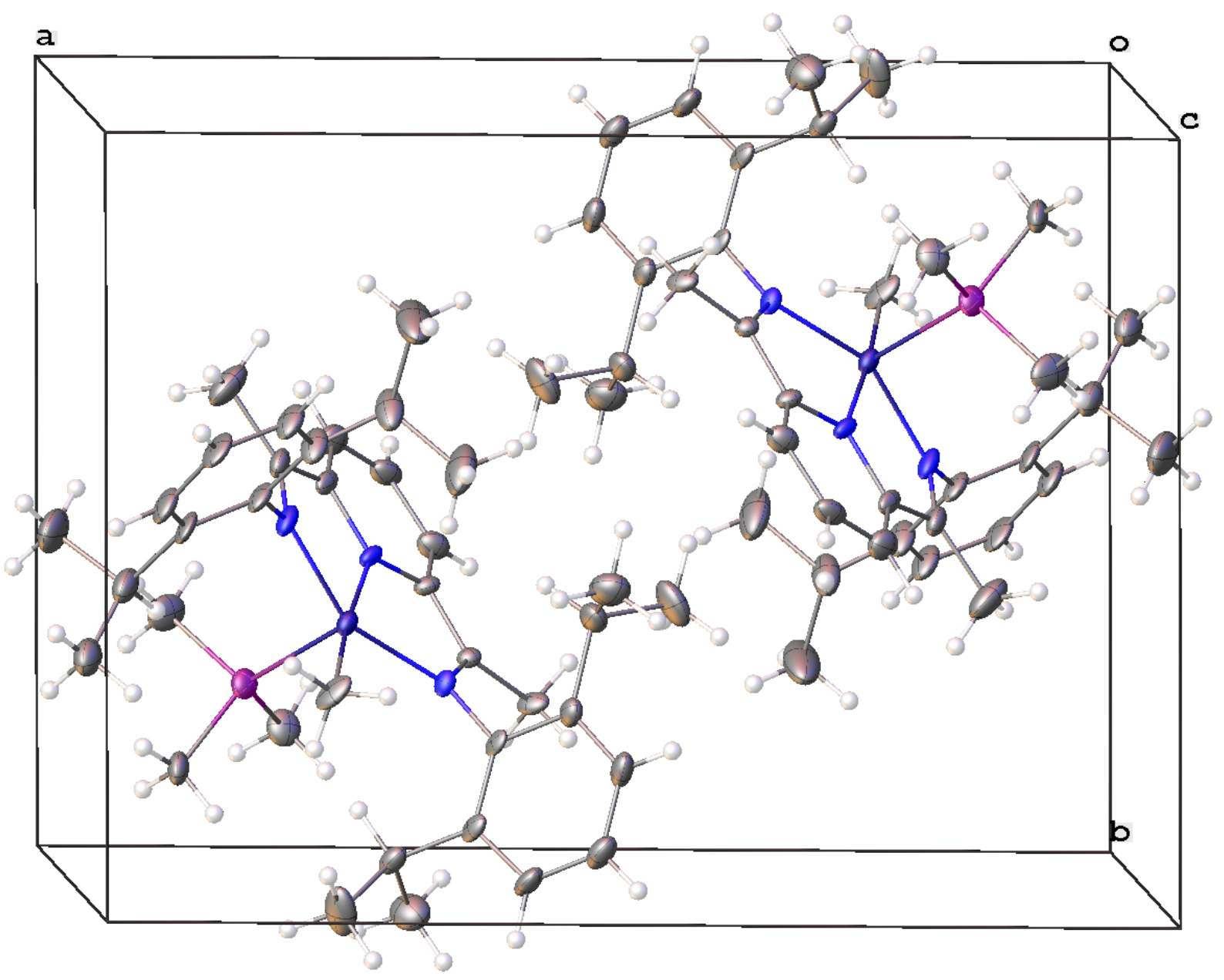

Figure S-25. Unit cell of 1-PMe . 
Table S-2. Crystal data and structure refinement for 1-PMe 3 .

Identification code

Empirical formula

Formula weight

Temperature/K

Crystal system

Space group

$\mathrm{a} / \AA$

$\mathrm{b} / \AA$

c/Å

$\alpha /{ }^{\circ}$

$\beta /{ }^{\circ}$

$\gamma /{ }^{\circ}$

Volume $/ \AA^{3}$

Z

$\rho_{\text {calcg }} / \mathrm{cm}^{3}$

$\mu / \mathrm{mm}^{-1}$

$\mathrm{F}(000)$

Crystal size $/ \mathrm{mm}^{3}$

Radiation

$2 \Theta$ range for data collection/ ${ }^{\circ}$

Index ranges

Reflections collected

Independent reflections

Data/restraints/parameters

Goodness-of-fit on $\mathrm{F}^{2}$

Final $\mathrm{R}$ indexes $[\mathrm{I}>=2 \sigma(\mathrm{I})]$

Final $\mathrm{R}$ indexes [all data]

Largest diff. peak/hole / e $\AA^{-3}$
1-PMe 3

$\mathrm{C}_{37} \mathrm{H}_{55} \mathrm{FeN}_{3} \mathrm{P}$

628.66

100(2)

monoclinic

$P 2_{1} / c$

17.2088(12)

$12.6102(8)$

$16.3707(11)$

90

100.567(2)

90

3492.3(4)

4

1.196

0.506

1356.0

$0.48 \times 0.24 \times 0.18$

$\operatorname{MoK} \alpha(\lambda=0.71073)$

4.516 to 50.106

$-20 \leq \mathrm{h} \leq 20,-14 \leq \mathrm{k} \leq 14,-19 \leq 1 \leq 19$

30607

$6181\left[\mathrm{R}_{\text {int }}=0.0661\right]$

$6181 / 2190 / 696$

1.045

$\mathrm{R}_{1}=0.0751, \mathrm{wR}_{2}=0.1483$

$\mathrm{R}_{1}=0.0933, \mathrm{wR}_{2}=0.1560$

$0.50 /-0.59$

$\mathrm{R}_{\mathrm{int}}=\Sigma\left|\mathrm{F}_{\mathrm{o}}^{2}-\left\langle\mathrm{F}_{\mathrm{o}}^{2}\right\rangle\right| / \Sigma\left|\mathrm{F}_{\mathrm{o}}{ }^{2}\right|$

$\mathrm{R} 1=\Sigma|| \mathrm{F}_{\mathrm{o}}|-| \mathrm{F}_{\mathrm{c}} \| / \Sigma\left|\mathrm{F}_{\mathrm{o}}\right|$

$\mathrm{wR} 2=\left[\Sigma\left[\mathrm{w}\left(\mathrm{F}_{\mathrm{o}}{ }^{2}-\mathrm{F}_{\mathrm{c}}{ }^{2}\right)^{2}\right] / \Sigma\left[\mathrm{w}\left(\mathrm{F}_{\mathrm{o}}{ }^{2}\right)^{2}\right]\right]^{1 / 2}$

Goodness-of-fit $=\left[\Sigma\left[\mathrm{w}\left(\mathrm{F}_{\mathrm{o}}{ }^{2-} \mathrm{F}_{\mathrm{c}}{ }^{2}\right)^{2}\right] /(\mathrm{n}-\mathrm{p})^{1 / 2}\right.$

$\mathrm{n}$ : number of independent reflections; $\mathrm{p}$ : number of refined parameters 
Table S-3. Fractional Atomic Coordinates $\left(\times 10^{4}\right)$ and Equivalent Isotropic Displacement Parameters $\left(\AA^{2} \times 10^{3}\right)$ for 1-PMe $\mathbf{U}_{\text {eq }}$ is defined as $1 / 3$ of of the trace of the orthogonalised $U_{\text {IJ }}$ tensor.

\begin{tabular}{|c|c|c|c|c|}
\hline Atom & $x$ & $y$ & $z$ & $\mathbf{U}(\mathbf{e q})$ \\
\hline $\mathrm{P} 1$ & $8398(2)$ & $7444(3)$ & $5111(3)$ & $22.8(7)$ \\
\hline $\mathrm{C} 2$ & $9048(7)$ & $8470(10)$ & $5635(11)$ & $30(3)$ \\
\hline C3 & 7971 ( 5) & $8093(8)$ & $4133(5)$ & 31.7 (19) \\
\hline $\mathrm{C} 4$ & $9099(6)$ & $6587(8)$ & $4723(6)$ & $40(2)$ \\
\hline Fe1 & 7485 (3) & $6605(5)$ & $5811(4)$ & $15.7(6)$ \\
\hline $\mathrm{C} 1$ & $7680(30)$ & $7380(30)$ & $6900(20)$ & $31(3)$ \\
\hline N1 & $6542(5)$ & $7405(8)$ & $5303(6)$ & $15.6(19)$ \\
\hline N2 & $7197(5)$ & $5847(6)$ & 4817 ( 5) & $16.5(16)$ \\
\hline N3 & $8060(7)$ & $5280(9)$ & $6103(6)$ & $23(2)$ \\
\hline $\mathrm{C} 5$ & $7582(5)$ & $10201(9)$ & $6312(6)$ & $47(3)$ \\
\hline C6 & $6841(5)$ & $10352(7)$ & 4841 ( 6 ) & $44(2)$ \\
\hline $\mathrm{C} 7$ & $7050(5)$ & $9621(6)$ & $5590(4)$ & $24.1(17)$ \\
\hline $\mathrm{C} 8$ & $6311(5)$ & $9186(8)$ & $5844(5)$ & $20.7(18)$ \\
\hline C9 & $5825(6)$ & $9822(7)$ & $6225(6)$ & $28.1(18)$ \\
\hline $\mathrm{C} 10$ & $5163(5)$ & $9423(8)$ & $6475(6)$ & $31(2)$ \\
\hline $\mathrm{C} 11$ & $4963(6)$ & $8383(9)$ & $6358(7)$ & $29(2)$ \\
\hline $\mathrm{C} 12$ & $5411(7)$ & $7699(11)$ & $5974(8)$ & $22.7(16)$ \\
\hline $\mathrm{C} 13$ & $6093(6)$ & $8114(9)$ & $5714(6)$ & $16.9(15)$ \\
\hline $\mathrm{C} 14$ & $5193(15)$ & $6509(18)$ & $5857(13)$ & $32(3)$ \\
\hline $\mathrm{C} 15$ & 5095 (19) & $6068(18)$ & $6698(15)$ & $43(5)$ \\
\hline $\mathrm{C} 16$ & $4411(15)$ & $6380(30)$ & $5234(16)$ & $47(6)$ \\
\hline $\mathrm{C} 17$ & $5632(5)$ & $7782(7)$ & 3941 ( 5 ) & $22.4(18)$ \\
\hline C18 & $6264(5)$ & $7154(7)$ & 4497 ( 5) & $14.6(18)$ \\
\hline C19 & $6636(5)$ & $6274(8)$ & $4196(5)$ & $16.3(17)$ \\
\hline $\mathrm{C} 20$ & $6525(5)$ & $5839(7)$ & 3402 ( 5 ) & $25.6(17)$ \\
\hline $\mathrm{C} 21$ & $6955(5)$ & $4935(8)$ & 3270 ( 5 ) & $27.0(18)$ \\
\hline $\mathrm{C} 22$ & 7489 (6) & $4461(8)$ & 3915 (6) & $29.6(18)$ \\
\hline $\mathrm{C} 23$ & 7601 ( 5) & $4927(7)$ & 4701 ( 5) & $19.6(16)$ \\
\hline $\mathrm{C} 24$ & $8088(5)$ & $4614(7)$ & $5461(6)$ & $23.3(14)$ \\
\hline $\mathrm{C} 25$ & $8571(6)$ & $3630(8)$ & $5501(7)$ & $48(2)$ \\
\hline $\mathrm{C} 26$ & $10023(16)$ & 6877 (19) & $7640(20)$ & $42(3)$ \\
\hline $\mathrm{C} 27$ & $10282(12)$ & $5370(20)$ & $6744(14)$ & $59(5)$ \\
\hline $\mathrm{C} 28$ & $9629(12)$ & 6026 (19) & 7040 (12) & $36(3)$ \\
\hline $\mathrm{C} 29$ & $9086(8)$ & $5256(14)$ & $7393(7)$ & $28.3(19)$ \\
\hline $\mathrm{C} 30$ & $9329(9)$ & $4899(14)$ & $8194(7)$ & $34(2)$ \\
\hline C31 & $8869(8)$ & $4161(12)$ & $8545(6)$ & $35(2)$ \\
\hline C32 & $8158(8)$ & $3838(12)$ & 8088 ( 6) & $34(2)$ \\
\hline $\mathrm{C} 33$ & $7883(7)$ & $4217(11)$ & $7295(6)$ & $33.8(19)$ \\
\hline
\end{tabular}




\begin{tabular}{|c|c|c|c|c|}
\hline C34 & $8366(8)$ & $4909(11)$ & $6934(6)$ & $25.8(16)$ \\
\hline $\mathrm{C} 35$ & $7162(6)$ & $3778(8)$ & $7009(8)$ & $46(2)$ \\
\hline $\mathrm{C} 36$ & $6947(6)$ & $2645(8)$ & $7253(10)$ & $87(4)$ \\
\hline C37 & $6510(5)$ & $4520(9)$ & $7176(7)$ & $62(3)$ \\
\hline P1A & 8595 (3) & 7105 (5) & $5148(4)$ & $26.4(12)$ \\
\hline $\mathrm{C} 3 \mathrm{~A}$ & $8247(8)$ & $7583(12)$ & $4090(8)$ & $34(3)$ \\
\hline $\mathrm{C} 2 \mathrm{~A}$ & $9241(11)$ & 8187 (15) & $5607(15)$ & $28(4)$ \\
\hline $\mathrm{C} 4 \mathrm{~A}$ & $9302(8)$ & $6132(12)$ & $4937(8)$ & $31(3)$ \\
\hline $\mathrm{Fe} 1 \mathrm{~A}$ & $7612(5)$ & $6434(7)$ & $5834(7)$ & $15.7(6)$ \\
\hline $\mathrm{C} 1 \mathrm{~A}$ & $7636(18)$ & $7171(18)$ & $6933(15)$ & $31(3)$ \\
\hline N1A & $6699(8)$ & $7193(12)$ & $5220(9)$ & $14(2)$ \\
\hline $\mathrm{N} 2 \mathrm{~A}$ & $7355(7)$ & $5520(11)$ & $4942(8)$ & $19(2)$ \\
\hline N3A & $8180(10)$ & $5171(13)$ & $6316(10)$ & $22(3)$ \\
\hline C5A & $7756(7)$ & $10192(11)$ & $5848(11)$ & $53(4)$ \\
\hline C6A & $7006(9)$ & $9859(13)$ & $4382(9)$ & $55(4)$ \\
\hline C7A & $7226(8)$ & $9414(11)$ & $5269(8)$ & $39(3)$ \\
\hline $\mathrm{C} 8 \mathrm{~A}$ & $6489(8)$ & $9055(11)$ & $5584(9)$ & $24(3)$ \\
\hline C9A & $6031(8)$ & $9833(12)$ & $5888(10)$ & $30(3)$ \\
\hline C10A & $5320(10)$ & $9573(11)$ & $6143(10)$ & $28(3)$ \\
\hline C11A & $5110(10)$ & $8530(13)$ & $6100(9)$ & $26(3)$ \\
\hline C12A & $5543(12)$ & $7739(17)$ & $5804(13)$ & $22.7(16)$ \\
\hline C13A & $6222(10)$ & 8012 (15) & $5520(10)$ & $16.9(15)$ \\
\hline C14A & $5220(20)$ & $6650(30)$ & $5847(18)$ & $28(4)$ \\
\hline $\mathrm{C} 15 \mathrm{~A}$ & $5020(30)$ & $6300(30)$ & $6680(20)$ & $30(5)$ \\
\hline C16A & $4540(20)$ & $6390(40)$ & $5130(30)$ & $39(6)$ \\
\hline C17A & $5849(7)$ & $7335(12)$ & $3801(8)$ & $30(3)$ \\
\hline C18A & $6462(8)$ & 6818 (13) & $4437(9)$ & $18(3)$ \\
\hline C19A & $6836(7)$ & $5881(11)$ & $4259(8)$ & $17(2)$ \\
\hline $\mathrm{C} 20 \mathrm{~A}$ & $6738(8)$ & $5304(13)$ & $3513(8)$ & $23(2)$ \\
\hline $\mathrm{C} 21 \mathrm{~A}$ & $7160(8)$ & $4345(12)$ & $3537(10)$ & $29(3)$ \\
\hline $\mathrm{C} 22 \mathrm{~A}$ & $7661(7)$ & $3975(12)$ & $4243(8)$ & $32(3)$ \\
\hline $\mathrm{C} 23 \mathrm{~A}$ & $7748(8)$ & $4577(11)$ & $4977(9)$ & $22(2)$ \\
\hline $\mathrm{C} 24 \mathrm{~A}$ & $8221(8)$ & $4384(11)$ & $5779(8)$ & $23.3(14)$ \\
\hline $\mathrm{C} 25 \mathrm{~A}$ & $8649(7)$ & $3363(10)$ & $5962(8)$ & $30(3)$ \\
\hline $\mathrm{C} 26 \mathrm{~A}$ & $9910(30)$ & $7000(30)$ & $7750(30)$ & $42(3)$ \\
\hline $\mathrm{C} 27 \mathrm{~A}$ & $10410(20)$ & $5430(40)$ & $7020(20)$ & $59(5)$ \\
\hline $\mathrm{C} 28 \mathrm{~A}$ & $9682(19)$ & $6010(30)$ & $7210(20)$ & $36(3)$ \\
\hline C29A & $9170(13)$ & $5310(20)$ & $7626(12)$ & $28.3(19)$ \\
\hline C30A & $9402(15)$ & $4950(20)$ & $8444(12)$ & $34(2)$ \\
\hline C31A & 8962 (13) & 4303 (19) & $8820(11)$ & $35(2)$ \\
\hline C32A & $8246(13)$ & 3931 (19) & $8377(10)$ & $34(2)$ \\
\hline $\mathrm{C} 33 \mathrm{~A}$ & 7978 (12) & 4176 (19) & $7556(11)$ & $33.8(19)$ \\
\hline
\end{tabular}




$\begin{array}{lrrrr}\text { C34A } & 8446(12) & 4893(18) & 7174(10) & 25.8(16) \\ \text { C35A } & 7040(11) & 3926(13) & 6830(12) & 46(2) \\ \text { C36A } & 7069(8) & 2791(10) & 6515(9) & 49(3) \\ \text { C37A } & 6463(7) & 3990(13) & 7438(9) & 46(3)\end{array}$

Table S-4. Anisotropic Displacement Parameters $\left(\AA^{2} \times 10^{3}\right)$ for 1-PMe 3 . The Anisotropic displacement factor exponent takes the form: $-2 \pi^{2}\left[\mathrm{~h}^{2} \mathrm{a} * 2 \mathrm{U}_{11}+2 \mathrm{hka} * \mathrm{~b} * \mathrm{U}_{12}+\ldots\right]$.

\begin{tabular}{|c|c|c|c|c|c|c|}
\hline Atom & $\mathbf{U}_{11}$ & $\mathbf{U}_{22}$ & $\mathbf{U}_{33}$ & $\mathbf{U}_{23}$ & $\mathbf{U}_{13}$ & $\mathbf{U}_{12}$ \\
\hline $\mathrm{P} 1$ & $19.9(17)$ & $25.5(19)$ & $25.5(13)$ & $4.5(13)$ & $10.9(12)$ & $-0.3(11)$ \\
\hline $\mathrm{C} 2$ & $13(5)$ & $31(6)$ & $46(5)$ & $7(5)$ & $7(4)$ & $2(4)$ \\
\hline $\mathrm{C} 3$ & $33(5)$ & $38(5)$ & $27(4)$ & $6(3)$ & $12(3)$ & $-3(4)$ \\
\hline $\mathrm{C} 4$ & $43(5)$ & $37(5)$ & $50(5)$ & $6(4)$ & $35(4)$ & $5(4)$ \\
\hline $\mathrm{Fe} 1$ & $13.3(16)$ & $17.5(17)$ & $17.2(4)$ & $4(1)$ & $5.1(10)$ & $3.9(9)$ \\
\hline $\mathrm{C} 1$ & $33(4)$ & $33(8)$ & $24(3)$ & $4(4)$ & $-7(2)$ & $20(6)$ \\
\hline N1 & $13(3)$ & $20(4)$ & $13(3)$ & $3(3)$ & $0(2)$ & $4(3)$ \\
\hline $\mathrm{N} 2$ & $16(3)$ & $12(3)$ & $24(3)$ & $3(2)$ & $10(2)$ & $5(2)$ \\
\hline N3 & $14(4)$ & $25(3)$ & $31(4)$ & 12 ( 3 ) & $7(3)$ & $8(3)$ \\
\hline $\mathrm{C} 5$ & $27(4)$ & $72(7)$ & $39(5)$ & $-32(5)$ & $0(3)$ & $-9(4)$ \\
\hline C6 & $43(5)$ & $36(5)$ & $49(5)$ & $10(4)$ & $-4(4)$ & $1(4)$ \\
\hline $\mathrm{C} 7$ & $21(4)$ & $23(4)$ & $27(4)$ & $-7(3)$ & $-1(3)$ & $7(3)$ \\
\hline $\mathrm{C} 8$ & $15(4)$ & $22(3)$ & $20(4)$ & $-5(3)$ & $-8(3)$ & $9(3)$ \\
\hline C9 & $22(4)$ & $24(4)$ & $36(5)$ & $-9(3)$ & $1(3)$ & $11(3)$ \\
\hline $\mathrm{C} 10$ & $25(4)$ & $32(4)$ & $36(5)$ & $-10(4)$ & $1(3)$ & $11(3)$ \\
\hline C11 & $15(4)$ & $36(4)$ & $35(6)$ & $-10(4)$ & $0(3)$ & $5(3)$ \\
\hline $\mathrm{C} 12$ & $14(3)$ & $24(2)$ & $27(4)$ & $-4(2)$ & $-2(2)$ & $2(2)$ \\
\hline $\mathrm{C} 13$ & 12 (3) & $20(2)$ & $16(4)$ & $-2(2)$ & $-7(2)$ & $7.6(19)$ \\
\hline $\mathrm{C} 14$ & $21(6)$ & $26(6)$ & $48(6)$ & $-11(4)$ & $-1(4)$ & $-4(4)$ \\
\hline $\mathrm{C} 15$ & $47(11)$ & $32(9)$ & $48(6)$ & $-11(5)$ & $2(5)$ & $0(8)$ \\
\hline C16 & 32 ( 8 ) & $60(9)$ & $45(8)$ & $-10(7)$ & $-3(6)$ & $-22(7)$ \\
\hline $\mathrm{C} 17$ & $27(4)$ & $14(4)$ & $22(4)$ & $4(3)$ & $-6(3)$ & $5(3)$ \\
\hline C18 & $17(4)$ & $11(4)$ & $16(3)$ & $2(3)$ & $3(2)$ & $1(3)$ \\
\hline C19 & $20(4)$ & $11(4)$ & $19(3)$ & $2(3)$ & $5(3)$ & $3(3)$ \\
\hline $\mathrm{C} 20$ & $30(4)$ & $23(4)$ & $25(3)$ & $-6(3)$ & $11(3)$ & $-4(3)$ \\
\hline C21 & 31 ( 4) & 21 ( 4 ) & $33(4)$ & $-11(3)$ & $16(3)$ & $-6(3)$ \\
\hline $\mathrm{C} 22$ & $27(4)$ & $26(4)$ & $40(4)$ & $-5(3)$ & $17(3)$ & $1(3)$ \\
\hline $\mathrm{C} 23$ & $16(3)$ & $16(4)$ & 31 (3) & $6(3)$ & 15 (3) & $3(3)$ \\
\hline $\mathrm{C} 24$ & $13(3)$ & $23(3)$ & $35(4)$ & $7(2)$ & $8(3)$ & $5(2)$ \\
\hline $\mathrm{C} 25$ & $43(5)$ & $44(5)$ & $61(6)$ & $12(4)$ & $21(5)$ & $25(4)$ \\
\hline C26 & $24(7)$ & $38(5)$ & $67(8)$ & $9(4)$ & $16(5)$ & $3(4)$ \\
\hline $\mathrm{C} 27$ & $31(7)$ & $53(5)$ & 102 (15) & $3(9)$ & $32(8)$ & $9(5)$ \\
\hline
\end{tabular}




\begin{tabular}{|c|c|c|c|c|c|c|}
\hline $\mathrm{C} 28$ & $21(3)$ & $33(2)$ & $57(7)$ & $11(5)$ & $15(4)$ & $7(2)$ \\
\hline C29 & $14(3)$ & $27(3)$ & $44(5)$ & $12(4)$ & $5(3)$ & $13.0(19)$ \\
\hline $\mathrm{C} 30$ & $19(3)$ & $34(3)$ & $47(6)$ & $14(5)$ & $4(4)$ & $15(2)$ \\
\hline C31 & $24(3)$ & 31 ( 4) & $47(6)$ & $18(5)$ & $3(4)$ & $18(2)$ \\
\hline C32 & $22(3)$ & $32(3)$ & $47(5)$ & $21(5)$ & $6(4)$ & $13(2)$ \\
\hline $\mathrm{C} 33$ & $21(3)$ & $33(3)$ & $47(5)$ & $24(4)$ & $5(3)$ & $8(2)$ \\
\hline C34 & $18(3)$ & $23(2)$ & $37(4)$ & $13(3)$ & $6(3)$ & $12.0(19)$ \\
\hline $\mathrm{C} 35$ & $22(3)$ & $45(4)$ & $68(5)$ & $37(3)$ & $2(3)$ & $2(3)$ \\
\hline C36 & $38(5)$ & $47(5)$ & $170(13)$ & $55(6)$ & $8(6)$ & $-4(4)$ \\
\hline C37 & $28(4)$ & $66(6)$ & $93(8)$ & $44(6)$ & $11(5)$ & $15(4)$ \\
\hline P1A & $16(2)$ & $39(3)$ & $22.8(17)$ & $8(2)$ & $1.2(18)$ & $-6.5(19)$ \\
\hline $\mathrm{C} 3 \mathrm{~A}$ & $26(6)$ & $44(8)$ & $32(5)$ & $12(5)$ & $4(4)$ & $-9(5)$ \\
\hline $\mathrm{C} 2 \mathrm{~A}$ & $18(8)$ & $28(8)$ & $36(7)$ & $10(6)$ & $-2(6)$ & $3(6)$ \\
\hline $\mathrm{C} 4 \mathrm{~A}$ & $23(6)$ & $41(7)$ & $32(6)$ & $10(5)$ & $13(5)$ & $-1(5)$ \\
\hline $\mathrm{Fe} 1 \mathrm{~A}$ & $13.3(16)$ & $17.5(17)$ & $17.2(4)$ & $4(1)$ & $5.1(10)$ & $3.9(9)$ \\
\hline $\mathrm{C} 1 \mathrm{~A}$ & $33(4)$ & $33(8)$ & $24(3)$ & $4(4)$ & $-7(2)$ & $20(6)$ \\
\hline N1A & $13(4)$ & $11(5)$ & $18(4)$ & $6(3)$ & $2(3)$ & $0(3)$ \\
\hline $\mathrm{N} 2 \mathrm{~A}$ & $13(4)$ & $19(5)$ & $24(4)$ & $6(3)$ & $2(3)$ & $1(3)$ \\
\hline N3A & $12(5)$ & $20(4)$ & $34(5)$ & $8(3)$ & $6(4)$ & $4(3)$ \\
\hline C5A & $16(5)$ & $37(7)$ & $100(10)$ & $-5(7)$ & $-4(6)$ & $10(5)$ \\
\hline C6A & $39(7)$ & $54(9)$ & $71(7)$ & $28(6)$ & $5(6)$ & $0(7)$ \\
\hline C7A & $25(5)$ & $28(6)$ & $60(7)$ & $7(5)$ & $0(4)$ & $-5(4)$ \\
\hline C8A & $14(5)$ & $17(4)$ & $36(7)$ & $5(4)$ & $-11(4)$ & $6(3)$ \\
\hline C9A & $21(5)$ & $19(5)$ & $49(7)$ & $0(5)$ & $3(5)$ & $0(4)$ \\
\hline C10A & $21(6)$ & $17(5)$ & $44(8)$ & $4(4)$ & $4(5)$ & $3(4)$ \\
\hline C11A & $24(6)$ & $24(4)$ & $29(7)$ & $4(4)$ & $3(4)$ & $1(4)$ \\
\hline C12A & $14(3)$ & $24(2)$ & $27(4)$ & $-4(2)$ & $-2(2)$ & $2(2)$ \\
\hline C13A & $12(3)$ & $20(2)$ & $16(4)$ & $-2(2)$ & $-7(2)$ & $7.6(19)$ \\
\hline C14A & $18(7)$ & $26(6)$ & $41(7)$ & $-3(5)$ & $5(5)$ & $1(5)$ \\
\hline C15A & $23(8)$ & $22(12)$ & $46(8)$ & $1(7)$ & $7(6)$ & $9(8)$ \\
\hline C16A & $30(10)$ & $37(11)$ & $49(9)$ & $-4(7)$ & $1(8)$ & $-12(8)$ \\
\hline C17A & $24(6)$ & $35(8)$ & $27(6)$ & $10(5)$ & $-7(5)$ & $5(5)$ \\
\hline C18A & $16(5)$ & $20(6)$ & $15(4)$ & $5(4)$ & $-1(3)$ & $3(4)$ \\
\hline C19A & $10(5)$ & $19(5)$ & $22(4)$ & $7(4)$ & $4(3)$ & $-1(4)$ \\
\hline C20A & $20(6)$ & $25(6)$ & $26(5)$ & $1(4)$ & $9(4)$ & $-4(4)$ \\
\hline C21A & $27(6)$ & $26(6)$ & $34(6)$ & $-2(4)$ & $5(4)$ & $-6(5)$ \\
\hline $\mathrm{C} 22 \mathrm{~A}$ & $30(6)$ & $33(6)$ & $34(5)$ & $-2(4)$ & $4(4)$ & $1(5)$ \\
\hline C23A & $15(5)$ & $18(5)$ & $33(5)$ & $4(3)$ & $4(4)$ & $3(4)$ \\
\hline C24A & $13(3)$ & $23(3)$ & $35(4)$ & $7(2)$ & $8(3)$ & $5(2)$ \\
\hline $\mathrm{C} 25 \mathrm{~A}$ & $29(6)$ & $27(5)$ & $35(6)$ & $6(5)$ & $6(5)$ & $15(5)$ \\
\hline C26A & $24(7)$ & $38(5)$ & $67(8)$ & $9(4)$ & $16(5)$ & $3(4)$ \\
\hline C27A & $31(7)$ & $53(5)$ & $102(15)$ & $3(9)$ & $32(8)$ & $9(5)$ \\
\hline
\end{tabular}




$\begin{array}{lllllrr}\text { C28A } & 21(3) & 33(2) & 57(7) & 11(5) & 15(4) & 7(2) \\ \text { C29A } & 14(3) & 27(3) & 44(5) & 12(4) & 5(3) & 13.0(19) \\ \text { C30A } & 19(3) & 34(3) & 47(6) & 14(5) & 4(4) & 15(2) \\ \text { C31A } & 24(3) & 31(4) & 47(6) & 18(5) & 3(4) & 18(2) \\ \text { C32A } & 22(3) & 32(3) & 47(5) & 21(5) & 6(4) & 13(2) \\ \text { C33A } & 21(3) & 33(3) & 47(5) & 24(4) & 5(3) & 8(2) \\ \text { C34A } & 18(3) & 23(2) & 37(4) & 13(3) & 6(3) & 12.0(19) \\ \text { C35A } & 22(3) & 45(4) & 68(5) & 37(3) & 2(3) & 2(3) \\ \text { C36A } & 39(7) & 40(6) & 66(8) & 25(5) & 7(6) & -9(5) \\ \text { C37A } & 27(5) & 49(8) & 58(7) & 33(6) & -2(5) & 0(5)\end{array}$

Table S-5. Bond Lengths for 1-PMe

\begin{tabular}{llrlr} 
Atom Atom & Length/A & Atom Atom & \multicolumn{1}{c}{ Length/A } \\
P1 & C2 & $1.819(13)$ & P1A C3A & $1.827(13)$ \\
P1 & C3 & $1.828(9)$ & P1A C2A & $1.83(2)$ \\
P1 & C4 & $1.818(9)$ & P1A C4A & $1.805(14)$ \\
P1 & Fe1 & $2.359(8)$ & P1A Fe1A & $2.353(12)$ \\
Fe1 & C1 & $2.00(4)$ & Fe1A C1A & $2.02(2)$ \\
Fe1 & N1 & $1.962(9)$ & Fe1A N1A & $1.954(15)$ \\
Fe1 & N2 & $1.874(10)$ & Fe1A N2A & $1.849(17)$ \\
Fe1 & N3 & $1.955(10)$ & Fe1A N3A & $1.957(16)$ \\
N1 & C13 & $1.428(10)$ & N1A C13A & $1.459(16)$ \\
N1 & C18 & $1.356(11)$ & N1A C18A & $1.357(19)$ \\
N2 & C19 & $1.376(10)$ & N2A C19A & $1.375(16)$ \\
N2 & C23 & $1.383(11)$ & N2A C23A & $1.364(17)$ \\
N3 & C24 & $1.354(13)$ & N3A C24A & $1.34(2)$ \\
N3 & C34 & $1.443(10)$ & N3A C34A & $1.439(17)$ \\
C5 & C7 & $1.542(8)$ & C5A C7A & $1.541(12)$ \\
C6 & C7 & $1.523(9)$ & C6A C7A & $1.538(12)$ \\
C7 & C8 & $1.511(11)$ & C7A C8A & $1.524(18)$ \\
C8 & C9 & $1.388(12)$ & C8A C9A & $1.405(19)$ \\
C8 & C13 & $1.407(13)$ & C8A C13A & $1.39(2)$ \\
C9 & C10 & $1.374(13)$ & C9A C10A & $1.402(19)$ \\
C10 & C11 & $1.361(13)$ & C10AC11A & $1.36(2)$ \\
C11 & C12 & $1.382(13)$ & C11AC12A & $1.38(2)$ \\
C12 & C13 & $1.420(12)$ & C12AC13A & $1.378(19)$ \\
C12 & C14 & $1.55(2)$ & C14A & $1.49(3)$ \\
C14 & C15 & $1.522(11)$ & C16A & $1.529(13)$ \\
C14 & C16 & $1.540(11)$ & $1.537(14)$ \\
C17 & C18 & $1.508(10)$ & $1.490(16)$
\end{tabular}




$\begin{array}{lllllr}\text { C18 } & \text { C19 } & 1.414(11) & \text { C18AC19A } & 1.402(19) \\ \text { C19 } & \text { C20 } & 1.391(11) & \text { C19AC20A } & 1.40(2) \\ \text { C20 } & \text { C21 } & 1.398(12) & \text { C20AC21A } & 1.41(2) \\ \text { C21 } & \text { C22 } & 1.401(13) & \text { C21AC22A } & 1.390(19) \\ \text { C22 } & \text { C23 } & 1.395(12) & \text { C22AC23A } & 1.405(17) \\ \text { C23 } & \text { C24 } & 1.423(11) & \text { C23AC24A } & 1.434(17) \\ \text { C24 } & \text { C25 } & 1.488(11) & \text { C24AC25A } & 1.486(16) \\ \text { C26 } & \text { C28 } & 1.528(11) & \text { C26AC28A } & 1.534(13) \\ \text { C27 } & \text { C28 } & 1.544(10) & \text { C27AC28A } & 1.534(13) \\ \text { C28 } & \text { C29 } & 1.53(2) & \text { C28AC29A } & 1.49(3) \\ \text { C29 } & \text { C30 } & 1.377(13) & \text { C29AC30A } & 1.40(2) \\ \text { C29 } & \text { C34 } & 1.397(14) & \text { C29AC34A } & 1.43(2) \\ \text { C30 } & \text { C31 } & 1.411(17) & \text { C30AC31A } & 1.34(3) \\ \text { C31 } & \text { C32 } & 1.373(14) & \text { C31AC32A } & 1.39(2) \\ \text { C32 } & \text { C33 } & 1.383(11) & \text { C32AC33A } & 1.374(19) \\ \text { C33 } & \text { C34 } & 1.408(16) & \text { C33AC34A } & 1.43(2) \\ \text { C33 } & \text { C35 } & 1.360(19) & \text { C33AC35A } & 1.85(3) \\ \text { C35 } & \text { C36 } & 1.547(11) & \text { C35AC36A } & 1.526(13) \\ \text { C35 } & \text { C37 } & 1.524(11) & \text { C35AC37A } & 1.531(13)\end{array}$

Table S-6. Bond Angles for 1-PMe .

\begin{tabular}{lllllr}
\multicolumn{1}{l}{ Atom Atom Atom } & \multicolumn{1}{c}{ Angle $^{\circ}$} & & Atom Atom Atom & \multicolumn{1}{c}{ Angle $^{\circ}$} \\
C2 & P1 & C3 & $102.0(6)$ & C3A P1A C2A & $102.2(9)$ \\
C2 & P1 & Fe1 & $119.9(6)$ & C3A P1A Fe1A & $115.5(5)$ \\
C3 & P1 & Fe1 & $115.5(4)$ & C2A P1A Fe1A & $120.5(8)$ \\
C4 & P1 & C2 & $101.4(5)$ & C4A P1A C3A & $99.1(7)$ \\
C4 & P1 & C3 & $98.3(5)$ & C4A P1A C2A & $101.9(7)$ \\
C4 & P1 & Fe1 & $116.5(4)$ & C4A P1A Fe1A & $114.5(5)$ \\
C1 & Fe1 & P1 & $101.1(14)$ & C1A Fe1A P1A & $111.1(9)$ \\
N1 & Fe1 & P1 & $97.8(4)$ & N1A Fe1A P1A & $99.2(6)$ \\
N1 & Fe1 & C1 & $96.8(13)$ & N1A Fe1A C1A & $96.6(9)$ \\
N2 & Fe1 & P1 & $84.3(4)$ & N1A Fe1A N3A & $154.3(9)$ \\
N2 & Fe1 & C1 & $174.2(15)$ & N2A Fe1A P1A & $86.4(6)$ \\
N2 & Fe1 & N1 & $80.1(4)$ & N2A Fe1A C1A & $162.5(11)$ \\
N2 & Fe1 & N3 & $79.5(4)$ & N2A Fe1A N1A & $81.1(7)$ \\
N3 & Fe1 & P1 & $98.6(4)$ & N2A Fe1A N3A & $80.9(7)$ \\
N3 & Fe1 & C1 & $101.7(12)$ & N3A Fe1A P1A & $97.8(6)$ \\
N3 & Fe1 & N1 & $152.3(6)$ & N3A Fe1A C1A & $95.1(9)$ \\
C13 & N1 & Fe1 & $126.8(7)$ & C13A N1A Fe1A & $128.5(11)$ \\
C18 & N1 & Fe1 & $114.2(6)$ & C18A N1A Fe1A & $112.9(10)$
\end{tabular}




\begin{tabular}{|c|c|c|c|c|c|}
\hline $\mathrm{C} 18$ & N1 & $\mathrm{C} 13$ & $118.7(8)$ & C18AN1A C13A & $118.4(13)$ \\
\hline C19 & $\mathrm{N} 2$ & $\mathrm{Fe} 1$ & $118.7(6)$ & C19AN2A Fe1A & $117.6(10)$ \\
\hline C19 & $\mathrm{N} 2$ & $\mathrm{C} 23$ & $121.9(8)$ & C23AN2A Fe1A & $117.7(9)$ \\
\hline $\mathrm{C} 23$ & $\mathrm{~N} 2$ & $\mathrm{Fe} 1$ & $119.2(6)$ & C23AN2A C19A & $124.4(13)$ \\
\hline $\mathrm{C} 24$ & N3 & $\mathrm{Fe} 1$ & $115.4(6)$ & C24AN3A Fe1A & $115.0(10)$ \\
\hline $\mathrm{C} 24$ & N3 & C34 & $118.1(9)$ & C24AN3A C34A & $114.5(14)$ \\
\hline C34 & N3 & $\mathrm{Fe} 1$ & $126.0(8)$ & C34AN3A Fe1A & $129.6(13)$ \\
\hline C6 & $\mathrm{C} 7$ & $\mathrm{C} 5$ & $110.5(7)$ & C6A C7A C5A & $111.4(12)$ \\
\hline $\mathrm{C} 8$ & $\mathrm{C} 7$ & $\mathrm{C} 5$ & $111.9(6)$ & C8A C7A C5A & $114.6(12)$ \\
\hline $\mathrm{C} 8$ & $\mathrm{C} 7$ & C6 & $110.8(6)$ & C8A C7A C6A & $110.6(10)$ \\
\hline C9 & $\mathrm{C} 8$ & $\mathrm{C} 7$ & $121.4(9)$ & C9A C8A C7A & $117.9(12)$ \\
\hline C9 & $\mathrm{C} 8$ & $\mathrm{C} 13$ & $117.2(8)$ & C13AC8A C7A & $122.7(12)$ \\
\hline $\mathrm{C} 13$ & $\mathrm{C} 8$ & $\mathrm{C} 7$ & $121.5(8)$ & C13AC8A C9A & $119.2(13)$ \\
\hline $\mathrm{C} 10$ & C9 & $\mathrm{C} 8$ & $121.6(9)$ & C10AC9A C8A & $121.2(13)$ \\
\hline $\mathrm{C} 11$ & $\mathrm{C} 10$ & $\mathrm{C} 9$ & $120.9(8)$ & C11AC10AC9A & $116.6(13)$ \\
\hline $\mathrm{C} 10$ & $\mathrm{C} 11$ & $\mathrm{C} 12$ & $121.1(9)$ & C10AC11AC12A & $124.0(14)$ \\
\hline $\mathrm{C} 11$ & $\mathrm{C} 12$ & $\mathrm{C} 13$ & $117.9(10)$ & C11A C12AC14A & $115.0(19)$ \\
\hline $\mathrm{C} 11$ & $\mathrm{C} 12$ & $\mathrm{C} 14$ & $121.1(12)$ & C13AC12AC11A & $118.8(16)$ \\
\hline $\mathrm{C} 13$ & $\mathrm{C} 12$ & $\mathrm{C} 14$ & $121.0(13)$ & C13A C12AC14A & $126(2)$ \\
\hline $\mathrm{C} 8$ & $\mathrm{C} 13$ & N1 & $121.3(8)$ & C8A C13AN1A & $119.7(13)$ \\
\hline $\mathrm{C} 8$ & $\mathrm{C} 13$ & $\mathrm{C} 12$ & $121.4(8)$ & C12A C13AN1A & $120.1(15)$ \\
\hline $\mathrm{C} 12$ & C13 & N1 & $117.3(9)$ & C12A C13AC8A & $120.0(14)$ \\
\hline $\mathrm{C} 15$ & $\mathrm{C} 14$ & $\mathrm{C} 12$ & $107.8(16)$ & C12A C14AC15A & $117(2)$ \\
\hline $\mathrm{C} 15$ & $\mathrm{C} 14$ & $\mathrm{C} 16$ & $109(2)$ & C12A C14AC16A & $113(3)$ \\
\hline $\mathrm{C} 16$ & C14 & $\mathrm{C} 12$ & $110(2)$ & C15A C14AC16A & $110(3)$ \\
\hline N1 & C18 & $\mathrm{C} 17$ & $123.4(7)$ & N1A C18AC17A & $124.0(15)$ \\
\hline N1 & $\mathrm{C} 18$ & C19 & $114.9(7)$ & N1A C18AC19A & $114.9(11)$ \\
\hline $\mathrm{C} 19$ & $\mathrm{C} 18$ & $\mathrm{C} 17$ & $121.6(7)$ & C19A C18AC17A & $121.1(13)$ \\
\hline $\mathrm{N} 2$ & C19 & $\mathrm{C} 18$ & $110.6(7)$ & N2A C19AC18A & $111.3(12)$ \\
\hline $\mathrm{N} 2$ & C19 & $\mathrm{C} 20$ & $119.6(9)$ & N2A C19AC20A & $119.5(13)$ \\
\hline $\mathrm{C} 20$ & C19 & $\mathrm{C} 18$ & $129.9(8)$ & C18A C19AC20A & $129.2(13)$ \\
\hline C19 & $\mathrm{C} 20$ & $\mathrm{C} 21$ & $118.7(8)$ & C19A C20AC21A & $116.3(12)$ \\
\hline $\mathrm{C} 20$ & $\mathrm{C} 21$ & $\mathrm{C} 22$ & $121.6(8)$ & C22A C21AC20A & $123.3(13)$ \\
\hline $\mathrm{C} 23$ & $\mathrm{C} 22$ & $\mathrm{C} 21$ & $118.4(8)$ & C21A C22AC23A & $118.8(13)$ \\
\hline $\mathrm{N} 2$ & $\mathrm{C} 23$ & $\mathrm{C} 22$ & $119.5(8)$ & $\mathrm{N} 2 \mathrm{~A}$ C23AC22A & $117.4(14)$ \\
\hline $\mathrm{N} 2$ & $\mathrm{C} 23$ & $\mathrm{C} 24$ & $110.1(7)$ & $\mathrm{N} 2 \mathrm{~A}$ C23AC24A & $112.4(12)$ \\
\hline $\mathrm{C} 22$ & $\mathrm{C} 23$ & $\mathrm{C} 24$ & $130.4(9)$ & C22A C23AC24A & $130.1(12)$ \\
\hline N3 & $\mathrm{C} 24$ & $\mathrm{C} 23$ & $114.0(7)$ & N3A C24AC23A & $112.7(11)$ \\
\hline $\mathrm{N} 3$ & $\mathrm{C} 24$ & $\mathrm{C} 25$ & $125.7(8)$ & $\mathrm{N} 3 \mathrm{~A}$ C24AC25A & $126.6(12)$ \\
\hline $\mathrm{C} 23$ & $\mathrm{C} 24$ & $\mathrm{C} 25$ & $120.3(8)$ & C23A C24AC25A & $120.6(11)$ \\
\hline $\mathrm{C} 26$ & $\mathrm{C} 28$ & $\mathrm{C} 27$ & $108.5(14)$ & C26A C28AC27A & $111(2)$ \\
\hline $\mathrm{C} 26$ & C28 & C29 & $115.5(15)$ & C29A C28A C26A & $109(2)$ \\
\hline
\end{tabular}




\begin{tabular}{|c|c|c|c|c|c|}
\hline $\mathrm{C} 29$ & $\mathrm{C} 28$ & $\mathrm{C} 27$ & $107.9(17)$ & C29A C28AC27A & $113(3)$ \\
\hline $\mathrm{C} 30$ & C29 & $\mathrm{C} 28$ & $117.8(11)$ & C30A C29A C28A & $122.5(19)$ \\
\hline $\mathrm{C} 30$ & C29 & $\mathrm{C} 34$ & $119.6(12)$ & C30A C29A C34A & $116.1(18)$ \\
\hline C34 & C29 & $\mathrm{C} 28$ & $122.6(11)$ & C34A C29A C28A & $121.1(18)$ \\
\hline $\mathrm{C} 29$ & $\mathrm{C} 30$ & $\mathrm{C} 31$ & $120.6(11)$ & C31A C30A C29A & $123.6(18)$ \\
\hline $\mathrm{C} 32$ & C31 & $\mathrm{C} 30$ & $118.9(9)$ & C30A C31AC32A & $119.2(16)$ \\
\hline C31 & C32 & $\mathrm{C} 33$ & $121.8(11)$ & C33A C32AC31A & $122.8(17)$ \\
\hline C32 & C33 & $\mathrm{C} 34$ & $118.7(10)$ & C32A C33AC34A & $117.0(16)$ \\
\hline $\mathrm{C} 35$ & C33 & $\mathrm{C} 32$ & $108.4(10)$ & C32A C33AC35A & $133.5(15)$ \\
\hline C35 & C33 & $\mathrm{C} 34$ & $132.8(9)$ & C34A C33AC35A & $108.8(13)$ \\
\hline $\mathrm{C} 29$ & C34 & N3 & $122.7(11)$ & C29A C34AN3A & $120.3(17)$ \\
\hline $\mathrm{C} 29$ & C34 & $\mathrm{C} 33$ & $120.2(9)$ & C29A C34A C33A & $121.1(14)$ \\
\hline C33 & C34 & N3 & $117.0(9)$ & C33A C34AN3A & $118.6(15)$ \\
\hline C33 & C35 & $\mathrm{C} 36$ & $122.3(10)$ & C36A C35AC33A & $107.4(14)$ \\
\hline C33 & $\mathrm{C} 35$ & $\mathrm{C} 37$ & $110.1(11)$ & C36A C35AC37A & $109.5(14)$ \\
\hline C37 & $\mathrm{C} 35$ & $\mathrm{C} 36$ & $107.5(9)$ & C37A C35AC33A & $99.6(13)$ \\
\hline
\end{tabular}

Table S-7. Torsion Angles for 1-PMe

\begin{tabular}{|c|c|c|c|c|c|c|c|}
\hline $\mathbf{A}$ & B & $\mathbf{C}$ & D & Angle ${ }^{\circ}$ & $\mathbf{A}$ & $\mathbf{C}$ & Angle ${ }^{\circ}$ \\
\hline $\mathrm{P} 1$ & $\mathrm{Fe} 1$ & $\mathrm{~N} 2$ & C19 & $-86.9(6)$ & P1A Fe1A & $\mathrm{N} 2 \mathrm{~A} \quad \mathrm{C} 23 \mathrm{~A}$ & $88.0(10)$ \\
\hline $\mathrm{P} 1$ & $\mathrm{Fe} 1$ & $\mathrm{~N} 2$ & $\mathrm{C} 23$ & $87.6(6)$ & Fe1A N1A & C13AC8A & $80.9(19)$ \\
\hline $\mathrm{Fe} 1$ & N1 & C13 & $\mathrm{C} 8$ & $85.1(12)$ & Fe1A N1A & C13AC12A & $-93.8(19)$ \\
\hline e1 & N1 & C13 & $\mathrm{C} 12$ & $-95.9(11)$ & Fe1A N1A & C18AC17A & $171.9(11)$ \\
\hline 1 & $\mathrm{~N} 1$ & C18 & C17 & $-169.8(7)$ & Fe1A N1A & C18A C19A & $8.6(16)$ \\
\hline el & $\mathrm{N} 1$ & C18 & C19 & $7.7(11)$ & Fe1A N2A & C19A C18A & $-12.8(15)$ \\
\hline e1 & $\mathrm{N} 2$ & C19 & C18 & $-11.0(9)$ & Fe1A N2A & C19A C20A & $167.9(10)$ \\
\hline e1 & $\mathrm{N} 2$ & C19 & C20 & $167.5(6)$ & Fe1A N2A & $\mathrm{C} 23 \mathrm{AC} 22 \mathrm{~A}$ & $168.4(10)$ \\
\hline e1 & $\mathrm{N} 2$ & $\mathrm{C} 23$ & $\mathrm{C} 22$ & $-168.8(7)$ & Fe1A N2A & $\mathrm{C} 23 \mathrm{AC} 24 \mathrm{~A}$ & $9.3(15)$ \\
\hline e1 & $\mathrm{N} 2$ & $\mathrm{C} 23$ & $\mathrm{C} 24$ & $11.0(9)$ & Fe1A N3A & $\mathrm{C} 24 \mathrm{AC} 23 \mathrm{~A}$ & $-7.6(17)$ \\
\hline Fe1 & $\mathrm{N} 3$ & C24 & $\mathrm{C} 23$ & $-8.3(11)$ & Fe1A N3A & $\mathrm{C} 24 \mathrm{AC} 25 \mathrm{~A}$ & $176.5(12)$ \\
\hline Fe1 & N3 & C24 & $\mathrm{C} 25$ & $171.7(8)$ & Fe1A N3A & C34A C29A & $-82(2)$ \\
\hline $\mathrm{Fe} 1$ & N3 & C34 & C29 & $-82.1(15)$ & Fe1A N3A & C34A C33A & $99(2)$ \\
\hline $\mathrm{Fe} 1$ & $\mathrm{~N} 3$ & C34 & C33 & $94.3(14)$ & C1A Fe1A & N2A C19A & $98(3)$ \\
\hline N1 & Fe1 & $\mathrm{N} 2$ & C19 & $12.0(7)$ & C1A Fe1A & $\mathrm{N} 2 \mathrm{~A} \quad \mathrm{C} 23 \mathrm{~A}$ & $-88(3)$ \\
\hline N1 & $\mathrm{Fe} 1$ & $\mathrm{~N} 2$ & $\mathrm{C} 23$ & $-173.4(7)$ & N1A Fe1A & N2A C19A & $13.9(11)$ \\
\hline N1 & $\mathrm{C} 18$ & C19 & $\mathrm{N} 2$ & 1. & N1A $F$ & $\mathrm{~N} 2 \mathrm{~A} \mathrm{C} 23 \mathrm{~A}$ & $172.2(12)$ \\
\hline N1 & C18 & C19 & C20 & $-176.7(9)$ & N1A C18A & C19AN2A & $2.0(17)$ \\
\hline
\end{tabular}




\begin{tabular}{|c|c|c|c|c|c|c|}
\hline $\mathrm{N} 2$ & $\mathrm{C} 19$ & $\mathrm{C} 20$ & $\mathrm{C} 21$ & $3.8(11)$ & N1A C18AC19AC20A & $178.7(13)$ \\
\hline N2 & $\mathrm{C} 23$ & $\mathrm{C} 24$ & $\mathrm{~N} 3$ & $-1.2(10)$ & N2A C19AC20AC21A & $3.4(17)$ \\
\hline N2 & $\mathrm{C} 23$ & $\mathrm{C} 24$ & $\mathrm{C} 25$ & $178.8(7)$ & N2A C23AC24AN3A & $-0.6(17)$ \\
\hline 3 & $\mathrm{Fe} 1$ & $\mathrm{~N} 2$ & $\mathrm{C} 19$ & $173.2(7)$ & N2A C 23 AC24AC25A & $175.5(12)$ \\
\hline N3 & $\mathrm{Fe} 1$ & $\mathrm{~N} 2$ & $\mathrm{C} 23$ & $-12.3(7)$ & N3A Fe1A N2A C19A & $175.5(11)$ \\
\hline $\mathrm{C} 5$ & $\mathrm{C} 7$ & $\mathrm{C} 8$ & C9 & $51.7(10)$ & N3A Fe1A N2A C23A & $-10.5(12)$ \\
\hline C5 & $\mathrm{C} 7$ & $\mathrm{C} 8$ & $\mathrm{C} 13$ & $-127.4(9)$ & C5A C7A C8A C9A & $46.8(16)$ \\
\hline C6 & $\mathrm{C} 7$ & $\mathrm{C} 8$ & C9 & $-72.1(10)$ & C5A C7A C8A C13A & $138.5(15)$ \\
\hline C6 & $\mathrm{C} 7$ & $\mathrm{C} 8$ & $\mathrm{C} 13$ & $108.8(10)$ & C6A C7A C8A C9A & $-80.1(16)$ \\
\hline $\mathrm{C} 7$ & $\mathrm{C} 8$ & C9 & $\mathrm{C} 10$ & $-178.5(8)$ & C6A C7A C8A C13A & $94.7(17)$ \\
\hline $\mathrm{C} 7$ & $\mathrm{C} 8$ & $\mathrm{C} 13$ & N1 & $-2.8(13)$ & C7A C8A C9A C10A & $176.2(12)$ \\
\hline $\mathrm{C} 7$ & $\mathrm{C} 8$ & $\mathrm{C} 13$ & $\mathrm{C} 12$ & $178.2(9)$ & C7A C8A C13AN1A & $6(2)$ \\
\hline $\mathrm{C} 8$ & C9 & $\mathrm{C} 10$ & $\mathrm{C} 11$ & 0.3 & C7A C8A C13AC12A & $178.8(14)$ \\
\hline C9 & $\mathrm{C} 8$ & $\mathrm{C} 13$ & $\mathrm{~N} 1$ & $178.0(8)$ & C8A C9A C10AC11A & $2(2)$ \\
\hline C9 & $\mathrm{C} 8$ & $\mathrm{C} 13$ & $\mathrm{C} 12$ & $-1.0(14)$ & C9A C8A C13AN1A & $178.9(13)$ \\
\hline C9 & $\mathrm{C} 10$ & $\mathrm{C} 11$ & $\mathrm{C} 12$ & $-1.0(15)$ & C9A C8A C13AC12A & $-4(2)$ \\
\hline $\mathrm{C} 10$ & $\mathrm{C} 11$ & $\mathrm{C} 12$ & $\mathrm{C} 13$ & 0.7 & C9A C10AC11AC12A & $-2(2)$ \\
\hline $\mathrm{C} 1$ & $\mathrm{C} 11$ & $\mathrm{C} 12$ & $\mathrm{C} 14$ & 78.8 & C10AC11AC12AC13A & $-1(3)$ \\
\hline $\mathrm{C} 1$ & $\mathrm{C} 12$ & $\mathrm{C} 13$ & N1 & 178.7( & 10AC11AC12AC14A & 9) \\
\hline $\mathrm{C} 11$ & $\mathrm{C} 12$ & $\mathrm{C} 13$ & $\mathrm{C} 8$ & $0.3(16)$ & C11AC12AC13AN1A & $178.7(16)$ \\
\hline $\mathrm{C} 11$ & $\mathrm{C} 12$ & $\mathrm{C} 14$ & $\mathrm{C} 15$ & $-52(2)$ & C11AC12AC13AC8A & $4(3)$ \\
\hline $\mathrm{C} 11$ & $\mathrm{C} 12$ & $\mathrm{C} 14$ & $\mathrm{C} 16$ & $68(2)$ & 11A C12A C14A C15A & $-49(4)$ \\
\hline $\mathrm{C} 13$ & N1 & $\mathrm{C} 18$ & $\mathrm{C} 17$ & $16.6(14)$ & C11AC12AC14AC16A & $81(3)$ \\
\hline $\mathrm{C} 13$ & N1 & $\mathrm{C} 18$ & $\mathrm{C} 19$ & $-166.0(8)$ & C13AN1A C18AC17A & $13(2)$ \\
\hline & $\mathrm{C} 8$ & C9 & $\mathrm{C} 10$ & & C13AN1A C18AC19A & $166.1(13)$ \\
\hline $\mathrm{C} 13$ & $\mathrm{C} 12$ & $\mathrm{C} 14$ & $\mathrm{C} 15$ & $126.3(18)$ & C13AC8A C9A C10A & $1(2)$ \\
\hline $\mathrm{C} 13$ & $\mathrm{C} 12$ & $\mathrm{C} 14$ & $\mathrm{C} 16$ & $-114(2)$ & C13AC12AC14AC15A & $129(3)$ \\
\hline $\mathrm{C} 14$ & $\mathrm{C} 12$ & $\mathrm{C} 13$ & N1 & $3.2(16)$ & C13AC12AC14AC16A & $-101(4)$ \\
\hline $\mathrm{C} 14$ & $\mathrm{C} 12$ & $\mathrm{C} 13$ & $\mathrm{C} 8$ & $177.8(12)$ & C14AC12AC13AN1A & $1(3)$ \\
\hline $\mathrm{C} 17$ & $\mathrm{C} 18$ & C19 & $\mathrm{N} 2$ & $179.1(7)$ & C14AC12AC13AC8A & $-174(2)$ \\
\hline $\mathrm{C} 17$ & $\mathrm{C} 18$ & C19 & $\mathrm{C} 20$ & $0.7(13$ & C17AC18AC19AN2A & $177.5(11)$ \\
\hline C18 & N1 & $\mathrm{C} 13$ & $\mathrm{C} 8$ & 102 & C17AC18AC19AC20A & $2(2)$ \\
\hline $\mathrm{C} 18$ & N1 & $\mathrm{C} 13$ & $\mathrm{C} 12$ & 12 & C18AN1A C13AC8A & $5.4(18)$ \\
\hline
\end{tabular}




\begin{tabular}{|c|c|c|c|c|}
\hline C18 C19 & $\mathrm{C} 20 \mathrm{C} 21$ & $-177.9(8)$ & C18AN1A C13AC12A & $80(2)$ \\
\hline C19 N2 & $\mathrm{C} 23 \mathrm{C} 22$ & $5.5(11)$ & C18AC19AC20A C21A & $175.8(12)$ \\
\hline C19 N2 & $\mathrm{C} 23 \mathrm{C} 24$ & $-174.6(7)$ & C19AN2A C23AC22A & $5.1(19)$ \\
\hline $\mathrm{C} 19 \mathrm{C} 20$ & $\mathrm{C} 21 \mathrm{C} 22$ & $0.2(12)$ & C19AN2A C23AC24A & $177.2(12)$ \\
\hline C20 C21 & $\mathrm{C} 22 \mathrm{C} 23$ & $-1.5(12)$ & C19A C20AC21AC22A & $-1.1(18)$ \\
\hline $\mathrm{C} 21 \mathrm{C} 22$ & $\mathrm{C} 23 \mathrm{~N} 2$ & $-1.3(12)$ & C20AC21AC22A C23A & $0.7(19)$ \\
\hline $\mathrm{C} 21 \mathrm{C} 22$ & $\mathrm{C} 23 \mathrm{C} 24$ & $178.9(8)$ & C21AC22AC23AN2A & $-2.5(18)$ \\
\hline $\mathrm{C} 22 \mathrm{C} 23$ & $\mathrm{C} 24 \mathrm{~N} 3$ & $178.6(9)$ & C21A C22AC23A C24A & 179 \\
\hline $\mathrm{C} 22 \mathrm{C} 23$ & $\mathrm{C} 24 \mathrm{C} 25$ & $-1.4(13)$ & C22A C23A C24AN3A & $176.7(15)$ \\
\hline $\mathrm{C} 23 \mathrm{~N} 2$ & C19 C18 & $174.6(7)$ & C22A C23A C24A C25A & $-7(2)$ \\
\hline C23 N2 & C19 C20 & $-6.8(11)$ & 23AN2A C19AC18A & $173.7(12)$ \\
\hline C24 N3 & C34 C29 & $105.9(14)$ & C23AN2A C19AC20A & $-5.6(19)$ \\
\hline C24 N3 & C34 C33 & $-77.8(15)$ & C24AN3A C34AC29A & $109(2)$ \\
\hline C26 C28 & C29 C30 & $-41(2)$ & C24AN3A C34AC33A & $-69(2)$ \\
\hline C26 C28 & C29 C34 & $139.6(18)$ & 26A C28A C29A C30A & $-55(4)$ \\
\hline $\mathrm{C} 27 \mathrm{C} 28$ & C29 C30 & $80.9(17)$ & $26 \mathrm{AC} 28 \mathrm{AC} 29 \mathrm{AC} 34 \mathrm{~A}$ & $131(3)$ \\
\hline $\mathrm{C} 27 \mathrm{C} 28$ & C29 C34 & $-98.9(17)$ & 27A C28A C29A C30A & $70(3)$ \\
\hline C28 C29 & C30 C31 & $177.8(16)$ & C27A C28A C29A C34A & (3) \\
\hline C28 C29 & C34 N3 & $-3(2)$ & C28AC29A C30A C31A & $-178(3)$ \\
\hline C28 C29 & C34 C33 & $178.8(15)$ & C28AC29A C34AN3A & $-3(3)$ \\
\hline C29 C30 & C31 C32 & $-3(2)$ & C28A C29A C34A C33A & $176(2)$ \\
\hline C30 C29 & C34 N3 & $177.6(12)$ & $29 \mathrm{AC} 30 \mathrm{AC} 31 \mathrm{AC} 32 \mathrm{~A}$ & $2(4)$ \\
\hline C30 C29 & C34 C33 & $1(2)$ & C30A C29A C34AN3A & $176.5(19)$ \\
\hline C30 C31 & C32 C33 & $0(2)$ & C30A C29A C34A C33A & $2(3)$ \\
\hline C31 C32 & C33 C34 & $3.4(19)$ & C30AC31AC32AC33A & $2(3)$ \\
\hline C31 C32 & C33 C35 & $178.5(13)$ & C31A C32A C33A C34A & $-4(3)$ \\
\hline C32 C33 & C34 N3 & $179.5(11)$ & C31AC32AC33AC35A & $-173(2)$ \\
\hline C32 C33 & $\mathrm{C} 34 \mathrm{C} 29$ & $-4.0(19)$ & C32AC33AC34AN3A & $179.7(17)$ \\
\hline C32 C33 & C35 C36 & $-33.0(17)$ & C32A C33A C34A C29A & $1(3)$ \\
\hline C32 C33 & C35 C37 & $94.6(12)$ & C32A C33AC35A C36A & $-81(2)$ \\
\hline C34 N3 & C24 C23 & $164.6(9)$ & C32A C33A C35A C37A & $33(3)$ \\
\hline C34 N3 & $\mathrm{C} 24 \mathrm{C} 25$ & $-15.4(15)$ & C34AN3A C24AC23A & $162.7(13)$ \\
\hline C34 C29 & C30 C31 & $2(2)$ & C34AN3A C24AC25A & $-13(2)$ \\
\hline C34 C33 & C35 C36 & $144.7(16)$ & C34A C29A C30A C31A & $-4(3)$ \\
\hline C34 C33 & C35 C37 & $-87.7(19)$ & C34A C33A C35A C36A & $109.0(18)$ \\
\hline
\end{tabular}




$\begin{array}{llrlr}\text { C35 C33 C34 N3 } & 2(2) & \text { C34AC33AC35AC37A } & 136.9(17) \\ \text { C35 C33 C34 C29 } & 178.4(15) & \text { C35AC33AC34AN3A } & -8(2) \\ \text { P1AFe1A N2AC19A } & -86.0(10) & \text { C35AC33AC34AC29A } & 173.6(18)\end{array}$

Table S-8. Hydrogen Atom Coordinates $\left(\AA \times 10^{4}\right)$ and Isotropic Displacement Parameters $\left(\AA^{2} \times 10^{3}\right)$ for $\mathbf{1}-\mathbf{P M e}_{3}$.

\begin{tabular}{|c|c|c|c|c|}
\hline Atom & $x$ & $y$ & $z$ & $\mathbf{U}(\mathbf{e q})$ \\
\hline $\mathrm{H} 2 \mathrm{~A}$ & 9356 & 8777 & 5245 & 44 \\
\hline $\mathrm{H} 2 \mathrm{~B}$ & 9408 & 8160 & 6108 & 44 \\
\hline $\mathrm{H} 2 \mathrm{C}$ & 8731 & 9026 & 5833 & 44 \\
\hline H3A & 7586 & 8626 & 4235 & 47 \\
\hline H3B & 7708 & 7562 & 3740 & 47 \\
\hline $\mathrm{H} 3 \mathrm{C}$ & 8392 & 8437 & 3900 & 47 \\
\hline $\mathrm{H} 4 \mathrm{~A}$ & 9452 & 7019 & 4453 & 60 \\
\hline H4B & 8811 & 6085 & 4321 & 60 \\
\hline $\mathrm{H} 4 \mathrm{C}$ & 9411 & 6195 & 5187 & 60 \\
\hline H1A & 8092 & 7007 & 7288 & 47 \\
\hline H1B & 7193 & 7402 & 7123 & 47 \\
\hline $\mathrm{H} 1 \mathrm{C}$ & 7858 & 8102 & 6811 & 47 \\
\hline H5A & 8061 & 10454 & 6129 & 70 \\
\hline H5B & 7729 & 9712 & 6781 & 70 \\
\hline H5C & 7295 & 10807 & 6487 & 70 \\
\hline H6A & 6542 & 10963 & 4988 & 67 \\
\hline H6B & 6519 & 9965 & 4381 & 67 \\
\hline H6C & 7328 & 10600 & 4672 & 67 \\
\hline $\mathrm{H} 7$ & 7356 & 9010 & 5424 & 29 \\
\hline H9 & 5953 & 10550 & 6317 & 34 \\
\hline $\mathrm{H} 10$ & 4840 & 9880 & 6732 & 37 \\
\hline H11 & 4507 & 8121 & 6543 & 35 \\
\hline H14 & 5625 & 6120 & 5651 & 39 \\
\hline H15A & 5589 & 6163 & 7098 & 65 \\
\hline H15B & 4967 & 5311 & 6643 & 65 \\
\hline $\mathrm{H} 15 \mathrm{C}$ & 4667 & 6446 & 6894 & 65 \\
\hline H16A & 3992 & 6779 & 5431 & 70 \\
\hline H16B & 4267 & 5628 & 5186 & 70 \\
\hline $\mathrm{H} 16 \mathrm{C}$ & 4476 & 6651 & 4690 & 70 \\
\hline H17A & 5330 & 8194 & 4283 & 34 \\
\hline H17B & 5277 & 7296 & 3584 & 34 \\
\hline $\mathrm{H} 17 \mathrm{C}$ & 5880 & 8265 & 3595 & 34 \\
\hline
\end{tabular}




\begin{tabular}{|c|c|c|c|c|}
\hline H20 & 6163 & 6150 & 2959 & 31 \\
\hline $\mathrm{H} 21$ & 6883 & 4634 & 2729 & 32 \\
\hline $\mathrm{H} 22$ & 7769 & 3837 & 3820 & 36 \\
\hline $\mathrm{H} 25 \mathrm{~A}$ & 9026 & 3754 & 5229 & 71 \\
\hline $\mathrm{H} 25 \mathrm{~B}$ & 8248 & 3053 & 5216 & 71 \\
\hline $\mathrm{H} 25 \mathrm{C}$ & 8759 & 3435 & 6083 & 71 \\
\hline $\mathrm{H} 26 \mathrm{~A}$ & 10346 & 6537 & 8125 & 63 \\
\hline H26B & 9615 & 7315 & 7822 & 63 \\
\hline $\mathrm{H} 26 \mathrm{C}$ & 10359 & 7325 & 7361 & 63 \\
\hline $\mathrm{H} 27 \mathrm{~A}$ & 10638 & 5843 & 6515 & 89 \\
\hline H27B & 10040 & 4866 & 6315 & 89 \\
\hline $\mathrm{H} 27 \mathrm{C}$ & 10583 & 4977 & 7216 & 89 \\
\hline $\mathrm{H} 28$ & 9311 & 6387 & 6545 & 44 \\
\hline H30 & 9811 & 5151 & 8512 & 40 \\
\hline H31 & 9047 & 3893 & 9089 & 42 \\
\hline H32 & 7847 & 3341 & 8323 & 41 \\
\hline H35 & 7110 & 3762 & 6391 & 55 \\
\hline H36A & 7388 & 2164 & 7223 & 130 \\
\hline H36B & 6475 & 2402 & 6870 & 130 \\
\hline H36C & 6840 & 2651 & 7821 & 130 \\
\hline H37A & 6497 & 4523 & 7772 & 94 \\
\hline H37B & 6000 & 4275 & 6867 & 94 \\
\hline H37C & 6614 & 5239 & 6998 & 94 \\
\hline H3AA & 7833 & 8116 & 4094 & 51 \\
\hline $\mathrm{H} 3 \mathrm{AB}$ & 8032 & 6987 & 3734 & 51 \\
\hline $\mathrm{H} 3 \mathrm{AC}$ & 8689 & 7901 & 3876 & 51 \\
\hline $\mathrm{H} 2 \mathrm{AA}$ & 9568 & 8423 & 5210 & 43 \\
\hline $\mathrm{H} 2 \mathrm{AB}$ & 9582 & 7937 & 6116 & 43 \\
\hline $\mathrm{H} 2 \mathrm{AC}$ & 8917 & 8780 & 5739 & 43 \\
\hline H4AA & 9022 & 5543 & 4620 & 47 \\
\hline $\mathrm{H} 4 \mathrm{AB}$ & 9603 & 5863 & 5463 & 47 \\
\hline $\mathrm{H} 4 \mathrm{AC}$ & 9664 & 6460 & 4615 & 47 \\
\hline H1AA & 8186 & 7307 & 7196 & 47 \\
\hline $\mathrm{H} 1 \mathrm{AB}$ & 7384 & 6719 & 7296 & 47 \\
\hline $\mathrm{H} 1 \mathrm{AC}$ & 7350 & 7845 & 6840 & 47 \\
\hline H5AA & 8271 & 10248 & 5677 & 79 \\
\hline $\mathrm{H} 5 \mathrm{AB}$ & 7830 & 9931 & 6421 & 79 \\
\hline H5AC & 7504 & 10892 & 5815 & 79 \\
\hline H6AA & 6630 & 10443 & 4375 & 83 \\
\hline H6AB & 6766 & 9298 & 4005 & 83 \\
\hline H6AC & 7484 & 10120 & 4201 & 83 \\
\hline H7A & 7551 & 8764 & 5232 & 46 \\
\hline
\end{tabular}




\begin{tabular}{|c|c|c|c|c|}
\hline H9A & 6207 & 10549 & 5921 & 36 \\
\hline H10A & 5001 & 10098 & 6335 & 33 \\
\hline H11A & 4639 & 8333 & 6285 & 31 \\
\hline H14A & 5664 & 6166 & 5762 & 34 \\
\hline H15D & 5454 & 6478 & 7129 & 46 \\
\hline $\mathrm{H} 15 \mathrm{E}$ & 4923 & 5535 & 6669 & 46 \\
\hline $\mathrm{H} 15 \mathrm{~F}$ & 4537 & 6673 & 6764 & 46 \\
\hline H16D & 4108 & 6894 & 5132 & 59 \\
\hline H16E & 4350 & 5668 & 5198 & 59 \\
\hline $\mathrm{H} 16 \mathrm{~F}$ & 4727 & 6439 & 4600 & 59 \\
\hline H17D & 5545 & 7838 & 4074 & 45 \\
\hline H17E & 5493 & 6793 & 3511 & 45 \\
\hline $\mathrm{H} 17 \mathrm{~F}$ & 6105 & 7713 & 3400 & 45 \\
\hline $\mathrm{H} 20 \mathrm{~A}$ & 6407 & 5548 & 3021 & 28 \\
\hline $\mathrm{H} 21 \mathrm{~A}$ & 7099 & 3930 & 3045 & 35 \\
\hline $\mathrm{H} 22 \mathrm{~A}$ & 7939 & 3327 & 4231 & 39 \\
\hline $\mathrm{H} 25 \mathrm{D}$ & 9133 & 3481 & 6371 & 45 \\
\hline $\mathrm{H} 25 \mathrm{E}$ & 8785 & 3078 & 5449 & 45 \\
\hline $\mathrm{H} 25 \mathrm{~F}$ & 8310 & 2856 & 6186 & 45 \\
\hline$H 26 D$ & 10251 & 6790 & 8272 & 63 \\
\hline $\mathrm{H} 26 \mathrm{E}$ & 9433 & 7340 & 7867 & 63 \\
\hline $\mathrm{H} 26 \mathrm{~F}$ & 10197 & 7492 & 7449 & 63 \\
\hline H27D & 10711 & 5908 & 6724 & 89 \\
\hline $\mathrm{H} 27 \mathrm{E}$ & 10246 & 4804 & 6681 & 89 \\
\hline $\mathrm{H} 27 \mathrm{~F}$ & 10749 & 5208 & 7546 & 89 \\
\hline $\mathrm{H} 28 \mathrm{~A}$ & 9361 & 6247 & 6673 & 44 \\
\hline H30A & 9898 & 5175 & 8747 & 40 \\
\hline H31A & 9136 & 4099 & 9382 & 42 \\
\hline H32A & 7930 & 3490 & 8654 & 41 \\
\hline H35A & 6928 & 4460 & 6372 & 55 \\
\hline H36D & 7376 & 2770 & 6067 & 74 \\
\hline H36E & 6530 & 2541 & 6304 & 74 \\
\hline $\mathrm{H} 36 \mathrm{~F}$ & 7319 & 2332 & 6972 & 74 \\
\hline H37D & 6539 & 3376 & 7810 & 69 \\
\hline H37E & 5919 & 3994 & 7126 & 69 \\
\hline $\mathrm{H} 37 \mathrm{~F}$ & 6561 & 4643 & 7766 & \\
\hline
\end{tabular}

Table S-9. Atomic Occupancy for 1-PMe 3 .
Atom Occupancy
Atom Occupancy
Atom Occupancy
P1
$0.603(6)$
C2
0.603 (6) H2A
0.603 (6) 


\begin{tabular}{|c|c|c|c|c|c|}
\hline $\mathrm{H} 2 \mathrm{~B}$ & $0.603(6)$ & $\mathrm{H} 2 \mathrm{C}$ & $0.603(6)$ & $\mathrm{C} 3$ & $0.603(6)$ \\
\hline $\mathrm{H} 3 \mathrm{~A}$ & $0.603(6)$ & H3B & $0.603(6)$ & $\mathrm{H} 3 \mathrm{C}$ & $0.603(6)$ \\
\hline $\mathrm{C} 4$ & $0.603(6)$ & $\mathrm{H} 4 \mathrm{~A}$ & $0.603(6)$ & $\mathrm{H} 4 \mathrm{~B}$ & $0.603(6)$ \\
\hline $\mathrm{H} 4 \mathrm{C}$ & $0.603(6)$ & $\mathrm{Fe} 1$ & $0.603(6)$ & $\mathrm{C} 1$ & $0.397(6$ \\
\hline $\mathrm{H} 1 \mathrm{~A}$ & $0.397(6)$ & H1B & $0.397(6)$ & $\mathrm{H} 1 \mathrm{C}$ & $0.397(6$ \\
\hline N1 & $0.603(6)$ & $\mathrm{N} 2$ & $0.603(6)$ & N3 & $0.603(6$ \\
\hline C5 & $0.603(6)$ & $\mathrm{H} 5 \mathrm{~A}$ & $0.603(6)$ & H5B & 0.60316 \\
\hline $\mathrm{H} 5 \mathrm{C}$ & $0.603(6)$ & C6 & $0.603(6)$ & H6A & 0.60316 \\
\hline H6B & $0.603(6)$ & H6C & $0.603(6)$ & $\mathrm{C} 7$ & 0.60316 \\
\hline $\mathrm{H} 7$ & $0.603(6)$ & $\mathrm{C} 8$ & $0.603(6)$ & C9 & 0.60316 \\
\hline H9 & $0.603(6)$ & $\mathrm{C} 10$ & $0.603(6)$ & H10 & 0.60316 \\
\hline C11 & $0.603(6)$ & H11 & $0.603(6)$ & $\mathrm{C} 12$ & 0.60316 \\
\hline C13 & $0.603(6)$ & C14 & $0.603(6)$ & H14 & 0.60316 \\
\hline $\mathrm{C} 15$ & $0.603(6)$ & H15A & $0.603(6)$ & H15B & 0.60316 \\
\hline $\mathrm{H} 15 \mathrm{C}$ & $0.603(6)$ & $\mathrm{C} 16$ & $0.603(6)$ & H16A & $0.603(6$ \\
\hline H16B & $0.603(6)$ & H16C & $0.603(6)$ & $\mathrm{C} 17$ & $0.603(6$ \\
\hline H17A & $0.603(6)$ & H17B & $0.603(6)$ & $\mathrm{H} 17 \mathrm{C}$ & $0.603(6$ \\
\hline $\mathrm{C} 18$ & $0.603(6)$ & C19 & $0.603(6)$ & $\mathrm{C} 20$ & 0.60316 \\
\hline $\mathrm{H} 20$ & $0.603(6)$ & $\mathrm{C} 21$ & $0.603(6)$ & $\mathrm{H} 21$ & 0.60316 \\
\hline $\mathrm{C} 22$ & $0.603(6)$ & $\mathrm{H} 22$ & $0.603(6)$ & $\mathrm{C} 23$ & $0.603(6$ \\
\hline $\mathrm{C} 24$ & $0.603(6)$ & $\mathrm{C} 25$ & $0.603(6)$ & $\mathrm{H} 25 \mathrm{~A}$ & $0.603(6$ \\
\hline $\mathrm{H} 25 \mathrm{~B}$ & $0.603(6)$ & $\mathrm{H} 25 \mathrm{C}$ & $0.603(6)$ & $\mathrm{C} 26$ & $0.603(6$ \\
\hline $\mathrm{H} 26 \mathrm{~A}$ & $0.603(6)$ & H26B & $0.603(6)$ & $\mathrm{H} 26 \mathrm{C}$ & $0.603(6$ \\
\hline $\mathrm{C} 27$ & $0.603(6)$ & H27A & $0.603(6)$ & H27B & 0.60316 \\
\hline $\mathrm{H} 27 \mathrm{C}$ & $0.603(6)$ & $\mathrm{C} 28$ & $0.603(6)$ & $\mathrm{H} 28$ & 0.60316 \\
\hline $\mathrm{C} 29$ & $0.603(6)$ & C30 & $0.603(6)$ & H30 & 0.60316 \\
\hline C31 & $0.603(6)$ & H31 & $0.603(6)$ & C32 & $0.603(6$ \\
\hline H32 & $0.603(6)$ & C33 & $0.603(6)$ & C34 & $0.603(6$ \\
\hline $\mathrm{C} 35$ & $0.603(6)$ & H35 & $0.603(6)$ & C36 & $0.603(6$ \\
\hline H36A & $0.603(6)$ & H36B & $0.603(6)$ & $\mathrm{H} 36 \mathrm{C}$ & 0.60316 \\
\hline C37 & $0.603(6)$ & H37A & $0.603(6)$ & H37B & 0.60316 \\
\hline H37C & $0.603(6)$ & $\mathrm{P} 1 \mathrm{~A}$ & $0.397(6)$ & $\mathrm{C} 3 \mathrm{~A}$ & $0.397(6$ \\
\hline H3AA & $0.397(6)$ & H3AB & $0.397(6)$ & $\mathrm{H} 3 \mathrm{AC}$ & $0.397(6$ \\
\hline $\mathrm{C} 2 \mathrm{~A}$ & $0.397(6)$ & H2AA & $0.397(6)$ & $\mathrm{H} 2 \mathrm{AB}$ & $0.397(6$ \\
\hline $\mathrm{H} 2 \mathrm{AC}$ & $0.397(6)$ & $\mathrm{C} 4 \mathrm{~A}$ & $0.397(6)$ & H4AA & $0.397(6$ \\
\hline $\mathrm{H} 4 \mathrm{AB}$ & $0.397(6)$ & $\mathrm{H} 4 \mathrm{AC}$ & $0.397(6)$ & Fe1A & $0.397(6$ \\
\hline $\mathrm{C} 1 \mathrm{~A}$ & $0.603(6)$ & H1AA & $0.603(6)$ & $\mathrm{H} 1 \mathrm{AB}$ & 0.60316 \\
\hline $\mathrm{H} 1 \mathrm{AC}$ & $0.603(6)$ & $\mathrm{N} 1 \mathrm{~A}$ & $0.397(6)$ & $\mathrm{N} 2 \mathrm{~A}$ & $0.397(6$ \\
\hline N3A & $0.397(6)$ & $\mathrm{C} 5 \mathrm{~A}$ & $0.397(6)$ & H5AA & $0.397(6$ \\
\hline H5AB & $0.397(6)$ & H5AC & $0.397(6)$ & C6A & $0.397(6$ \\
\hline H6AA & $0.397(6)$ & H6AB & $0.397(6)$ & H6AC & $0.397(6$ \\
\hline C7A & $0.397(6)$ & H7A & $0.397(6)$ & C8A & $0.397(6$ \\
\hline
\end{tabular}




$\begin{array}{llllll}\text { C9A } & 0.397(6) & \mathrm{H} 9 \mathrm{~A} & 0.397(6) & \mathrm{C} 10 \mathrm{~A} & 0.397(6) \\ \mathrm{H} 10 \mathrm{~A} & 0.397(6) & \mathrm{C} 11 \mathrm{~A} & 0.397(6) & \mathrm{H} 11 \mathrm{~A} & 0.397(6) \\ \mathrm{C} 12 \mathrm{~A} & 0.397(6) & \mathrm{C} 13 \mathrm{~A} & 0.397(6) & \mathrm{C} 14 \mathrm{~A} & 0.397(6) \\ \mathrm{H} 14 \mathrm{~A} & 0.397(6) & \mathrm{C} 15 \mathrm{~A} & 0.397(6) & \mathrm{H} 15 \mathrm{D} & 0.397(6) \\ \mathrm{H} 15 \mathrm{E} & 0.397(6) & \mathrm{H} 15 \mathrm{~F} & 0.397(6) & \mathrm{C} 16 \mathrm{~A} & 0.397(6) \\ \mathrm{H} 16 \mathrm{D} & 0.397(6) & \mathrm{H} 16 \mathrm{E} & 0.397(6) & \mathrm{H} 16 \mathrm{~F} & 0.397(6) \\ \mathrm{C} 17 \mathrm{~A} & 0.397(6) & \mathrm{H} 17 \mathrm{D} & 0.397(6) & \mathrm{H} 17 \mathrm{E} & 0.397(6) \\ \mathrm{H} 17 \mathrm{~F} & 0.397(6) & \mathrm{C} 18 \mathrm{~A} & 0.397(6) & \mathrm{C} 19 \mathrm{~A} & 0.397(6) \\ \mathrm{C} 20 \mathrm{~A} & 0.397(6) & \mathrm{H} 20 \mathrm{~A} & 0.397(6) & \mathrm{C} 21 \mathrm{~A} & 0.397(6) \\ \mathrm{H} 21 \mathrm{~A} & 0.397(6) & \mathrm{C} 22 \mathrm{~A} & 0.397(6) & \mathrm{H} 22 \mathrm{~A} & 0.397(6) \\ \mathrm{C} 23 \mathrm{~A} & 0.397(6) & \mathrm{C} 24 \mathrm{~A} & 0.397(6) & \mathrm{C} 25 \mathrm{~A} & 0.397(6) \\ \mathrm{H} 25 \mathrm{D} & 0.397(6) & \mathrm{H} 25 \mathrm{E} & 0.397(6) & \mathrm{H} 25 \mathrm{~F} & 0.397(6) \\ \mathrm{C} 26 \mathrm{~A} & 0.397(6) & \mathrm{H} 26 \mathrm{D} & 0.397(6) & \mathrm{H} 26 \mathrm{E} & 0.397(6) \\ \mathrm{H} 26 \mathrm{~F} & 0.397(6) & \mathrm{C} 27 \mathrm{~A} & 0.397(6) & \mathrm{H} 27 \mathrm{D} & 0.397(6) \\ \mathrm{H} 27 \mathrm{E} & 0.397(6) & \mathrm{H} 27 \mathrm{~F} & 0.397(6) & \mathrm{C} 28 \mathrm{~A} & 0.397(6) \\ \mathrm{H} 28 \mathrm{~A} & 0.397(6) & \mathrm{C} 29 \mathrm{~A} & 0.397(6) & \mathrm{C} 30 \mathrm{~A} & 0.397(6) \\ \mathrm{H} 30 \mathrm{~A} & 0.397(6) & \mathrm{C} 31 \mathrm{~A} & 0.397(6) & \mathrm{H} 31 \mathrm{~A} & 0.397(6) \\ \mathrm{C} 32 \mathrm{~A} & 0.397(6) & \mathrm{H} 32 \mathrm{~A} & 0.397(6) & \mathrm{C} 33 \mathrm{~A} & 0.397(6) \\ \mathrm{C} 34 \mathrm{~A} & 0.397(6) & \mathrm{C} 35 \mathrm{~A} & 0.397(6) & \mathrm{H} 35 \mathrm{~A} & 0.397(6) \\ \mathrm{C} 36 \mathrm{~A} & 0.397(6) & \mathrm{H} 36 \mathrm{D} & 0.397(6) & \mathrm{H} 36 \mathrm{E} & 0.397(6) \\ \mathrm{H} 36 \mathrm{~F} & 0.397(6) & \mathrm{C} 37 \mathrm{~A} & 0.397(6) & \mathrm{H} 37 \mathrm{D} & 0.397(6) \\ \mathrm{H} 37 \mathrm{E} & 0.397(6) & \mathrm{H} 37 \mathrm{~F} & 0.397(6) & & \\ & 0.397 & & & \end{array}$

\section{Crystallographic Report for 4}

General information: A brick shaped crystal $(0.12 \times 0.14 \times 0.32 \mathrm{~mm})$ was selected under a stereo-microscope while immersed in Fluorolube oil and mounted using a tapered glass fiber. The diffraction data were measured at $100 \mathrm{~K}$ on a Bruker D8 VENTURE with PHOTON 100 CMOS detector system equipped with a Mo-target X-ray tube $(\lambda=0.71073 \AA)$. Data were collected using $\phi$ and $\omega$ scans to survey a full sphere of reciprocal space with an integration time of $35 \mathrm{sec} /$ frame and a scan width of 1 degree. Data reduction and integration were performed with the Bruker APEX2 software package (Bruker AXS, version 2014.9-0, 2014). Data were corrected for absorption effects using multi-scan procedure as implemented in SADABS (Bruker AXS, version 2014/4, 2014, part of Bruker APEX2 software package). The structure was solved by SHELXT (Sheldrick, G. M. Acta Cryst. 2015, A71, 3-8) and refined by a full-matrix leastsquares procedure using Bruker SHELXTL (version 6.14) software package (XL refinement 
program version 2014/7, Sheldrick, G. M. Acta Cryst. 2008, A64, 112-122; Sheldrick, G. M. Acta Cryst. 2015, C71, 3-8). Crystallographic data and details of the data collection and structure refinement are listed in Table 1.

Specific details for structure refinement: All elements were refined with anisotropic thermal parameters. Hydrogen atoms were included in idealized positions for structure factor calculations. All structures are drawn with thermal ellipsoids at $40 \%$ probability.

Table S-10. Crystal data and structure refinement for 4 .

Identification code

Empirical formula

Formula weight

Temperature

Wavelength

Crystal system

Space group

Unit cell dimensions

Volume

$\mathrm{Z}$

Density (calculated)

Absorption coefficient

$\mathrm{F}(000)$

Crystal size

Theta range for data collection

Index ranges

Reflections collected

Independent reflections

Completeness to theta $=25.076^{\circ}$

Refinement method

Data / restraints / parameters

Goodness-of-fit on $\mathrm{F}^{2}$

Final R indices [I $>2 \operatorname{sigma}(\mathrm{I})]$

$\mathrm{R}$ indices (all data)
4

$\mathrm{C}_{59} \mathrm{H}_{66} \mathrm{BFeN}_{3} \mathrm{O}_{2}$

915.80

100(2) K

$0.71073 \AA$

Monoclinic

$\mathrm{P} 2{ }_{1} / \mathrm{c}$

$\mathrm{a}=12.5740(8) \AA$ $\alpha=90^{\circ}$.

$\mathrm{b}=12.6375(8) \AA$ $\beta=96.473(2)^{\circ}$.

$\mathrm{c}=31.6380(19) \AA \quad \gamma=90^{\circ}$. 4995.4(5) $\AA^{3}$

4

$1.218 \mathrm{Mg} / \mathrm{m}^{3}$

$0.348 \mathrm{~mm}^{-1}$

1952

$0.320 \times 0.140 \times 0.120 \mathrm{~mm}^{3}$

2.068 to $25.076^{\circ}$.

$-14<=\mathrm{h}<=14,-14<=\mathrm{k}<=15,-37<=\mathrm{l}<=36$

102011

8454 [R(int $)=0.0568]$

$95.4 \%$

Full-matrix least-squares on $\mathrm{F}^{2}$

8454 / 0 / 606

1.073

$\mathrm{R} 1=0.0530, \mathrm{wR}_{2}=0.0995$

$\mathrm{R} 1=0.0847, \mathrm{wR} 2=0.1084$ 
Extinction coefficient

Largest diff. peak and hole $\mathrm{n} / \mathrm{a}$

0.402 and -0.312 e. $\AA^{-3}$

$\mathrm{R}_{\mathrm{int}}=\Sigma\left|\mathrm{F}_{\mathrm{o}}^{2}-\left\langle\mathrm{F}_{\mathrm{o}}^{2}\right\rangle\right| / \Sigma\left|\mathrm{F}_{\mathrm{o}}^{2}\right|$

$\mathrm{R} 1=\Sigma|| \mathrm{F}_{\mathrm{o}}|-| \mathrm{F}_{\mathrm{c}} \| / \Sigma\left|\mathrm{F}_{\mathrm{o}}\right|$

$\mathrm{wR} 2=\left[\Sigma\left[\mathrm{w}\left(\mathrm{F}_{\mathrm{o}}^{2}-\mathrm{F}_{\mathrm{c}}^{2}\right)^{2}\right] / \Sigma\left[\mathrm{w}\left(\mathrm{F}_{\mathrm{o}}^{2}\right)^{2}\right]\right]^{1 / 2}$

Goodness-of-fit $=\left[\Sigma\left[\mathrm{w}\left(\mathrm{F}_{\mathrm{o}}{ }^{2-} \mathrm{F}_{\mathrm{c}}{ }^{2}\right)^{2}\right] /(\mathrm{n}-\mathrm{p})^{1 / 2}\right.$

$\mathrm{n}$ : number of independent reflections; $\mathrm{p}$ : number of refined parameters

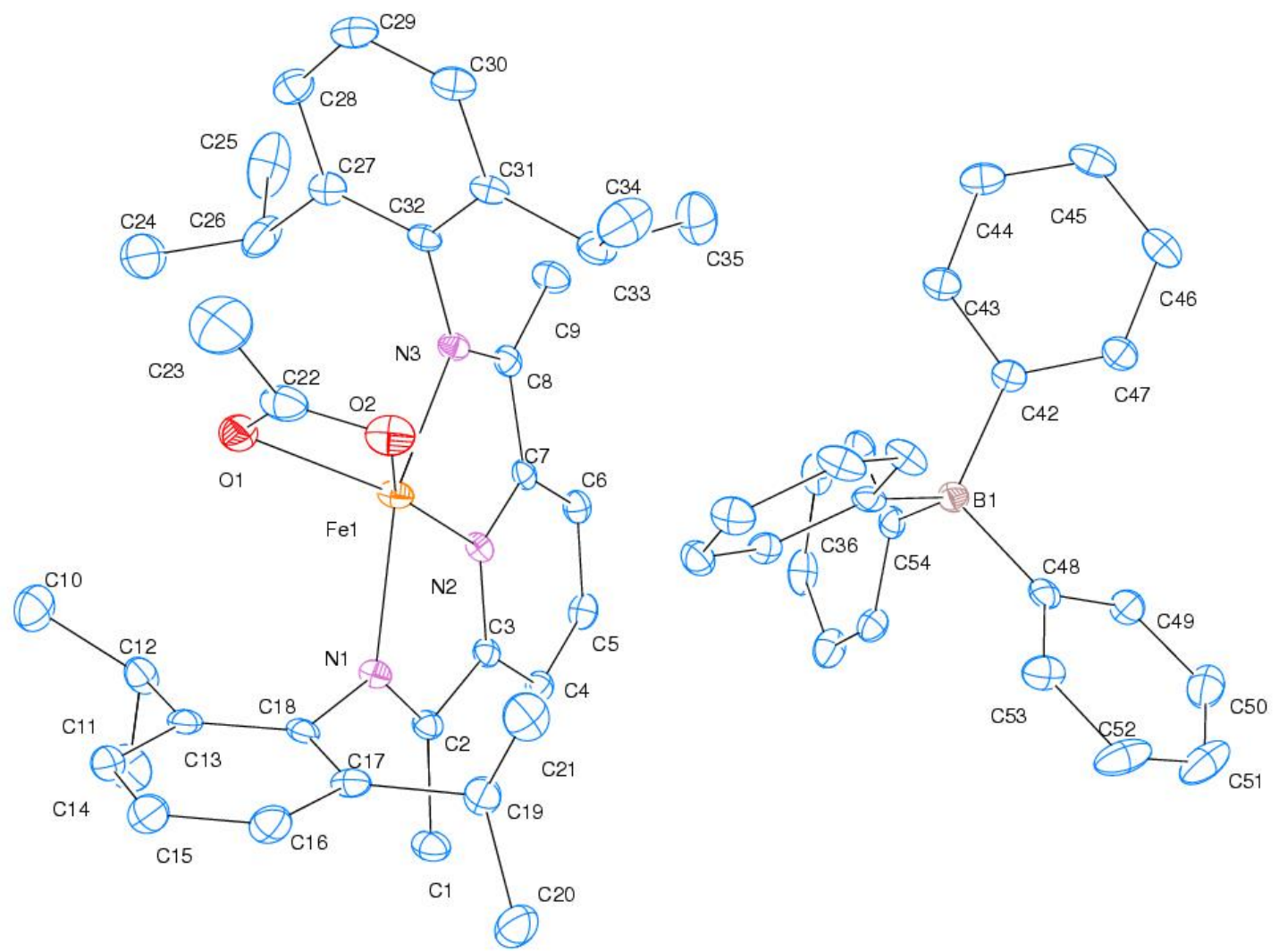

Figure S-26. Molecular structure of 4. 


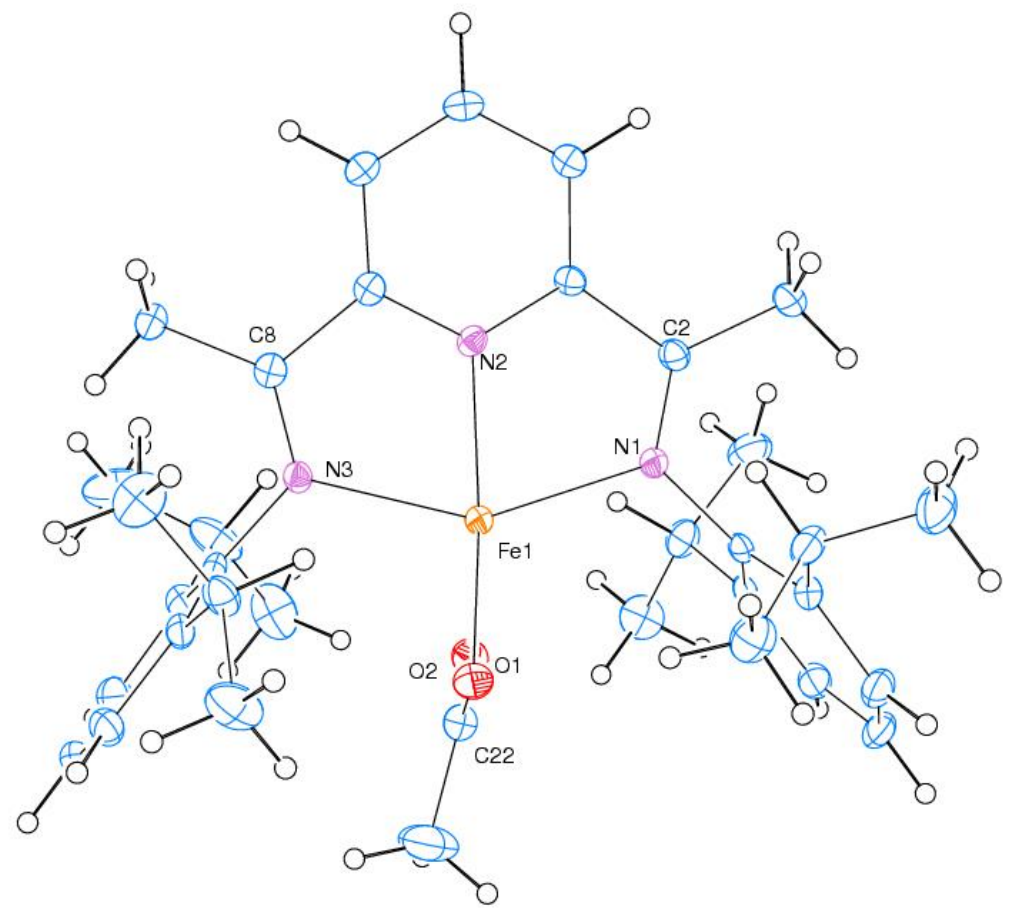

Figure S-27. Molecular structure of [(PDI)FeOAc $]^{+}$

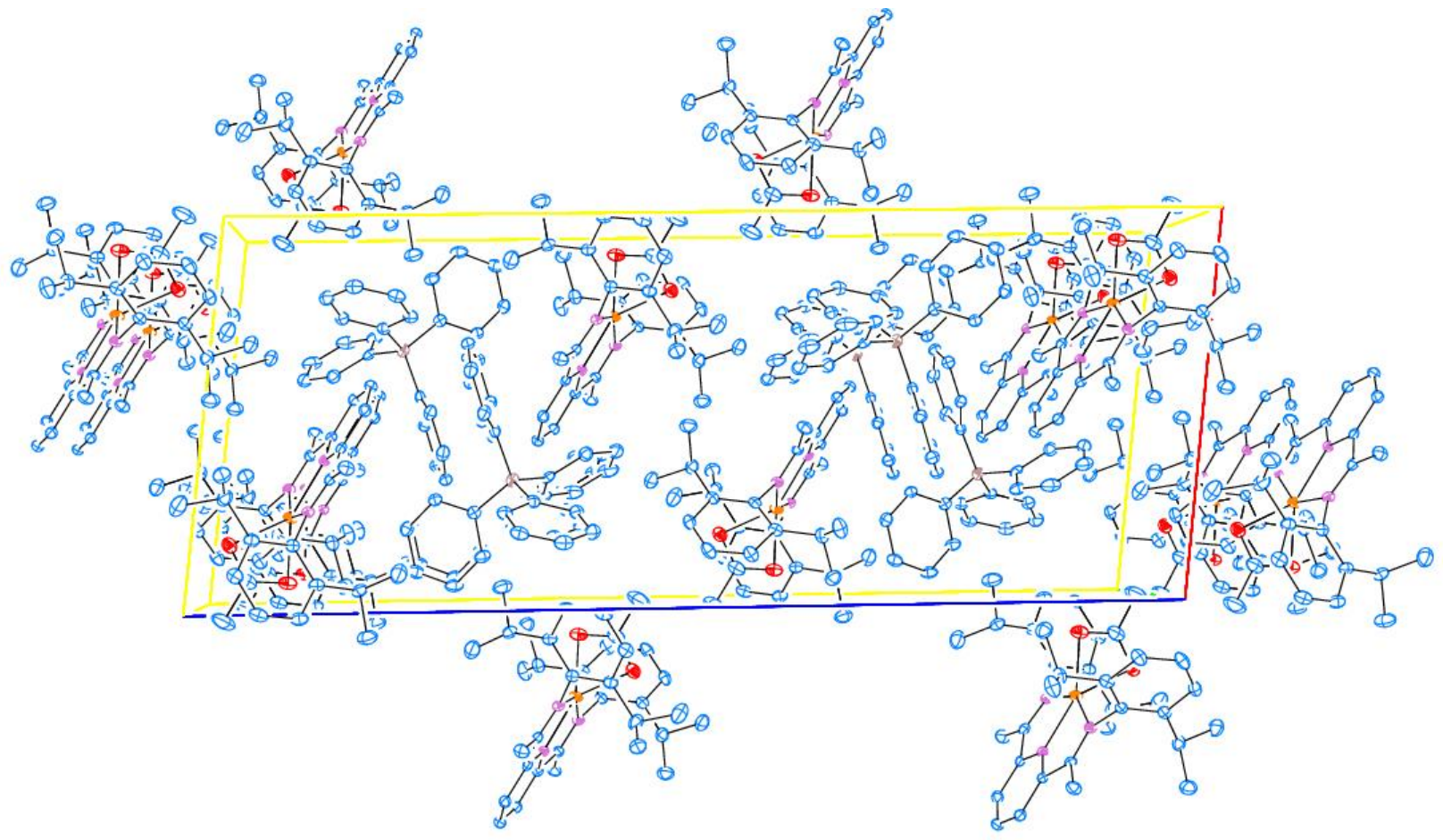

Figure S-28. Unit cell of 4. 
Table S-11. Atomic coordinates ( $\times 10^{4}$ ) and equivalent isotropic displacement parameters $\left(\AA^{2} \times 10^{3}\right)$

for 4. $\mathrm{U}(\mathrm{eq})$ is defined as one third of the trace of the orthogonalized $\mathrm{Uij}^{\mathrm{ij}}$ tensor.

\begin{tabular}{|c|c|c|c|c|}
\hline & $\mathrm{X}$ & $\mathrm{y}$ & $\mathrm{z}$ & $\mathrm{U}(\mathrm{eq})$ \\
\hline $\mathrm{Fe}(1)$ & $7573(1)$ & $4417(1)$ & $5972(1)$ & $24(1)$ \\
\hline $\mathrm{N}(1)$ & $6865(2)$ & $5967(2)$ & $5900(1)$ & $22(1)$ \\
\hline $\mathrm{N}(2)$ & $6156(2)$ & $4328(2)$ & $6253(1)$ & $21(1)$ \\
\hline $\mathrm{N}(3)$ & $7434(2)$ & $2777(2)$ & $6161(1)$ & $22(1)$ \\
\hline $\mathrm{O}(1)$ & $8235(2)$ & $4166(2)$ & $5404(1)$ & $37(1)$ \\
\hline $\mathrm{O}(2)$ & $9203(2)$ & $4532(2)$ & $6006(1)$ & $39(1)$ \\
\hline $\mathrm{B}(1)$ & $6717(3)$ & $3755(2)$ & $8118(1)$ & $26(1)$ \\
\hline $\mathrm{C}(1)$ & $5455(2)$ & $7179(2)$ & $6091(1)$ & $33(1)$ \\
\hline$C(2)$ & $6013(2)$ & $6144(2)$ & $6077(1)$ & $23(1)$ \\
\hline$C(3)$ & $5568(2)$ & $5203(2)$ & $6281(1)$ & $21(1)$ \\
\hline $\mathrm{C}(4)$ & $4632(2)$ & $5180(2)$ & $6471(1)$ & $25(1)$ \\
\hline$C(5)$ & $4324(2)$ & $4225(2)$ & $6634(1)$ & $28(1)$ \\
\hline$C(6)$ & $4940(2)$ & $3327(2)$ & $6608(1)$ & $26(1)$ \\
\hline$C(7)$ & $5873(2)$ & $3404(2)$ & $6413(1)$ & $21(1)$ \\
\hline $\mathrm{C}(8)$ & $6627(2)$ & $2521(2)$ & $6350(1)$ & $23(1)$ \\
\hline $\mathrm{C}(9)$ & $6373(2)$ & $1445(2)$ & $6505(1)$ & $33(1)$ \\
\hline$C(10)$ & $6762(3)$ & $5444(3)$ & $4598(1)$ & $46(1)$ \\
\hline$C(11)$ & $5355(2)$ & $6506(3)$ & $4905(1)$ & $47(1)$ \\
\hline$C(12)$ & $6427(2)$ & $5944(2)$ & $4996(1)$ & $33(1)$ \\
\hline$C(13)$ & $7251(2)$ & $6674(2)$ & $5224(1)$ & $25(1)$ \\
\hline$C(14)$ & $7872(2)$ & $7328(2)$ & $4996(1)$ & $32(1)$ \\
\hline$C(15)$ & $8596(2)$ & $8023(2)$ & $5201(1)$ & $37(1)$ \\
\hline$C(16)$ & $8705(2)$ & $8100(2)$ & $5636(1)$ & $35(1)$ \\
\hline$C(17)$ & $8097(2)$ & $7477(2)$ & $5883(1)$ & $28(1)$ \\
\hline $\mathrm{C}(18)$ & $7392(2)$ & $6756(2)$ & $5667(1)$ & $22(1)$ \\
\hline C(19) & $8263(2)$ & $7592(2)$ & $6362(1)$ & $32(1)$ \\
\hline $\mathrm{C}(20)$ & $8062(3)$ & $8727(2)$ & $6495(1)$ & $48(1)$ \\
\hline $\mathrm{C}(21)$ & $9376(2)$ & $7221(3)$ & $6536(1)$ & $44(1)$ \\
\hline$C(22)$ & $9142(3)$ & $4247(2)$ & $5615(1)$ & $41(1)$ \\
\hline $\mathrm{C}(23)$ & 10121(3) & $3963(4)$ & $5426(1)$ & $77(1)$ \\
\hline $\mathrm{C}(24)$ & $7230(3)$ & $1902(3)$ & 4933(1) & $60(1)$ \\
\hline$C(25)$ & $6505(3)$ & 445(4) & $5355(1)$ & $70(1)$ \\
\hline$C(26)$ & $7049(3)$ & $1525(3)$ & $5376(1)$ & $50(1)$ \\
\hline$C(27)$ & $8085(2)$ & $1486(2)$ & $5673(1)$ & $31(1)$ \\
\hline $\mathrm{C}(28)$ & $8924(2)$ & $862(2)$ & $5564(1)$ & $35(1)$ \\
\hline $\mathrm{C}(29)$ & $9863(2)$ & $772(2)$ & $5826(1)$ & $34(1)$ \\
\hline$C(30)$ & $9994(2)$ & $1317(2)$ & $6205(1)$ & $34(1)$ \\
\hline $\mathrm{C}(31)$ & $9199(2)$ & $1975(2)$ & $6328(1)$ & $27(1)$ \\
\hline$C(32)$ & $8236(2)$ & $2029(2)$ & $6060(1)$ & $22(1)$ \\
\hline $\mathrm{C}(33)$ & $9367(2)$ & $2599(2)$ & $6738(1)$ & $32(1)$ \\
\hline
\end{tabular}




\begin{tabular}{lrrrl}
$\mathrm{C}(34)$ & $10528(2)$ & $2916(3)$ & $6856(1)$ & $53(1)$ \\
$\mathrm{C}(35)$ & $8956(3)$ & $2018(3)$ & $7104(1)$ & $53(1)$ \\
$\mathrm{C}(36)$ & $7506(2)$ & $4168(2)$ & $7774(1)$ & $24(1)$ \\
$\mathrm{C}(37)$ & $7179(2)$ & $4596(2)$ & $7376(1)$ & $26(1)$ \\
$\mathrm{C}(38)$ & $7902(2)$ & $5032(2)$ & $7123(1)$ & $31(1)$ \\
$\mathrm{C}(39)$ & $8982(2)$ & $5041(2)$ & $7255(1)$ & $35(1)$ \\
$\mathrm{C}(40)$ & $9332(2)$ & $4614(2)$ & $7648(1)$ & $36(1)$ \\
$\mathrm{C}(41)$ & $8609(2)$ & $4198(2)$ & $7899(1)$ & $32(1)$ \\
$\mathrm{C}(42)$ & $7250(2)$ & $2661(2)$ & $8332(1)$ & $26(1)$ \\
$\mathrm{C}(43)$ & $7522(2)$ & $1837(2)$ & $8066(1)$ & $30(1)$ \\
$\mathrm{C}(44)$ & $8004(2)$ & $904(2)$ & $8220(1)$ & $35(1)$ \\
$\mathrm{C}(45)$ & $8243(2)$ & $758(2)$ & $8650(1)$ & $38(1)$ \\
$\mathrm{C}(46)$ & $8008(2)$ & $1548(2)$ & $8923(1)$ & $38(1)$ \\
$\mathrm{C}(47)$ & $7523(2)$ & $2480(2)$ & $8764(1)$ & $30(1)$ \\
$\mathrm{C}(48)$ & $6658(2)$ & $4699(2)$ & $8473(1)$ & $27(1)$ \\
$\mathrm{C}(49)$ & $6087(2)$ & $4564(2)$ & $8822(1)$ & $34(1)$ \\
$\mathrm{C}(50)$ & $6020(2)$ & $5335(3)$ & $9129(1)$ & $46(1)$ \\
$\mathrm{C}(51)$ & $6499(3)$ & $6297(3)$ & $9087(1)$ & $55(1)$ \\
$\mathrm{C}(52)$ & $7047(2)$ & $6482(3)$ & $8745(1)$ & $51(1)$ \\
$\mathrm{C}(53)$ & $7132(2)$ & $5703(2)$ & $8446(1)$ & $35(1)$ \\
$\mathrm{C}(54)$ & $5487(2)$ & $3540(2)$ & $7895(1)$ & $25(1)$ \\
$\mathrm{C}(55)$ & $4767(2)$ & $4381(2)$ & $7825(1)$ & $29(1)$ \\
$\mathrm{C}(56)$ & $3709(2)$ & $4251(3)$ & $7658(1)$ & $37(1)$ \\
$\mathrm{C}(57)$ & $3327(2)$ & $3254(3)$ & $7547(1)$ & $38(1)$ \\
$\mathrm{C}(58)$ & $4010(2)$ & $2404(3)$ & $7601(1)$ & $37(1)$ \\
$\mathrm{C}(59)$ & $5061(2)$ & $2551(2)$ & $7771(1)$ & $31(1)$ \\
& & & & \\
\hline
\end{tabular}


Table S-12. Bond lengths $[\AA ̊]$ and angles $\left[{ }^{\circ}\right]$ for 4.

\begin{tabular}{ll}
\hline $\mathrm{Fe}(1)-\mathrm{O}(2)$ & $2.0462(19)$ \\
$\mathrm{Fe}(1)-\mathrm{N}(2)$ & $2.082(2)$ \\
$\mathrm{Fe}(1)-\mathrm{O}(1)$ & $2.0869(19)$ \\
$\mathrm{Fe}(1)-\mathrm{N}(1)$ & $2.153(2)$ \\
$\mathrm{Fe}(1)-\mathrm{N}(3)$ & $2.169(2)$ \\
$\mathrm{N}(1)-\mathrm{C}(2)$ & $1.282(3)$ \\
$\mathrm{N}(1)-\mathrm{C}(18)$ & $1.446(3)$ \\
$\mathrm{N}(2)-\mathrm{C}(7)$ & $1.336(3)$ \\
$\mathrm{N}(2)-\mathrm{C}(3)$ & $1.338(3)$ \\
$\mathrm{N}(3)-\mathrm{C}(8)$ & $1.276(3)$ \\
$\mathrm{N}(3)-\mathrm{C}(32)$ & $1.445(3)$ \\
$\mathrm{O}(1)-\mathrm{C}(22)$ & $1.258(4)$ \\
$\mathrm{O}(2)-\mathrm{C}(22)$ & $1.282(4)$ \\
$\mathrm{B}(1)-\mathrm{C}(36)$ & $1.639(4)$ \\
$\mathrm{B}(1)-\mathrm{C}(48)$ & $1.647(4)$ \\
$\mathrm{B}(1)-\mathrm{C}(54)$ & $1.648(4)$ \\
$\mathrm{B}(1)-\mathrm{C}(42)$ & $1.649(4)$ \\
$\mathrm{C}(1)-\mathrm{C}(2)$ & $1.488(4)$ \\
$\mathrm{C}(1)-\mathrm{H}(1 \mathrm{~A})$ & 0.9800 \\
$\mathrm{C}(1)-\mathrm{H}(1 \mathrm{~B})$ & 0.9800 \\
$\mathrm{C}(1)-\mathrm{H}(1 \mathrm{C})$ & 0.9800 \\
$\mathrm{C}(2)-\mathrm{C}(3)$ & $1.492(4)$ \\
$\mathrm{C}(3)-\mathrm{C}(4)$ & $1.382(4)$ \\
$\mathrm{C}(4)-\mathrm{C}(5)$ & $1.384(4)$ \\
$\mathrm{C}(4)-\mathrm{H}(4)$ & 0.9500 \\
$\mathrm{C}(5)-\mathrm{C}(6)$ & $1.381(4)$ \\
$\mathrm{C}(5)-\mathrm{H}(5)$ & 0.9500 \\
$\mathrm{C}(6)-\mathrm{C}(7)$ & $1.390(4)$ \\
$\mathrm{C}(6)-\mathrm{H}(6)$ & 0.9500 \\
$\mathrm{C}(7)-\mathrm{C}(8)$ & $1.493(4)$ \\
$\mathrm{C}(8)-\mathrm{C}(9)$ & $1.491(4)$ \\
$\mathrm{C}(9)-\mathrm{H}(9 \mathrm{~A})$ & 0.9800 \\
$\mathrm{C}(9)-\mathrm{H}(9 \mathrm{~B})$ & 0.9800 \\
$\mathrm{C}(9)-\mathrm{H}(9 \mathrm{C})$ & 0.9800 \\
$\mathrm{C}(10)-\mathrm{C}(12)$ & $1.512(4)$ \\
$\mathrm{C}(10)-\mathrm{H}(10 \mathrm{~A})$ & 0.9800 \\
$\mathrm{C}(10)-\mathrm{H}(10 \mathrm{~B})$ & 0.9800 \\
$\mathrm{C}(10)-\mathrm{H}(10 \mathrm{C})$ & 0.9800 \\
$\mathrm{C}(11)-\mathrm{C}(12)$ & $1.522(4)$ \\
$\mathrm{C}(11)-\mathrm{H}(11 \mathrm{~A})$ & 0.9800 \\
$\mathrm{C}(11)-\mathrm{H}(11 \mathrm{~B})$ & 0.9800 \\
$\mathrm{C}(11)-\mathrm{H}(11 \mathrm{C})$ & 0.9800 \\
$\mathrm{C}(12)-\mathrm{C}(13)$ & $1.509(4)$ \\
&
\end{tabular}

$\begin{array}{ll}\mathrm{C}(12)-\mathrm{H}(12) & 1.0000 \\ \mathrm{C}(13)-\mathrm{C}(14) & 1.392(4) \\ \mathrm{C}(13)-\mathrm{C}(18) & 1.396(4) \\ \mathrm{C}(14)-\mathrm{C}(15) & 1.375(4) \\ \mathrm{C}(14)-\mathrm{H}(14) & 0.9500 \\ \mathrm{C}(15)-\mathrm{C}(16) & 1.369(4) \\ \mathrm{C}(15)-\mathrm{H}(15) & 0.9500 \\ \mathrm{C}(16)-\mathrm{C}(17) & 1.398(4) \\ \mathrm{C}(16)-\mathrm{H}(16) & 0.9500 \\ \mathrm{C}(17)-\mathrm{C}(18) & 1.397(4) \\ \mathrm{C}(17)-\mathrm{C}(19) & 1.513(4) \\ \mathrm{C}(19)-\mathrm{C}(21) & 1.519(4) \\ \mathrm{C}(19)-\mathrm{C}(20) & 1.525(4) \\ \mathrm{C}(19)-\mathrm{H}(19) & 1.0000 \\ \mathrm{C}(20)-\mathrm{H}(20 \mathrm{~A}) & 0.9800 \\ \mathrm{C}(20)-\mathrm{H}(20 \mathrm{~B}) & 0.9800 \\ \mathrm{C}(20)-\mathrm{H}(20 \mathrm{C}) & 0.9800 \\ \mathrm{C}(21)-\mathrm{H}(21 \mathrm{~A}) & 0.9800 \\ \mathrm{C}(21)-\mathrm{H}(21 \mathrm{~B}) & 0.9800 \\ \mathrm{C}(21)-\mathrm{H}(21 \mathrm{C}) & 0.9800 \\ \mathrm{C}(22)-\mathrm{C}(23) & 1.472(4) \\ \mathrm{C}(23)-\mathrm{H}(23 \mathrm{~A}) & 0.9800 \\ \mathrm{C}(23)-\mathrm{H}(23 \mathrm{~B}) & 0.9800 \\ \mathrm{C}(23)-\mathrm{H}(23 \mathrm{C}) & 0.9800 \\ \mathrm{C}(24)-\mathrm{C}(26) & 1.523(5) \\ \mathrm{C}(24)-\mathrm{H}(24 \mathrm{~A}) & 0.9800 \\ \mathrm{C}(24)-\mathrm{H}(24 \mathrm{~B}) & 0.9800 \\ \mathrm{C}(24)-\mathrm{H}(24 \mathrm{C}) & 0.9800 \\ \mathrm{C}(25)-\mathrm{C}(26) & 1.525(5) \\ \mathrm{C}(25)-\mathrm{H}(25 \mathrm{~A}) & 0.9800 \\ \mathrm{C}(25)-\mathrm{H}(25 \mathrm{~B}) & 0.9800 \\ \mathrm{C}(25)-\mathrm{H}(25 \mathrm{C}) & 0.9800 \\ \mathrm{C}(26)-\mathrm{C}(27) & 1.518(4) \\ \mathrm{C}(26)-\mathrm{H}(26) & 1.0000 \\ \mathrm{C}(27)-\mathrm{C}(28) & 1.392(4) \\ \mathrm{C}(27)-\mathrm{C}(32) & 1.396(4) \\ \mathrm{C}(28)-\mathrm{C}(29) & 1.368(4) \\ \mathrm{C}(28)-\mathrm{H}(28) & 0.9500 \\ \mathrm{C}(29)-\mathrm{C}(30) & 1.376(4) \\ \mathrm{C}(29)-\mathrm{H}(29) & 0.9500 \\ \mathrm{C}(30)-\mathrm{C}(31) & 1.390(4) \\ \mathrm{C}(30)-\mathrm{H}(30) & 0.9500 \\ \mathrm{C}(31)-\mathrm{C}(32) & 1.401(4) \\ \mathrm{C}(31)-\mathrm{C}(33) & 1.512(4) \\ \mathrm{C}(33)-\mathrm{C}(35) & 1.509(4) \\ \mathrm{C}(33)-\mathrm{C}(34) & 1.520(4) \\ & \end{array}$




\begin{tabular}{|c|c|c|c|}
\hline $\mathrm{C}(33)-\mathrm{H}(33)$ & 1.0000 & $\mathrm{C}(57)-\mathrm{C}(58)$ & $1.374(4)$ \\
\hline $\mathrm{C}(34)-\mathrm{H}(34 \mathrm{~A})$ & 0.9800 & $\mathrm{C}(57)-\mathrm{H}(57)$ & 0.9500 \\
\hline $\mathrm{C}(34)-\mathrm{H}(34 \mathrm{~B})$ & 0.9800 & $\mathrm{C}(58)-\mathrm{C}(59)$ & $1.382(4)$ \\
\hline $\mathrm{C}(34)-\mathrm{H}(34 \mathrm{C})$ & 0.9800 & $\mathrm{C}(58)-\mathrm{H}(58)$ & 0.9500 \\
\hline $\mathrm{C}(35)-\mathrm{H}(35 \mathrm{~A})$ & 0.9800 & $\mathrm{C}(59)-\mathrm{H}(59)$ & 0.9500 \\
\hline $\mathrm{C}(35)-\mathrm{H}(35 \mathrm{~B})$ & 0.9800 & & \\
\hline $\mathrm{C}(35)-\mathrm{H}(35 \mathrm{C})$ & 0.9800 & $\mathrm{O}(2)-\mathrm{Fe}(1)-\mathrm{N}(2)$ & 151.91(9) \\
\hline $\mathrm{C}(36)-\mathrm{C}(37)$ & $1.390(4)$ & $\mathrm{O}(2)-\mathrm{Fe}(1)-\mathrm{O}(1)$ & $63.94(9)$ \\
\hline$C(36)-C(41)$ & $1.399(4)$ & $\mathrm{N}(2)-\mathrm{Fe}(1)-\mathrm{O}(1)$ & $143.44(9)$ \\
\hline $\mathrm{C}(37)-\mathrm{C}(38)$ & $1.390(4)$ & $\mathrm{O}(2)-\mathrm{Fe}(1)-\mathrm{N}(1)$ & $109.93(8)$ \\
\hline $\mathrm{C}(37)-\mathrm{H}(37)$ & 0.9500 & $\mathrm{~N}(2)-\mathrm{Fe}(1)-\mathrm{N}(1)$ & $74.36(8)$ \\
\hline $\mathrm{C}(38)-\mathrm{C}(39)$ & $1.375(4)$ & $\mathrm{O}(1)-\mathrm{Fe}(1)-\mathrm{N}(1)$ & $104.35(8)$ \\
\hline $\mathrm{C}(38)-\mathrm{H}(38)$ & 0.9500 & $\mathrm{O}(2)-\mathrm{Fe}(1)-\mathrm{N}(3)$ & $99.47(8)$ \\
\hline $\mathrm{C}(39)-\mathrm{C}(40)$ & $1.382(4)$ & $\mathrm{N}(2)-\mathrm{Fe}(1)-\mathrm{N}(3)$ & $74.41(8)$ \\
\hline $\mathrm{C}(39)-\mathrm{H}(39)$ & 0.9500 & $\mathrm{O}(1)-\mathrm{Fe}(1)-\mathrm{N}(3)$ & $98.36(8)$ \\
\hline $\mathrm{C}(40)-\mathrm{C}(41)$ & $1.376(4)$ & $\mathrm{N}(1)-\mathrm{Fe}(1)-\mathrm{N}(3)$ & $148.53(8)$ \\
\hline $\mathrm{C}(40)-\mathrm{H}(40)$ & 0.9500 & $\mathrm{C}(2)-\mathrm{N}(1)-\mathrm{C}(18)$ & $123.7(2)$ \\
\hline $\mathrm{C}(41)-\mathrm{H}(41)$ & 0.9500 & $\mathrm{C}(2)-\mathrm{N}(1)-\mathrm{Fe}(1)$ & $117.98(17)$ \\
\hline$C(42)-C(47)$ & $1.389(4)$ & $\mathrm{C}(18)-\mathrm{N}(1)-\mathrm{Fe}(1)$ & $118.25(15)$ \\
\hline$C(42)-C(43)$ & $1.405(4)$ & $\mathrm{C}(7)-\mathrm{N}(2)-\mathrm{C}(3)$ & $121.6(2)$ \\
\hline$C(43)-C(44)$ & $1.388(4)$ & $\mathrm{C}(7)-\mathrm{N}(2)-\mathrm{Fe}(1)$ & $119.08(17)$ \\
\hline $\mathrm{C}(43)-\mathrm{H}(43)$ & 0.9500 & $\mathrm{C}(3)-\mathrm{N}(2)-\mathrm{Fe}(1)$ & $119.27(17)$ \\
\hline $\mathrm{C}(44)-\mathrm{C}(45)$ & $1.372(4)$ & $\mathrm{C}(8)-\mathrm{N}(3)-\mathrm{C}(32)$ & $123.4(2)$ \\
\hline $\mathrm{C}(44)-\mathrm{H}(44)$ & 0.9500 & $\mathrm{C}(8)-\mathrm{N}(3)-\mathrm{Fe}(1)$ & $117.69(18)$ \\
\hline$C(45)-C(46)$ & $1.376(4)$ & $\mathrm{C}(32)-\mathrm{N}(3)-\mathrm{Fe}(1)$ & $118.94(16)$ \\
\hline $\mathrm{C}(45)-\mathrm{H}(45)$ & 0.9500 & $\mathrm{C}(22)-\mathrm{O}(1)-\mathrm{Fe}(1)$ & $87.62(19)$ \\
\hline$C(46)-C(47)$ & $1.393(4)$ & $\mathrm{C}(22)-\mathrm{O}(2)-\mathrm{Fe}(1)$ & $88.78(18)$ \\
\hline $\mathrm{C}(46)-\mathrm{H}(46)$ & 0.9500 & $\mathrm{C}(36)-\mathrm{B}(1)-\mathrm{C}(48)$ & $107.5(2)$ \\
\hline $\mathrm{C}(47)-\mathrm{H}(47)$ & 0.9500 & $\mathrm{C}(36)-\mathrm{B}(1)-\mathrm{C}(54)$ & $112.2(2)$ \\
\hline $\mathrm{C}(48)-\mathrm{C}(49)$ & $1.393(4)$ & $\mathrm{C}(48)-\mathrm{B}(1)-\mathrm{C}(54)$ & $107.4(2)$ \\
\hline $\mathrm{C}(48)-\mathrm{C}(53)$ & $1.408(4)$ & $\mathrm{C}(36)-\mathrm{B}(1)-\mathrm{C}(42)$ & $106.9(2)$ \\
\hline $\mathrm{C}(49)-\mathrm{C}(50)$ & $1.384(4)$ & $\mathrm{C}(48)-\mathrm{B}(1)-\mathrm{C}(42)$ & $112.0(2)$ \\
\hline $\mathrm{C}(49)-\mathrm{H}(49)$ & 0.9500 & $\mathrm{C}(54)-\mathrm{B}(1)-\mathrm{C}(42)$ & $110.8(2)$ \\
\hline $\mathrm{C}(50)-\mathrm{C}(51)$ & $1.370(5)$ & $\mathrm{C}(2)-\mathrm{C}(1)-\mathrm{H}(1 \mathrm{~A})$ & 109.5 \\
\hline $\mathrm{C}(50)-\mathrm{H}(50)$ & 0.9500 & $\mathrm{C}(2)-\mathrm{C}(1)-\mathrm{H}(1 \mathrm{~B})$ & 109.5 \\
\hline $\mathrm{C}(51)-\mathrm{C}(52)$ & $1.365(5)$ & $\mathrm{H}(1 \mathrm{~A})-\mathrm{C}(1)-\mathrm{H}(1 \mathrm{~B})$ & 109.5 \\
\hline $\mathrm{C}(51)-\mathrm{H}(51)$ & 0.9500 & $\mathrm{C}(2)-\mathrm{C}(1)-\mathrm{H}(1 \mathrm{C})$ & 109.5 \\
\hline $\mathrm{C}(52)-\mathrm{C}(53)$ & $1.378(4)$ & $\mathrm{H}(1 \mathrm{~A})-\mathrm{C}(1)-\mathrm{H}(1 \mathrm{C})$ & 109.5 \\
\hline $\mathrm{C}(52)-\mathrm{H}(52)$ & 0.9500 & $\mathrm{H}(1 \mathrm{~B})-\mathrm{C}(1)-\mathrm{H}(1 \mathrm{C})$ & 109.5 \\
\hline $\mathrm{C}(53)-\mathrm{H}(53)$ & 0.9500 & $\mathrm{~N}(1)-\mathrm{C}(2)-\mathrm{C}(1)$ & $126.0(2)$ \\
\hline $\mathrm{C}(54)-\mathrm{C}(59)$ & $1.398(4)$ & $\mathrm{N}(1)-\mathrm{C}(2)-\mathrm{C}(3)$ & $114.9(2)$ \\
\hline $\mathrm{C}(54)-\mathrm{C}(55)$ & $1.398(4)$ & $\mathrm{C}(1)-\mathrm{C}(2)-\mathrm{C}(3)$ & 119.1(2) \\
\hline$C(55)-C(56)$ & $1.384(4)$ & $\mathrm{N}(2)-\mathrm{C}(3)-\mathrm{C}(4)$ & $121.0(2)$ \\
\hline $\mathrm{C}(55)-\mathrm{H}(55)$ & 0.9500 & $\mathrm{~N}(2)-\mathrm{C}(3)-\mathrm{C}(2)$ & $113.2(2)$ \\
\hline $\mathrm{C}(56)-\mathrm{C}(57)$ & $1.381(4)$ & $\mathrm{C}(4)-\mathrm{C}(3)-\mathrm{C}(2)$ & $125.8(2)$ \\
\hline $\mathrm{C}(56)-\mathrm{H}(56)$ & 0.9500 & $C(3)-C(4)-C(5)$ & $117.9(2)$ \\
\hline
\end{tabular}




\begin{tabular}{|c|c|c|c|}
\hline $\mathrm{C}(3)-\mathrm{C}(4)-\mathrm{H}(4)$ & 121.0 & $\mathrm{C}(14)-\mathrm{C}(15)-\mathrm{H}(15)$ & 119.8 \\
\hline $\mathrm{C}(5)-\mathrm{C}(4)-\mathrm{H}(4)$ & 121.0 & $C(15)-C(16)-C(17)$ & $121.6(3)$ \\
\hline $\mathrm{C}(6)-\mathrm{C}(5)-\mathrm{C}(4)$ & $120.8(2)$ & $\mathrm{C}(15)-\mathrm{C}(16)-\mathrm{H}(16)$ & 119.2 \\
\hline $\mathrm{C}(6)-\mathrm{C}(5)-\mathrm{H}(5)$ & 119.6 & $\mathrm{C}(17)-\mathrm{C}(16)-\mathrm{H}(16)$ & 119.2 \\
\hline $\mathrm{C}(4)-\mathrm{C}(5)-\mathrm{H}(5)$ & 119.6 & $C(18)-C(17)-C(16)$ & $116.7(3)$ \\
\hline$C(5)-C(6)-C(7)$ & $118.4(2)$ & $C(18)-C(17)-C(19)$ & $124.2(2)$ \\
\hline $\mathrm{C}(5)-\mathrm{C}(6)-\mathrm{H}(6)$ & 120.8 & $C(16)-C(17)-C(19)$ & $119.0(3)$ \\
\hline $\mathrm{C}(7)-\mathrm{C}(6)-\mathrm{H}(6)$ & 120.8 & $\mathrm{C}(13)-\mathrm{C}(18)-\mathrm{C}(17)$ & $122.9(2)$ \\
\hline $\mathrm{N}(2)-\mathrm{C}(7)-\mathrm{C}(6)$ & $120.3(2)$ & $\mathrm{C}(13)-\mathrm{C}(18)-\mathrm{N}(1)$ & $116.7(2)$ \\
\hline $\mathrm{N}(2)-\mathrm{C}(7)-\mathrm{C}(8)$ & $113.8(2)$ & $\mathrm{C}(17)-\mathrm{C}(18)-\mathrm{N}(1)$ & $120.1(2)$ \\
\hline $\mathrm{C}(6)-\mathrm{C}(7)-\mathrm{C}(8)$ & $126.0(2)$ & $\mathrm{C}(17)-\mathrm{C}(19)-\mathrm{C}(21)$ & $110.5(2)$ \\
\hline $\mathrm{N}(3)-\mathrm{C}(8)-\mathrm{C}(9)$ & $126.6(2)$ & $C(17)-C(19)-C(20)$ & $111.0(2)$ \\
\hline $\mathrm{N}(3)-\mathrm{C}(8)-\mathrm{C}(7)$ & $115.0(2)$ & $C(21)-C(19)-C(20)$ & $111.4(3)$ \\
\hline $\mathrm{C}(9)-\mathrm{C}(8)-\mathrm{C}(7)$ & $118.4(2)$ & $\mathrm{C}(17)-\mathrm{C}(19)-\mathrm{H}(19)$ & 107.9 \\
\hline $\mathrm{C}(8)-\mathrm{C}(9)-\mathrm{H}(9 \mathrm{~A})$ & 109.5 & $\mathrm{C}(21)-\mathrm{C}(19)-\mathrm{H}(19)$ & 107.9 \\
\hline $\mathrm{C}(8)-\mathrm{C}(9)-\mathrm{H}(9 \mathrm{~B})$ & 109.5 & $\mathrm{C}(20)-\mathrm{C}(19)-\mathrm{H}(19)$ & 107.9 \\
\hline $\mathrm{H}(9 \mathrm{~A})-\mathrm{C}(9)-\mathrm{H}(9 \mathrm{~B})$ & 109.5 & $\mathrm{C}(19)-\mathrm{C}(20)-\mathrm{H}(20 \mathrm{~A})$ & 109.5 \\
\hline $\mathrm{C}(8)-\mathrm{C}(9)-\mathrm{H}(9 \mathrm{C})$ & 109.5 & $\mathrm{C}(19)-\mathrm{C}(20)-\mathrm{H}(20 \mathrm{~B})$ & 109.5 \\
\hline $\mathrm{H}(9 \mathrm{~A})-\mathrm{C}(9)-\mathrm{H}(9 \mathrm{C})$ & 109.5 & $\mathrm{H}(20 \mathrm{~A})-\mathrm{C}(20)-\mathrm{H}(20 \mathrm{~B})$ & 109.5 \\
\hline $\mathrm{H}(9 \mathrm{~B})-\mathrm{C}(9)-\mathrm{H}(9 \mathrm{C})$ & 109.5 & $\mathrm{C}(19)-\mathrm{C}(20)-\mathrm{H}(20 \mathrm{C})$ & 109.5 \\
\hline $\mathrm{C}(12)-\mathrm{C}(10)-\mathrm{H}(10 \mathrm{~A})$ & 109.5 & $\mathrm{H}(20 \mathrm{~A})-\mathrm{C}(20)-\mathrm{H}(20 \mathrm{C})$ & 109.5 \\
\hline $\mathrm{C}(12)-\mathrm{C}(10)-\mathrm{H}(10 \mathrm{~B})$ & 109.5 & $\mathrm{H}(20 \mathrm{~B})-\mathrm{C}(20)-\mathrm{H}(20 \mathrm{C})$ & 109.5 \\
\hline $\mathrm{H}(10 \mathrm{~A})-\mathrm{C}(10)-\mathrm{H}(10 \mathrm{~B})$ & 109.5 & $\mathrm{C}(19)-\mathrm{C}(21)-\mathrm{H}(21 \mathrm{~A})$ & 109.5 \\
\hline $\mathrm{C}(12)-\mathrm{C}(10)-\mathrm{H}(10 \mathrm{C})$ & 109.5 & $\mathrm{C}(19)-\mathrm{C}(21)-\mathrm{H}(21 \mathrm{~B})$ & 109.5 \\
\hline $\mathrm{H}(10 \mathrm{~A})-\mathrm{C}(10)-\mathrm{H}(10 \mathrm{C})$ & 109.5 & $\mathrm{H}(21 \mathrm{~A})-\mathrm{C}(21)-\mathrm{H}(21 \mathrm{~B})$ & 109.5 \\
\hline $\mathrm{H}(10 \mathrm{~B})-\mathrm{C}(10)-\mathrm{H}(10 \mathrm{C})$ & 109.5 & $\mathrm{C}(19)-\mathrm{C}(21)-\mathrm{H}(21 \mathrm{C})$ & 109.5 \\
\hline $\mathrm{C}(12)-\mathrm{C}(11)-\mathrm{H}(11 \mathrm{~A})$ & 109.5 & $\mathrm{H}(21 \mathrm{~A})-\mathrm{C}(21)-\mathrm{H}(21 \mathrm{C})$ & 109.5 \\
\hline $\mathrm{C}(12)-\mathrm{C}(11)-\mathrm{H}(11 \mathrm{~B})$ & 109.5 & $\mathrm{H}(21 \mathrm{~B})-\mathrm{C}(21)-\mathrm{H}(21 \mathrm{C})$ & 109.5 \\
\hline $\mathrm{H}(11 \mathrm{~A})-\mathrm{C}(11)-\mathrm{H}(11 \mathrm{~B})$ & 109.5 & $\mathrm{O}(1)-\mathrm{C}(22)-\mathrm{O}(2)$ & $119.1(3)$ \\
\hline $\mathrm{C}(12)-\mathrm{C}(11)-\mathrm{H}(11 \mathrm{C})$ & 109.5 & $\mathrm{O}(1)-\mathrm{C}(22)-\mathrm{C}(23)$ & $121.0(3)$ \\
\hline $\mathrm{H}(11 \mathrm{~A})-\mathrm{C}(11)-\mathrm{H}(11 \mathrm{C})$ & 109.5 & $\mathrm{O}(2)-\mathrm{C}(22)-\mathrm{C}(23)$ & $119.8(3)$ \\
\hline $\mathrm{H}(11 \mathrm{~B})-\mathrm{C}(11)-\mathrm{H}(11 \mathrm{C})$ & 109.5 & $\mathrm{C}(22)-\mathrm{C}(23)-\mathrm{H}(23 \mathrm{~A})$ & 109.5 \\
\hline $\mathrm{C}(13)-\mathrm{C}(12)-\mathrm{C}(10)$ & $114.3(2)$ & $\mathrm{C}(22)-\mathrm{C}(23)-\mathrm{H}(23 \mathrm{~B})$ & 109.5 \\
\hline $\mathrm{C}(13)-\mathrm{C}(12)-\mathrm{C}(11)$ & $110.5(2)$ & $\mathrm{H}(23 \mathrm{~A})-\mathrm{C}(23)-\mathrm{H}(23 \mathrm{~B})$ & 109.5 \\
\hline $\mathrm{C}(10)-\mathrm{C}(12)-\mathrm{C}(11)$ & 111.1(3) & $\mathrm{C}(22)-\mathrm{C}(23)-\mathrm{H}(23 \mathrm{C})$ & 109.5 \\
\hline $\mathrm{C}(13)-\mathrm{C}(12)-\mathrm{H}(12)$ & 106.8 & $\mathrm{H}(23 \mathrm{~A})-\mathrm{C}(23)-\mathrm{H}(23 \mathrm{C})$ & 109.5 \\
\hline $\mathrm{C}(10)-\mathrm{C}(12)-\mathrm{H}(12)$ & 106.8 & $\mathrm{H}(23 \mathrm{~B})-\mathrm{C}(23)-\mathrm{H}(23 \mathrm{C})$ & 109.5 \\
\hline $\mathrm{C}(11)-\mathrm{C}(12)-\mathrm{H}(12)$ & 106.8 & $\mathrm{C}(26)-\mathrm{C}(24)-\mathrm{H}(24 \mathrm{~A})$ & 109.5 \\
\hline $\mathrm{C}(14)-\mathrm{C}(13)-\mathrm{C}(18)$ & $117.4(3)$ & $\mathrm{C}(26)-\mathrm{C}(24)-\mathrm{H}(24 \mathrm{~B})$ & 109.5 \\
\hline $\mathrm{C}(14)-\mathrm{C}(13)-\mathrm{C}(12)$ & $120.7(3)$ & $\mathrm{H}(24 \mathrm{~A})-\mathrm{C}(24)-\mathrm{H}(24 \mathrm{~B})$ & 109.5 \\
\hline $\mathrm{C}(18)-\mathrm{C}(13)-\mathrm{C}(12)$ & $121.8(2)$ & $\mathrm{C}(26)-\mathrm{C}(24)-\mathrm{H}(24 \mathrm{C})$ & 109.5 \\
\hline$C(15)-C(14)-C(13)$ & $121.0(3)$ & $\mathrm{H}(24 \mathrm{~A})-\mathrm{C}(24)-\mathrm{H}(24 \mathrm{C})$ & 109.5 \\
\hline $\mathrm{C}(15)-\mathrm{C}(14)-\mathrm{H}(14)$ & 119.5 & $\mathrm{H}(24 \mathrm{~B})-\mathrm{C}(24)-\mathrm{H}(24 \mathrm{C})$ & 109.5 \\
\hline $\mathrm{C}(13)-\mathrm{C}(14)-\mathrm{H}(14)$ & 119.5 & $\mathrm{C}(26)-\mathrm{C}(25)-\mathrm{H}(25 \mathrm{~A})$ & 109.5 \\
\hline $\mathrm{C}(16)-\mathrm{C}(15)-\mathrm{C}(14)$ & $120.4(3)$ & $\mathrm{C}(26)-\mathrm{C}(25)-\mathrm{H}(25 \mathrm{~B})$ & 109.5 \\
\hline $\mathrm{C}(16)-\mathrm{C}(15)-\mathrm{H}(15)$ & 119.8 & $\mathrm{H}(25 \mathrm{~A})-\mathrm{C}(25)-\mathrm{H}(25 \mathrm{~B})$ & 109.5 \\
\hline
\end{tabular}




\begin{tabular}{|c|c|c|c|}
\hline $\mathrm{C}(26)-\mathrm{C}(25)-\mathrm{H}(25 \mathrm{C})$ & 109.5 & $\mathrm{C}(37)-\mathrm{C}(36)-\mathrm{B}(1)$ & $125.9(2)$ \\
\hline $\mathrm{H}(25 \mathrm{~A})-\mathrm{C}(25)-\mathrm{H}(25 \mathrm{C})$ & 109.5 & $\mathrm{C}(41)-\mathrm{C}(36)-\mathrm{B}(1)$ & $118.4(2)$ \\
\hline $\mathrm{H}(25 \mathrm{~B})-\mathrm{C}(25)-\mathrm{H}(25 \mathrm{C})$ & 109.5 & $\mathrm{C}(36)-\mathrm{C}(37)-\mathrm{C}(38)$ & $122.0(3)$ \\
\hline$C(27)-C(26)-C(24)$ & $112.0(3)$ & $\mathrm{C}(36)-\mathrm{C}(37)-\mathrm{H}(37)$ & 119.0 \\
\hline$C(27)-C(26)-C(25)$ & $110.3(3)$ & $\mathrm{C}(38)-\mathrm{C}(37)-\mathrm{H}(37)$ & 119.0 \\
\hline$C(24)-C(26)-C(25)$ & $110.6(3)$ & $\mathrm{C}(39)-\mathrm{C}(38)-\mathrm{C}(37)$ & $121.0(3)$ \\
\hline $\mathrm{C}(27)-\mathrm{C}(26)-\mathrm{H}(26)$ & 107.9 & $\mathrm{C}(39)-\mathrm{C}(38)-\mathrm{H}(38)$ & 119.5 \\
\hline $\mathrm{C}(24)-\mathrm{C}(26)-\mathrm{H}(26)$ & 107.9 & $\mathrm{C}(37)-\mathrm{C}(38)-\mathrm{H}(38)$ & 119.5 \\
\hline $\mathrm{C}(25)-\mathrm{C}(26)-\mathrm{H}(26)$ & 107.9 & $\mathrm{C}(38)-\mathrm{C}(39)-\mathrm{C}(40)$ & $118.3(3)$ \\
\hline $\mathrm{C}(28)-\mathrm{C}(27)-\mathrm{C}(32)$ & $117.6(3)$ & $\mathrm{C}(38)-\mathrm{C}(39)-\mathrm{H}(39)$ & 120.8 \\
\hline $\mathrm{C}(28)-\mathrm{C}(27)-\mathrm{C}(26)$ & 119.1(3) & $\mathrm{C}(40)-\mathrm{C}(39)-\mathrm{H}(39)$ & 120.8 \\
\hline$C(32)-C(27)-C(26)$ & $123.3(3)$ & $\mathrm{C}(41)-\mathrm{C}(40)-\mathrm{C}(39)$ & $120.2(3)$ \\
\hline$C(29)-C(28)-C(27)$ & $121.6(3)$ & $\mathrm{C}(41)-\mathrm{C}(40)-\mathrm{H}(40)$ & 119.9 \\
\hline $\mathrm{C}(29)-\mathrm{C}(28)-\mathrm{H}(28)$ & 119.2 & $\mathrm{C}(39)-\mathrm{C}(40)-\mathrm{H}(40)$ & 119.9 \\
\hline $\mathrm{C}(27)-\mathrm{C}(28)-\mathrm{H}(28)$ & 119.2 & $\mathrm{C}(40)-\mathrm{C}(41)-\mathrm{C}(36)$ & $123.1(3)$ \\
\hline $\mathrm{C}(28)-\mathrm{C}(29)-\mathrm{C}(30)$ & $119.8(3)$ & $\mathrm{C}(40)-\mathrm{C}(41)-\mathrm{H}(41)$ & 118.4 \\
\hline $\mathrm{C}(28)-\mathrm{C}(29)-\mathrm{H}(29)$ & 120.1 & $\mathrm{C}(36)-\mathrm{C}(41)-\mathrm{H}(41)$ & 118.4 \\
\hline $\mathrm{C}(30)-\mathrm{C}(29)-\mathrm{H}(29)$ & 120.1 & $\mathrm{C}(47)-\mathrm{C}(42)-\mathrm{C}(43)$ & $114.6(3)$ \\
\hline$C(29)-C(30)-C(31)$ & $121.7(3)$ & $\mathrm{C}(47)-\mathrm{C}(42)-\mathrm{B}(1)$ & $125.9(2)$ \\
\hline $\mathrm{C}(29)-\mathrm{C}(30)-\mathrm{H}(30)$ & 119.2 & $\mathrm{C}(43)-\mathrm{C}(42)-\mathrm{B}(1)$ & $119.4(2)$ \\
\hline $\mathrm{C}(31)-\mathrm{C}(30)-\mathrm{H}(30)$ & 119.2 & $\mathrm{C}(44)-\mathrm{C}(43)-\mathrm{C}(42)$ & $123.0(3)$ \\
\hline$C(30)-C(31)-C(32)$ & $117.3(3)$ & $\mathrm{C}(44)-\mathrm{C}(43)-\mathrm{H}(43)$ & 118.5 \\
\hline$C(30)-C(31)-C(33)$ & $121.2(2)$ & $\mathrm{C}(42)-\mathrm{C}(43)-\mathrm{H}(43)$ & 118.5 \\
\hline$C(32)-C(31)-C(33)$ & $121.5(2)$ & $\mathrm{C}(45)-\mathrm{C}(44)-\mathrm{C}(43)$ & $120.1(3)$ \\
\hline$C(27)-C(32)-C(31)$ & $122.0(2)$ & $\mathrm{C}(45)-\mathrm{C}(44)-\mathrm{H}(44)$ & 120.0 \\
\hline $\mathrm{C}(27)-\mathrm{C}(32)-\mathrm{N}(3)$ & $119.2(2)$ & $\mathrm{C}(43)-\mathrm{C}(44)-\mathrm{H}(44)$ & 120.0 \\
\hline$C(31)-C(32)-N(3)$ & $118.3(2)$ & $\mathrm{C}(44)-\mathrm{C}(45)-\mathrm{C}(46)$ & 119.1(3) \\
\hline$C(35)-C(33)-C(31)$ & 112.1(3) & $\mathrm{C}(44)-\mathrm{C}(45)-\mathrm{H}(45)$ & 120.5 \\
\hline$C(35)-C(33)-C(34)$ & $110.0(3)$ & $\mathrm{C}(46)-\mathrm{C}(45)-\mathrm{H}(45)$ & 120.5 \\
\hline$C(31)-C(33)-C(34)$ & $112.7(2)$ & $\mathrm{C}(45)-\mathrm{C}(46)-\mathrm{C}(47)$ & $120.2(3)$ \\
\hline $\mathrm{C}(35)-\mathrm{C}(33)-\mathrm{H}(33)$ & 107.2 & $\mathrm{C}(45)-\mathrm{C}(46)-\mathrm{H}(46)$ & 119.9 \\
\hline $\mathrm{C}(31)-\mathrm{C}(33)-\mathrm{H}(33)$ & 107.2 & $\mathrm{C}(47)-\mathrm{C}(46)-\mathrm{H}(46)$ & 119.9 \\
\hline $\mathrm{C}(34)-\mathrm{C}(33)-\mathrm{H}(33)$ & 107.2 & $\mathrm{C}(42)-\mathrm{C}(47)-\mathrm{C}(46)$ & $123.0(3)$ \\
\hline $\mathrm{C}(33)-\mathrm{C}(34)-\mathrm{H}(34 \mathrm{~A})$ & 109.5 & $\mathrm{C}(42)-\mathrm{C}(47)-\mathrm{H}(47)$ & 118.5 \\
\hline $\mathrm{C}(33)-\mathrm{C}(34)-\mathrm{H}(34 \mathrm{~B})$ & 109.5 & $\mathrm{C}(46)-\mathrm{C}(47)-\mathrm{H}(47)$ & 118.5 \\
\hline $\mathrm{H}(34 \mathrm{~A})-\mathrm{C}(34)-\mathrm{H}(34 \mathrm{~B})$ & 109.5 & $\mathrm{C}(49)-\mathrm{C}(48)-\mathrm{C}(53)$ & $114.7(3)$ \\
\hline $\mathrm{C}(33)-\mathrm{C}(34)-\mathrm{H}(34 \mathrm{C})$ & 109.5 & $\mathrm{C}(49)-\mathrm{C}(48)-\mathrm{B}(1)$ & $121.3(3)$ \\
\hline $\mathrm{H}(34 \mathrm{~A})-\mathrm{C}(34)-\mathrm{H}(34 \mathrm{C})$ & 109.5 & $\mathrm{C}(53)-\mathrm{C}(48)-\mathrm{B}(1)$ & $124.0(3)$ \\
\hline $\mathrm{H}(34 \mathrm{~B})-\mathrm{C}(34)-\mathrm{H}(34 \mathrm{C})$ & 109.5 & $\mathrm{C}(50)-\mathrm{C}(49)-\mathrm{C}(48)$ & $123.2(3)$ \\
\hline $\mathrm{C}(33)-\mathrm{C}(35)-\mathrm{H}(35 \mathrm{~A})$ & 109.5 & $\mathrm{C}(50)-\mathrm{C}(49)-\mathrm{H}(49)$ & 118.4 \\
\hline $\mathrm{C}(33)-\mathrm{C}(35)-\mathrm{H}(35 \mathrm{~B})$ & 109.5 & $\mathrm{C}(48)-\mathrm{C}(49)-\mathrm{H}(49)$ & 118.4 \\
\hline $\mathrm{H}(35 \mathrm{~A})-\mathrm{C}(35)-\mathrm{H}(35 \mathrm{~B})$ & 109.5 & $\mathrm{C}(51)-\mathrm{C}(50)-\mathrm{C}(49)$ & $119.7(3)$ \\
\hline $\mathrm{C}(33)-\mathrm{C}(35)-\mathrm{H}(35 \mathrm{C})$ & 109.5 & $\mathrm{C}(51)-\mathrm{C}(50)-\mathrm{H}(50)$ & 120.2 \\
\hline $\mathrm{H}(35 \mathrm{~A})-\mathrm{C}(35)-\mathrm{H}(35 \mathrm{C})$ & 109.5 & $\mathrm{C}(49)-\mathrm{C}(50)-\mathrm{H}(50)$ & 120.2 \\
\hline $\mathrm{H}(35 \mathrm{~B})-\mathrm{C}(35)-\mathrm{H}(35 \mathrm{C})$ & 109.5 & $\mathrm{C}(52)-\mathrm{C}(51)-\mathrm{C}(50)$ & $119.6(3)$ \\
\hline$C(37)-C(36)-C(41)$ & $115.3(2)$ & $\mathrm{C}(52)-\mathrm{C}(51)-\mathrm{H}(51)$ & 120.2 \\
\hline
\end{tabular}




$\begin{array}{ll}\mathrm{C}(50)-\mathrm{C}(51)-\mathrm{H}(51) & 120.2 \\ \mathrm{C}(51)-\mathrm{C}(52)-\mathrm{C}(53) & 120.5(3) \\ \mathrm{C}(51)-\mathrm{C}(52)-\mathrm{H}(52) & 119.8 \\ \mathrm{C}(53)-\mathrm{C}(52)-\mathrm{H}(52) & 119.8 \\ \mathrm{C}(52)-\mathrm{C}(53)-\mathrm{C}(48) & 122.4(3) \\ \mathrm{C}(52)-\mathrm{C}(53)-\mathrm{H}(53) & 118.8 \\ \mathrm{C}(48)-\mathrm{C}(53)-\mathrm{H}(53) & 118.8 \\ \mathrm{C}(59)-\mathrm{C}(54)-\mathrm{C}(55) & 114.5(3) \\ \mathrm{C}(59)-\mathrm{C}(54)-\mathrm{B}(1) & 125.4(2) \\ \mathrm{C}(55)-\mathrm{C}(54)-\mathrm{B}(1) & 120.1(2) \\ \mathrm{C}(56)-\mathrm{C}(55)-\mathrm{C}(54) & 123.2(3) \\ \mathrm{C}(56)-\mathrm{C}(55)-\mathrm{H}(55) & 118.4 \\ \mathrm{C}(54)-\mathrm{C}(55)-\mathrm{H}(55) & 118.4 \\ \mathrm{C}(57)-\mathrm{C}(56)-\mathrm{C}(55) & 119.9(3) \\ \mathrm{C}(57)-\mathrm{C}(56)-\mathrm{H}(56) & 120.1 \\ \mathrm{C}(55)-\mathrm{C}(56)-\mathrm{H}(56) & 120.1 \\ \mathrm{C}(58)-\mathrm{C}(57)-\mathrm{C}(56) & 119.1(3) \\ \mathrm{C}(58)-\mathrm{C}(57)-\mathrm{H}(57) & 120.4 \\ \mathrm{C}(56)-\mathrm{C}(57)-\mathrm{H}(57) & 120.4 \\ \mathrm{C}(57)-\mathrm{C}(58)-\mathrm{C}(59) & 120.1(3) \\ \mathrm{C}(57)-\mathrm{C}(58)-\mathrm{H}(58) & 120.0 \\ \mathrm{C}(59)-\mathrm{C}(58)-\mathrm{H}(58) & 120.0 \\ \mathrm{C}(58)-\mathrm{C}(59)-\mathrm{C}(54) & 123.2(3) \\ \mathrm{C}(58)-\mathrm{C}(59)-\mathrm{H}(59) & 118.4 \\ \mathrm{C}(54)-\mathrm{C}(59)-\mathrm{H}(59) & 118.4 \\ & \end{array}$


Table S-13. Anisotropic displacement parameters $\left(\AA^{2} \times 10^{3}\right)$ for 4 . The anisotropic displacement factor exponent takes the form: $-2 \pi^{2}\left[h^{2} a^{* 2} U^{11}+\ldots+2 h k a^{*} b^{*} U^{12}\right]$

\begin{tabular}{|c|c|c|c|c|c|c|}
\hline & $\mathrm{U}^{11}$ & $\mathrm{U}^{22}$ & $\mathrm{U}^{33}$ & $\mathrm{U}^{23}$ & $\mathrm{U}^{13}$ & $\mathrm{U}^{12}$ \\
\hline$\overline{\mathrm{Fe}(1)}$ & $20(1)$ & 19(1) & $34(1)$ & $4(1)$ & $6(1)$ & $2(1)$ \\
\hline $\mathrm{N}(1)$ & $20(1)$ & $20(1)$ & $26(1)$ & $3(1)$ & $3(1)$ & 1(1) \\
\hline $\mathrm{N}(2)$ & $19(1)$ & $22(1)$ & $21(1)$ & $4(1)$ & $0(1)$ & $-1(1)$ \\
\hline $\mathrm{N}(3)$ & $19(1)$ & 19(1) & $25(1)$ & 1(1) & $0(1)$ & $0(1)$ \\
\hline $\mathrm{O}(1)$ & $39(1)$ & $35(1)$ & $38(1)$ & $4(1)$ & 11(1) & $6(1)$ \\
\hline $\mathrm{O}(2)$ & $26(1)$ & $36(1)$ & $56(2)$ & 2(1) & $7(1)$ & $-3(1)$ \\
\hline $\mathrm{B}(1)$ & $30(2)$ & $22(2)$ & $25(2)$ & $0(1)$ & $0(1)$ & $-1(1)$ \\
\hline $\mathrm{C}(1)$ & $31(2)$ & $29(2)$ & $41(2)$ & $7(1)$ & 11(1) & $9(1)$ \\
\hline $\mathrm{C}(2)$ & $21(2)$ & $24(2)$ & $24(2)$ & $2(1)$ & 1(1) & $3(1)$ \\
\hline $\mathrm{C}(3)$ & $18(1)$ & $24(2)$ & 19(1) & 1(1) & $-1(1)$ & 2(1) \\
\hline $\mathrm{C}(4)$ & $23(2)$ & $28(2)$ & $22(2)$ & $-1(1)$ & $0(1)$ & $2(1)$ \\
\hline $\mathrm{C}(5)$ & $22(2)$ & $37(2)$ & $25(2)$ & $-2(1)$ & $6(1)$ & $-4(1)$ \\
\hline$C(6)$ & $27(2)$ & $28(2)$ & $23(2)$ & $2(1)$ & $2(1)$ & $-4(1)$ \\
\hline $\mathrm{C}(7)$ & $22(1)$ & $24(2)$ & $16(1)$ & $2(1)$ & $-4(1)$ & $-1(1)$ \\
\hline $\mathrm{C}(8)$ & $22(2)$ & $25(2)$ & $20(1)$ & $0(1)$ & $-4(1)$ & $-2(1)$ \\
\hline $\mathrm{C}(9)$ & $32(2)$ & $26(2)$ & $41(2)$ & $8(1)$ & $8(1)$ & $-1(1)$ \\
\hline $\mathrm{C}(10)$ & $49(2)$ & $52(2)$ & $36(2)$ & $-8(2)$ & $-3(2)$ & $-3(2)$ \\
\hline $\mathrm{C}(11)$ & $32(2)$ & $54(2)$ & $55(2)$ & 11(2) & $5(2)$ & $-6(2)$ \\
\hline $\mathrm{C}(12)$ & $38(2)$ & $31(2)$ & $30(2)$ & $7(1)$ & 2(1) & $-2(1)$ \\
\hline $\mathrm{C}(13)$ & $27(2)$ & 18(1) & $31(2)$ & $3(1)$ & $7(1)$ & $8(1)$ \\
\hline $\mathrm{C}(14)$ & $41(2)$ & $29(2)$ & $29(2)$ & $4(1)$ & 11(1) & $5(2)$ \\
\hline $\mathrm{C}(15)$ & $47(2)$ & $26(2)$ & $41(2)$ & $1(2)$ & $21(2)$ & $-8(2)$ \\
\hline $\mathrm{C}(16)$ & $43(2)$ & $24(2)$ & $40(2)$ & $-4(1)$ & $14(2)$ & $-8(1)$ \\
\hline $\mathrm{C}(17)$ & $29(2)$ & 17(1) & $37(2)$ & $1(1)$ & $8(1)$ & $2(1)$ \\
\hline $\mathrm{C}(18)$ & $23(2)$ & 18(1) & $28(2)$ & $7(1)$ & $7(1)$ & $8(1)$ \\
\hline $\mathrm{C}(19)$ & $40(2)$ & $26(2)$ & $31(2)$ & 1(1) & $9(1)$ & $-9(1)$ \\
\hline $\mathrm{C}(20)$ & 72(2) & $32(2)$ & $43(2)$ & $-6(2)$ & $20(2)$ & $-8(2)$ \\
\hline $\mathrm{C}(21)$ & $41(2)$ & $44(2)$ & $45(2)$ & $-1(2)$ & $-2(2)$ & $-8(2)$ \\
\hline $\mathrm{C}(22)$ & $39(2)$ & $30(2)$ & $57(2)$ & 12(2) & $18(2)$ & $5(2)$ \\
\hline $\mathrm{C}(23)$ & $47(2)$ & $95(3)$ & $95(3)$ & $15(3)$ & $37(2)$ & $18(2)$ \\
\hline $\mathrm{C}(24)$ & $69(3)$ & $61(2)$ & $46(2)$ & $-8(2)$ & $-13(2)$ & $14(2)$ \\
\hline $\mathrm{C}(25)$ & $40(2)$ & $119(4)$ & $49(2)$ & $-20(2)$ & $3(2)$ & $-26(2)$ \\
\hline $\mathrm{C}(26)$ & $41(2)$ & 69(3) & $37(2)$ & $-23(2)$ & $-5(2)$ & $19(2)$ \\
\hline $\mathrm{C}(27)$ & $28(2)$ & $27(2)$ & $36(2)$ & 1(1) & $4(1)$ & $1(1)$ \\
\hline $\mathrm{C}(28)$ & $37(2)$ & $27(2)$ & $41(2)$ & $-6(1)$ & $8(2)$ & $-2(1)$ \\
\hline $\mathrm{C}(29)$ & $29(2)$ & 21(2) & $55(2)$ & $1(2)$ & $13(2)$ & 1(1) \\
\hline $\mathrm{C}(30)$ & $26(2)$ & $27(2)$ & $47(2)$ & $1(2)$ & $1(1)$ & 2(1) \\
\hline $\mathrm{C}(31)$ & $24(2)$ & 19(1) & $37(2)$ & $5(1)$ & $2(1)$ & $2(1)$ \\
\hline $\mathrm{C}(32)$ & $24(2)$ & 14(1) & $29(2)$ & $5(1)$ & $4(1)$ & $-2(1)$ \\
\hline $\mathrm{C}(33)$ & $26(2)$ & $32(2)$ & $37(2)$ & $0(1)$ & $-4(1)$ & $6(1)$ \\
\hline $\mathrm{C}(34)$ & $32(2)$ & $60(2)$ & $65(2)$ & $-22(2)$ & $-4(2)$ & $-1(2)$ \\
\hline
\end{tabular}




\begin{tabular}{llllccc}
$\mathrm{C}(35)$ & $59(2)$ & $58(2)$ & $41(2)$ & $2(2)$ & $-1(2)$ & $-11(2)$ \\
$\mathrm{C}(36)$ & $27(2)$ & $13(1)$ & $30(2)$ & $1(1)$ & $1(1)$ & $0(1)$ \\
$\mathrm{C}(37)$ & $25(2)$ & $22(2)$ & $30(2)$ & $-3(1)$ & $1(1)$ & $-4(1)$ \\
$\mathrm{C}(38)$ & $37(2)$ & $27(2)$ & $28(2)$ & $4(1)$ & $1(1)$ & $-1(1)$ \\
$\mathrm{C}(39)$ & $31(2)$ & $30(2)$ & $45(2)$ & $6(2)$ & $10(2)$ & $-4(1)$ \\
$\mathrm{C}(40)$ & $24(2)$ & $28(2)$ & $55(2)$ & $12(2)$ & $1(2)$ & $1(1)$ \\
$\mathrm{C}(41)$ & $26(2)$ & $29(2)$ & $41(2)$ & $13(1)$ & $-2(1)$ & $-1(1)$ \\
$\mathrm{C}(42)$ & $24(2)$ & $22(2)$ & $32(2)$ & $3(1)$ & $5(1)$ & $-4(1)$ \\
$\mathrm{C}(43)$ & $29(2)$ & $25(2)$ & $37(2)$ & $3(1)$ & $5(1)$ & $-2(1)$ \\
$\mathrm{C}(44)$ & $29(2)$ & $23(2)$ & $53(2)$ & $-1(2)$ & $10(2)$ & $-3(1)$ \\
$\mathrm{C}(45)$ & $32(2)$ & $26(2)$ & $57(2)$ & $15(2)$ & $4(2)$ & $1(1)$ \\
$\mathrm{C}(46)$ & $36(2)$ & $38(2)$ & $39(2)$ & $15(2)$ & $0(2)$ & $1(2)$ \\
$\mathrm{C}(47)$ & $30(2)$ & $27(2)$ & $33(2)$ & $5(1)$ & $4(1)$ & $-2(1)$ \\
$\mathrm{C}(48)$ & $23(2)$ & $27(2)$ & $28(2)$ & $2(1)$ & $-8(1)$ & $5(1)$ \\
$\mathrm{C}(49)$ & $34(2)$ & $35(2)$ & $33(2)$ & $-1(2)$ & $1(1)$ & $3(1)$ \\
$\mathrm{C}(50)$ & $34(2)$ & $65(3)$ & $38(2)$ & $-10(2)$ & $1(2)$ & $16(2)$ \\
$\mathrm{C}(51)$ & $32(2)$ & $60(3)$ & $70(3)$ & $-39(2)$ & $-6(2)$ & $6(2)$ \\
$\mathrm{C}(52)$ & $30(2)$ & $35(2)$ & $85(3)$ & $-26(2)$ & $-1(2)$ & $0(2)$ \\
$\mathrm{C}(53)$ & $28(2)$ & $27(2)$ & $49(2)$ & $-3(2)$ & $-3(1)$ & $-2(1)$ \\
$\mathrm{C}(54)$ & $30(2)$ & $27(2)$ & $19(1)$ & $2(1)$ & $6(1)$ & $-3(1)$ \\
$\mathrm{C}(55)$ & $27(2)$ & $32(2)$ & $27(2)$ & $-6(1)$ & $2(1)$ & $-2(1)$ \\
$\mathrm{C}(56)$ & $29(2)$ & $51(2)$ & $32(2)$ & $-7(2)$ & $2(1)$ & $2(2)$ \\
$\mathrm{C}(57)$ & $24(2)$ & $62(2)$ & $27(2)$ & $-4(2)$ & $1(1)$ & $-13(2)$ \\
$\mathrm{C}(58)$ & $44(2)$ & $39(2)$ & $28(2)$ & $2(2)$ & $1(1)$ & $-20(2)$ \\
$\mathrm{C}(59)$ & $39(2)$ & $26(2)$ & $27(2)$ & $4(1)$ & $0(1)$ & $-9(1)$ \\
& & & & & & \\
\hline & & & & & & \\
\end{tabular}


Table S-14. Hydrogen coordinates ( $\times 10^{4}$ ) and isotropic displacement parameters $\left(\AA^{2} \times 10^{3}\right)$ for 4 .

\begin{tabular}{|c|c|c|c|c|}
\hline & $\mathrm{X}$ & $\mathrm{y}$ & $\mathrm{z}$ & $\mathrm{U}(\mathrm{eq})$ \\
\hline $\mathrm{H}(1 \mathrm{~A})$ & 5519 & 7438 & 6385 & 49 \\
\hline $\mathrm{H}(1 \mathrm{~B})$ & 4697 & 7091 & 5986 & 49 \\
\hline $\mathrm{H}(1 \mathrm{C})$ & 5781 & 7691 & 5912 & 49 \\
\hline $\mathrm{H}(4)$ & 4213 & 5800 & 6490 & 30 \\
\hline $\mathrm{H}(5)$ & 3681 & 4187 & 6765 & 33 \\
\hline $\mathrm{H}(6)$ & 4731 & 2673 & 6721 & 31 \\
\hline $\mathrm{H}(9 \mathrm{~A})$ & 6949 & 955 & 6455 & 49 \\
\hline $\mathrm{H}(9 \mathrm{~B})$ & 5698 & 1198 & 6351 & 49 \\
\hline $\mathrm{H}(9 \mathrm{C})$ & 6303 & 1476 & 6810 & 49 \\
\hline $\mathrm{H}(10 \mathrm{~A})$ & 6800 & 5990 & 4380 & 69 \\
\hline $\mathrm{H}(10 \mathrm{~B})$ & 6237 & 4906 & 4492 & 69 \\
\hline $\mathrm{H}(10 \mathrm{C})$ & 7466 & 5114 & 4663 & 69 \\
\hline $\mathrm{H}(11 \mathrm{~A})$ & 5441 & 7135 & 4730 & 71 \\
\hline $\mathrm{H}(11 \mathrm{~B})$ & 5099 & 6720 & 5174 & 71 \\
\hline $\mathrm{H}(11 \mathrm{C})$ & 4834 & 6026 & 4752 & 71 \\
\hline $\mathrm{H}(12)$ & 6319 & 5352 & 5197 & 40 \\
\hline $\mathrm{H}(14)$ & 7794 & 7293 & 4694 & 39 \\
\hline $\mathrm{H}(15)$ & 9023 & 8453 & 5041 & 44 \\
\hline $\mathrm{H}(16)$ & 9205 & 8589 & 5772 & 42 \\
\hline H(19) & 7732 & 7126 & 6485 & 38 \\
\hline $\mathrm{H}(20 \mathrm{~A})$ & 8579 & 9199 & 6382 & 72 \\
\hline $\mathrm{H}(20 \mathrm{~B})$ & 8142 & 8774 & 6807 & 72 \\
\hline $\mathrm{H}(20 \mathrm{C})$ & 7334 & 8938 & 6383 & 72 \\
\hline $\mathrm{H}(21 \mathrm{~A})$ & 9472 & 6483 & 6455 & 66 \\
\hline $\mathrm{H}(21 \mathrm{~B})$ & 9462 & 7280 & 6847 & 66 \\
\hline $\mathrm{H}(21 \mathrm{C})$ & 9913 & 7663 & 6419 & 66 \\
\hline $\mathrm{H}(23 \mathrm{~A})$ & 9933 & 3758 & 5128 & 116 \\
\hline $\mathrm{H}(23 \mathrm{~B})$ & 10475 & 3369 & 5584 & 116 \\
\hline $\mathrm{H}(23 \mathrm{C})$ & 10605 & 4572 & 5440 & 116 \\
\hline $\mathrm{H}(24 \mathrm{~A})$ & 7533 & 2617 & 4950 & 90 \\
\hline $\mathrm{H}(24 \mathrm{~B})$ & 6545 & 1911 & 4751 & 90 \\
\hline $\mathrm{H}(24 \mathrm{C})$ & 7726 & 1421 & 4812 & 90 \\
\hline $\mathrm{H}(25 \mathrm{~A})$ & 6951 & -66 & 5222 & 105 \\
\hline $\mathrm{H}(25 \mathrm{~B})$ & 5805 & 499 & 5186 & 105 \\
\hline $\mathrm{H}(25 \mathrm{C})$ & 6410 & 208 & 5643 & 105 \\
\hline $\mathrm{H}(26)$ & 6560 & 2040 & 5496 & 60 \\
\hline $\mathrm{H}(28)$ & 8844 & 490 & 5301 & 42 \\
\hline $\mathrm{H}(29)$ & 10423 & 336 & 5746 & 41 \\
\hline $\mathrm{H}(30)$ & 10644 & 1241 & 6387 & 40 \\
\hline $\mathrm{H}(33)$ & 8941 & 3266 & 6693 & 39 \\
\hline
\end{tabular}




\begin{tabular}{lrrrl}
$\mathrm{H}(34 \mathrm{~A})$ & 10580 & 3394 & 7101 & 79 \\
$\mathrm{H}(34 \mathrm{~B})$ & 10794 & 3275 & 6614 & 79 \\
$\mathrm{H}(34 \mathrm{C})$ & 10959 & 2282 & 6929 & 79 \\
$\mathrm{H}(35 \mathrm{~A})$ & 8188 & 1882 & 7037 & 80 \\
$\mathrm{H}(35 \mathrm{~B})$ & 9075 & 2451 & 7362 & 80 \\
$\mathrm{H}(35 \mathrm{C})$ & 9337 & 1344 & 7150 & 80 \\
$\mathrm{H}(37)$ & 6439 & 4592 & 7273 & 31 \\
$\mathrm{H}(38)$ & 7645 & 5328 & 6855 & 37 \\
$\mathrm{H}(39)$ & 9474 & 5334 & 7081 & 42 \\
$\mathrm{H}(40)$ & 10075 & 4607 & 7746 & 43 \\
$\mathrm{H}(41)$ & 8870 & 3917 & 8169 & 39 \\
$\mathrm{H}(43)$ & 7369 & 1922 & 7767 & 36 \\
$\mathrm{H}(44)$ & 8167 & 367 & 8027 & 41 \\
$\mathrm{H}(45)$ & 8568 & 119 & 8757 & 46 \\
$\mathrm{H}(46)$ & 8176 & 1460 & 9221 & 46 \\
$\mathrm{H}(47)$ & 7372 & 3015 & 8959 & 36 \\
$\mathrm{H}(49)$ & 5727 & 3912 & 8851 & 41 \\
$\mathrm{H}(50)$ & 5644 & 5197 & 9367 & 55 \\
$\mathrm{H}(51)$ & 6451 & 6833 & 9295 & 66 \\
$\mathrm{H}(52)$ & 7372 & 7151 & 8714 & 61 \\
$\mathrm{H}(53)$ & 7525 & 5848 & 8214 & 42 \\
$\mathrm{H}(55)$ & 5015 & 5076 & 7896 & 35 \\
$\mathrm{H}(56)$ & 3248 & 4847 & 7620 & 45 \\
$\mathrm{H}(57)$ & 2601 & 3156 & 7434 & 45 \\
$\mathrm{H}(58)$ & 3759 & 1715 & 7522 & 45 \\
$\mathrm{H}(59)$ & 5517 & 1950 & 7806 & 38 \\
& & & & \\
\hline
\end{tabular}


Table S-15. Torsion angles $\left[{ }^{\circ}\right]$ for 4.

\begin{tabular}{lc}
\hline $\mathrm{C}(18)-\mathrm{N}(1)-\mathrm{C}(2)-\mathrm{C}(1)$ & $-3.0(4)$ \\
$\mathrm{Fe}(1)-\mathrm{N}(1)-\mathrm{C}(2)-\mathrm{C}(1)$ & $175.1(2)$ \\
$\mathrm{C}(18)-\mathrm{N}(1)-\mathrm{C}(2)-\mathrm{C}(3)$ & $176.7(2)$ \\
$\mathrm{Fe}(1)-\mathrm{N}(1)-\mathrm{C}(2)-\mathrm{C}(3)$ & $-5.1(3)$ \\
$\mathrm{C}(7)-\mathrm{N}(2)-\mathrm{C}(3)-\mathrm{C}(4)$ & $-1.0(4)$ \\
$\mathrm{Fe}(1)-\mathrm{N}(2)-\mathrm{C}(3)-\mathrm{C}(4)$ & $-178.93(19)$ \\
$\mathrm{C}(7)-\mathrm{N}(2)-\mathrm{C}(3)-\mathrm{C}(2)$ & $-179.1(2)$ \\
$\mathrm{Fe}(1)-\mathrm{N}(2)-\mathrm{C}(3)-\mathrm{C}(2)$ & $3.0(3)$ \\
$\mathrm{N}(1)-\mathrm{C}(2)-\mathrm{C}(3)-\mathrm{N}(2)$ & $1.5(3)$ \\
$\mathrm{C}(1)-\mathrm{C}(2)-\mathrm{C}(3)-\mathrm{N}(2)$ & $-178.7(2)$ \\
$\mathrm{N}(1)-\mathrm{C}(2)-\mathrm{C}(3)-\mathrm{C}(4)$ & $-176.5(2)$ \\
$\mathrm{C}(1)-\mathrm{C}(2)-\mathrm{C}(3)-\mathrm{C}(4)$ & $3.3(4)$ \\
$\mathrm{N}(2)-\mathrm{C}(3)-\mathrm{C}(4)-\mathrm{C}(5)$ & $0.3(4)$ \\
$\mathrm{C}(2)-\mathrm{C}(3)-\mathrm{C}(4)-\mathrm{C}(5)$ & $178.1(2)$ \\
$\mathrm{C}(3)-\mathrm{C}(4)-\mathrm{C}(5)-\mathrm{C}(6)$ & $0.4(4)$ \\
$\mathrm{C}(4)-\mathrm{C}(5)-\mathrm{C}(6)-\mathrm{C}(7)$ & $-0.4(4)$ \\
$\mathrm{C}(3)-\mathrm{N}(2)-\mathrm{C}(7)-\mathrm{C}(6)$ & $1.0(4)$ \\
$\mathrm{Fe}(1)-\mathrm{N}(2)-\mathrm{C}(7)-\mathrm{C}(6)$ & $178.93(18)$ \\
$\mathrm{C}(3)-\mathrm{N}(2)-\mathrm{C}(7)-\mathrm{C}(8)$ & $179.9(2)$ \\
$\mathrm{Fe}(1)-\mathrm{N}(2)-\mathrm{C}(7)-\mathrm{C}(8)$ & $-2.1(3)$ \\
$\mathrm{C}(5)-\mathrm{C}(6)-\mathrm{C}(7)-\mathrm{N}(2)$ & $-0.3(4)$ \\
$\mathrm{C}(5)-\mathrm{C}(6)-\mathrm{C}(7)-\mathrm{C}(8)$ & $-179.1(2)$ \\
$\mathrm{C}(32)-\mathrm{N}(3)-\mathrm{C}(8)-\mathrm{C}(9)$ & $1.2(4)$ \\
$\mathrm{Fe}(1)-\mathrm{N}(3)-\mathrm{C}(8)-\mathrm{C}(9)$ & $179.6(2)$ \\
$\mathrm{C}(32)-\mathrm{N}(3)-\mathrm{C}(8)-\mathrm{C}(7)$ & $-178.0(2)$ \\
$\mathrm{Fe}(1)-\mathrm{N}(3)-\mathrm{C}(8)-\mathrm{C}(7)$ & $0.4(3)$ \\
$\mathrm{N}(2)-\mathrm{C}(7)-\mathrm{C}(8)-\mathrm{N}(3)$ & $1.1(3)$ \\
$\mathrm{C}(6)-\mathrm{C}(7)-\mathrm{C}(8)-\mathrm{N}(3)$ & $180.0(2)$ \\
$\mathrm{N}(2)-\mathrm{C}(7)-\mathrm{C}(8)-\mathrm{C}(9)$ & $-178.2(2)$ \\
$\mathrm{C}(6)-\mathrm{C}(7)-\mathrm{C}(8)-\mathrm{C}(9)$ & $0.6(4)$ \\
$\mathrm{C}(10)-\mathrm{C}(12)-\mathrm{C}(13)-\mathrm{C}(14)$ & $37.6(4)$ \\
$\mathrm{C}(11)-\mathrm{C}(12)-\mathrm{C}(13)-\mathrm{C}(14)$ & $-88.7(3)$ \\
$\mathrm{C}(10)-\mathrm{C}(12)-\mathrm{C}(13)-\mathrm{C}(18)$ & $-145.1(3)$ \\
$\mathrm{C}(11)-\mathrm{C}(12)-\mathrm{C}(13)-\mathrm{C}(18)$ & $88.6(3)$ \\
$\mathrm{C}(18)-\mathrm{C}(13)-\mathrm{C}(14)-\mathrm{C}(15)$ & $0.2(4)$ \\
$\mathrm{C}(12)-\mathrm{C}(13)-\mathrm{C}(14)-\mathrm{C}(15)$ & $177.6(3)$ \\
$\mathrm{C}(13)-\mathrm{C}(14)-\mathrm{C}(15)-\mathrm{C}(16)$ & $-1.3(4)$ \\
$\mathrm{C}(14)-\mathrm{C}(15)-\mathrm{C}(16)-\mathrm{C}(17)$ & $0.5(5)$ \\
$\mathrm{C}(15)-\mathrm{C}(16)-\mathrm{C}(17)-\mathrm{C}(18)$ & $1.4(4)$ \\
$\mathrm{C}(15)-\mathrm{C}(16)-\mathrm{C}(17)-\mathrm{C}(19)$ & $179.4(3)$ \\
$\mathrm{C}(14)-\mathrm{C}(13)-\mathrm{C}(18)-\mathrm{C}(17)$ & $1.8(4)$ \\
$\mathrm{C}(12)-\mathrm{C}(13)-\mathrm{C}(18)-\mathrm{C}(17)$ & $-175.6(2)$ \\
$\mathrm{C}(14)-\mathrm{C}(13)-\mathrm{C}(18)-\mathrm{N}(1)$ & $-171.0(2)$ \\
$\mathrm{C}(12)-\mathrm{C}(13)-\mathrm{C}(18)-\mathrm{N}(1)$ & $11.7(4)$ \\
& \\
& \\
&
\end{tabular}




\begin{tabular}{|c|c|}
\hline$C(16)-C(17)-C(18)-C(13)$ & $-2.6(4)$ \\
\hline $\mathrm{C}(19)-\mathrm{C}(17)-\mathrm{C}(18)-\mathrm{C}(13)$ & $179.5(3)$ \\
\hline $\mathrm{C}(16)-\mathrm{C}(17)-\mathrm{C}(18)-\mathrm{N}(1)$ & $170.0(2)$ \\
\hline $\mathrm{C}(19)-\mathrm{C}(17)-\mathrm{C}(18)-\mathrm{N}(1)$ & $-7.9(4)$ \\
\hline $\mathrm{C}(2)-\mathrm{N}(1)-\mathrm{C}(18)-\mathrm{C}(13)$ & $-104.3(3)$ \\
\hline $\mathrm{Fe}(1)-\mathrm{N}(1)-\mathrm{C}(18)-\mathrm{C}(13)$ & $77.5(3)$ \\
\hline $\mathrm{C}(2)-\mathrm{N}(1)-\mathrm{C}(18)-\mathrm{C}(17)$ & $82.7(3)$ \\
\hline $\mathrm{Fe}(1)-\mathrm{N}(1)-\mathrm{C}(18)-\mathrm{C}(17)$ & $-95.5(2)$ \\
\hline $\mathrm{C}(18)-\mathrm{C}(17)-\mathrm{C}(19)-\mathrm{C}(21)$ & $111.8(3)$ \\
\hline $\mathrm{C}(16)-\mathrm{C}(17)-\mathrm{C}(19)-\mathrm{C}(21)$ & $-66.0(3)$ \\
\hline $\mathrm{C}(18)-\mathrm{C}(17)-\mathrm{C}(19)-\mathrm{C}(20)$ & $-124.0(3)$ \\
\hline $\mathrm{C}(16)-\mathrm{C}(17)-\mathrm{C}(19)-\mathrm{C}(20)$ & $58.1(4)$ \\
\hline $\mathrm{Fe}(1)-\mathrm{O}(1)-\mathrm{C}(22)-\mathrm{O}(2)$ & $-7.5(3)$ \\
\hline $\mathrm{Fe}(1)-\mathrm{O}(1)-\mathrm{C}(22)-\mathrm{C}(23)$ & $169.0(3)$ \\
\hline $\mathrm{Fe}(1)-\mathrm{O}(2)-\mathrm{C}(22)-\mathrm{O}(1)$ & $7.7(3)$ \\
\hline $\mathrm{Fe}(1)-\mathrm{O}(2)-\mathrm{C}(22)-\mathrm{C}(23)$ & $-168.9(3)$ \\
\hline $\mathrm{C}(24)-\mathrm{C}(26)-\mathrm{C}(27)-\mathrm{C}(28)$ & $-58.0(4)$ \\
\hline $\mathrm{C}(25)-\mathrm{C}(26)-\mathrm{C}(27)-\mathrm{C}(28)$ & $65.7(4)$ \\
\hline $\mathrm{C}(24)-\mathrm{C}(26)-\mathrm{C}(27)-\mathrm{C}(32)$ & $123.5(3)$ \\
\hline $\mathrm{C}(25)-\mathrm{C}(26)-\mathrm{C}(27)-\mathrm{C}(32)$ & $-112.8(3)$ \\
\hline $\mathrm{C}(32)-\mathrm{C}(27)-\mathrm{C}(28)-\mathrm{C}(29)$ & $0.6(4)$ \\
\hline $\mathrm{C}(26)-\mathrm{C}(27)-\mathrm{C}(28)-\mathrm{C}(29)$ & $-178.0(3)$ \\
\hline $\mathrm{C}(27)-\mathrm{C}(28)-\mathrm{C}(29)-\mathrm{C}(30)$ & $-0.7(4)$ \\
\hline$C(28)-C(29)-C(30)-C(31)$ & $-1.0(4)$ \\
\hline $\mathrm{C}(29)-\mathrm{C}(30)-\mathrm{C}(31)-\mathrm{C}(32)$ & $2.6(4)$ \\
\hline $\mathrm{C}(29)-\mathrm{C}(30)-\mathrm{C}(31)-\mathrm{C}(33)$ & $-177.8(3)$ \\
\hline $\mathrm{C}(28)-\mathrm{C}(27)-\mathrm{C}(32)-\mathrm{C}(31)$ & $1.2(4)$ \\
\hline $\mathrm{C}(26)-\mathrm{C}(27)-\mathrm{C}(32)-\mathrm{C}(31)$ & $179.7(3)$ \\
\hline $\mathrm{C}(28)-\mathrm{C}(27)-\mathrm{C}(32)-\mathrm{N}(3)$ & $172.9(2)$ \\
\hline $\mathrm{C}(26)-\mathrm{C}(27)-\mathrm{C}(32)-\mathrm{N}(3)$ & $-8.6(4)$ \\
\hline $\mathrm{C}(30)-\mathrm{C}(31)-\mathrm{C}(32)-\mathrm{C}(27)$ & $-2.8(4)$ \\
\hline $\mathrm{C}(33)-\mathrm{C}(31)-\mathrm{C}(32)-\mathrm{C}(27)$ & $177.7(3)$ \\
\hline $\mathrm{C}(30)-\mathrm{C}(31)-\mathrm{C}(32)-\mathrm{N}(3)$ & $-174.6(2)$ \\
\hline $\mathrm{C}(33)-\mathrm{C}(31)-\mathrm{C}(32)-\mathrm{N}(3)$ & $5.9(4)$ \\
\hline $\mathrm{C}(8)-\mathrm{N}(3)-\mathrm{C}(32)-\mathrm{C}(27)$ & $88.5(3)$ \\
\hline $\mathrm{Fe}(1)-\mathrm{N}(3)-\mathrm{C}(32)-\mathrm{C}(27)$ & $-89.9(3)$ \\
\hline $\mathrm{C}(8)-\mathrm{N}(3)-\mathrm{C}(32)-\mathrm{C}(31)$ & $-99.5(3)$ \\
\hline $\mathrm{Fe}(1)-\mathrm{N}(3)-\mathrm{C}(32)-\mathrm{C}(31)$ & $82.1(3)$ \\
\hline $\mathrm{C}(30)-\mathrm{C}(31)-\mathrm{C}(33)-\mathrm{C}(35)$ & $-93.5(3)$ \\
\hline $\mathrm{C}(32)-\mathrm{C}(31)-\mathrm{C}(33)-\mathrm{C}(35)$ & $86.0(3)$ \\
\hline $\mathrm{C}(30)-\mathrm{C}(31)-\mathrm{C}(33)-\mathrm{C}(34)$ & $31.3(4)$ \\
\hline$C(32)-C(31)-C(33)-C(34)$ & $-149.2(3)$ \\
\hline $\mathrm{C}(48)-\mathrm{B}(1)-\mathrm{C}(36)-\mathrm{C}(37)$ & $-99.6(3)$ \\
\hline $\mathrm{C}(54)-\mathrm{B}(1)-\mathrm{C}(36)-\mathrm{C}(37)$ & $18.3(4)$ \\
\hline $\mathrm{C}(42)-\mathrm{B}(1)-\mathrm{C}(36)-\mathrm{C}(37)$ & $140.0(3)$ \\
\hline $\mathrm{C}(48)-\mathrm{B}(1)-\mathrm{C}(36)-\mathrm{C}(41)$ & $73.7(3)$ \\
\hline
\end{tabular}




\begin{tabular}{|c|c|}
\hline $\mathrm{C}(54)-\mathrm{B}(1)-\mathrm{C}(36)-\mathrm{C}(41)$ & $-168.4(2)$ \\
\hline $\mathrm{C}(42)-\mathrm{B}(1)-\mathrm{C}(36)-\mathrm{C}(41)$ & $-46.8(3)$ \\
\hline $\mathrm{C}(41)-\mathrm{C}(36)-\mathrm{C}(37)-\mathrm{C}(38)$ & $-0.6(4)$ \\
\hline $\mathrm{B}(1)-\mathrm{C}(36)-\mathrm{C}(37)-\mathrm{C}(38)$ & $172.9(3)$ \\
\hline $\mathrm{C}(36)-\mathrm{C}(37)-\mathrm{C}(38)-\mathrm{C}(39)$ & $1.0(4)$ \\
\hline $\mathrm{C}(37)-\mathrm{C}(38)-\mathrm{C}(39)-\mathrm{C}(40)$ & $-0.5(4)$ \\
\hline $\mathrm{C}(38)-\mathrm{C}(39)-\mathrm{C}(40)-\mathrm{C}(41)$ & $-0.3(4)$ \\
\hline$C(39)-C(40)-C(41)-C(36)$ & $0.8(5)$ \\
\hline$C(37)-C(36)-C(41)-C(40)$ & $-0.3(4)$ \\
\hline $\mathrm{B}(1)-\mathrm{C}(36)-\mathrm{C}(41)-\mathrm{C}(40)$ & $-174.3(3)$ \\
\hline $\mathrm{C}(36)-\mathrm{B}(1)-\mathrm{C}(42)-\mathrm{C}(47)$ & $124.0(3)$ \\
\hline $\mathrm{C}(48)-\mathrm{B}(1)-\mathrm{C}(42)-\mathrm{C}(47)$ & $6.5(4)$ \\
\hline $\mathrm{C}(54)-\mathrm{B}(1)-\mathrm{C}(42)-\mathrm{C}(47)$ & $-113.4(3)$ \\
\hline $\mathrm{C}(36)-\mathrm{B}(1)-\mathrm{C}(42)-\mathrm{C}(43)$ & $-52.1(3)$ \\
\hline $\mathrm{C}(48)-\mathrm{B}(1)-\mathrm{C}(42)-\mathrm{C}(43)$ & $-169.7(2)$ \\
\hline $\mathrm{C}(54)-\mathrm{B}(1)-\mathrm{C}(42)-\mathrm{C}(43)$ & $70.4(3)$ \\
\hline $\mathrm{C}(47)-\mathrm{C}(42)-\mathrm{C}(43)-\mathrm{C}(44)$ & $1.3(4)$ \\
\hline $\mathrm{B}(1)-\mathrm{C}(42)-\mathrm{C}(43)-\mathrm{C}(44)$ & $177.9(2)$ \\
\hline $\mathrm{C}(42)-\mathrm{C}(43)-\mathrm{C}(44)-\mathrm{C}(45)$ & $-0.5(4)$ \\
\hline $\mathrm{C}(43)-\mathrm{C}(44)-\mathrm{C}(45)-\mathrm{C}(46)$ & $-0.5(4)$ \\
\hline $\mathrm{C}(44)-\mathrm{C}(45)-\mathrm{C}(46)-\mathrm{C}(47)$ & $0.6(4)$ \\
\hline $\mathrm{C}(43)-\mathrm{C}(42)-\mathrm{C}(47)-\mathrm{C}(46)$ & $-1.3(4)$ \\
\hline $\mathrm{B}(1)-\mathrm{C}(42)-\mathrm{C}(47)-\mathrm{C}(46)$ & $-177.6(3)$ \\
\hline$C(45)-C(46)-C(47)-C(42)$ & $0.4(4)$ \\
\hline $\mathrm{C}(36)-\mathrm{B}(1)-\mathrm{C}(48)-\mathrm{C}(49)$ & $-176.8(2)$ \\
\hline $\mathrm{C}(54)-\mathrm{B}(1)-\mathrm{C}(48)-\mathrm{C}(49)$ & $62.3(3)$ \\
\hline $\mathrm{C}(42)-\mathrm{B}(1)-\mathrm{C}(48)-\mathrm{C}(49)$ & $-59.6(3)$ \\
\hline $\mathrm{C}(36)-\mathrm{B}(1)-\mathrm{C}(48)-\mathrm{C}(53)$ & $5.6(4)$ \\
\hline $\mathrm{C}(54)-\mathrm{B}(1)-\mathrm{C}(48)-\mathrm{C}(53)$ & $-115.4(3)$ \\
\hline $\mathrm{C}(42)-\mathrm{B}(1)-\mathrm{C}(48)-\mathrm{C}(53)$ & $122.7(3)$ \\
\hline $\mathrm{C}(53)-\mathrm{C}(48)-\mathrm{C}(49)-\mathrm{C}(50)$ & $-2.4(4)$ \\
\hline $\mathrm{B}(1)-\mathrm{C}(48)-\mathrm{C}(49)-\mathrm{C}(50)$ & $179.8(3)$ \\
\hline $\mathrm{C}(48)-\mathrm{C}(49)-\mathrm{C}(50)-\mathrm{C}(51)$ & $2.4(5)$ \\
\hline $\mathrm{C}(49)-\mathrm{C}(50)-\mathrm{C}(51)-\mathrm{C}(52)$ & $-0.7(5)$ \\
\hline$C(50)-C(51)-C(52)-C(53)$ & $-0.8(5)$ \\
\hline $\mathrm{C}(51)-\mathrm{C}(52)-\mathrm{C}(53)-\mathrm{C}(48)$ & $0.8(5)$ \\
\hline $\mathrm{C}(49)-\mathrm{C}(48)-\mathrm{C}(53)-\mathrm{C}(52)$ & $0.8(4)$ \\
\hline $\mathrm{B}(1)-\mathrm{C}(48)-\mathrm{C}(53)-\mathrm{C}(52)$ & $178.6(3)$ \\
\hline $\mathrm{C}(36)-\mathrm{B}(1)-\mathrm{C}(54)-\mathrm{C}(59)$ & $99.3(3)$ \\
\hline $\mathrm{C}(48)-\mathrm{B}(1)-\mathrm{C}(54)-\mathrm{C}(59)$ & $-142.8(3)$ \\
\hline $\mathrm{C}(42)-\mathrm{B}(1)-\mathrm{C}(54)-\mathrm{C}(59)$ & $-20.1(4)$ \\
\hline $\mathrm{C}(36)-\mathrm{B}(1)-\mathrm{C}(54)-\mathrm{C}(55)$ & $-82.4(3)$ \\
\hline $\mathrm{C}(48)-\mathrm{B}(1)-\mathrm{C}(54)-\mathrm{C}(55)$ & $35.5(3)$ \\
\hline $\mathrm{C}(42)-\mathrm{B}(1)-\mathrm{C}(54)-\mathrm{C}(55)$ & $158.2(2)$ \\
\hline$C(59)-C(54)-C(55)-C(56)$ & $1.7(4)$ \\
\hline $\mathrm{B}(1)-\mathrm{C}(54)-\mathrm{C}(55)-\mathrm{C}(56)$ & $-176.9(3)$ \\
\hline
\end{tabular}


$\mathrm{C}(54)-\mathrm{C}(55)-\mathrm{C}(56)-\mathrm{C}(57)$

$\mathrm{C}(55)-\mathrm{C}(56)-\mathrm{C}(57)-\mathrm{C}(58)$

$\mathrm{C}(56)-\mathrm{C}(57)-\mathrm{C}(58)-\mathrm{C}(59)$

$\mathrm{C}(57)-\mathrm{C}(58)-\mathrm{C}(59)-\mathrm{C}(54)$

$\mathrm{C}(55)-\mathrm{C}(54)-\mathrm{C}(59)-\mathrm{C}(58)$

$\mathrm{B}(1)-\mathrm{C}(54)-\mathrm{C}(59)-\mathrm{C}(58)$
$-0.8(4)$

$-0.5(4)$

$0.9(4)$

$0.0(4)$

$-1.3(4)$

177.2(3) 